\title{
Notes on Dumping Gold Beam in the AGS
}

\author{
C.J. Gardner, L.A. Ahrens, and P. Thieberger \\ Brookhaven National Laboratory, Upton, NY 11973, USA
}

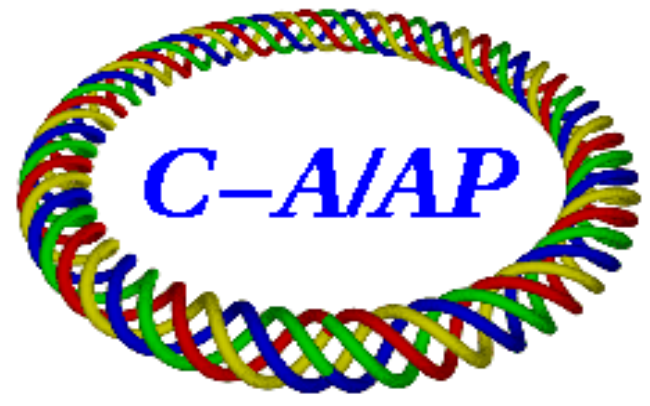

\section{Collider-Accelerator Department Brookhaven National Laboratory Upton, NY 11973}

Notice: This document has been authorized by employees of Brookhaven Science Associates, LLC under Contract No. DE-AC02-98CH10886 with the U.S. Department of Energy. The United States Government retains a nonexclusive, paid-up, irrevocable, world-wide license to publish or reproduce the published form of this document, or allow others to do so, for United States Government purposes. 


\title{
Notes on Dumping Gold Beam in the AGS
}

\author{
C.J. Gardner, L.A. Ahrens, and P. Thieberger
}

March 25, 2010

Localized losses of gold beam in the AGS during RHIC Run 8 produced vacuum leaks which required the replacement of several vacuum chambers. A review of what happened and why was given by Leif Ahrens at the Run 8 Retreat [1]. The following notes trace the subsequent development of clean dumping of gold beam on the beam dump in the J10 straight. The novel idea of stripping Au77+ ions in order to put them directly into the upstream face of the dump was introduced by Leif Ahrens and developed by all three of us. George Mahler made the actual stripping device and Dave Gassner developed its control. Leif Ahrens successfully commissioned the device with gold beam during Run 10 [2]. The reader may find it helpful to first view the figures herein and then refer to the text for details. Any errors in the notes are mine (Gardner) alone. 


\section{Contents}

1 The J10 Beam Dump 4

2 The Limiting Aperture 4

3 The Dump Bump 5

4 Putting Au77+ Ions into the Dump 6

5 Equations for Transport of Au79+ Ions $\quad 7$

6 Trajectories of Ions Stripped with a Plunging Stripper $\quad 9$

7 Separation between the Au79+ Trajectory and the Circu$\begin{array}{ll}\text { lating Beam } & 11\end{array}$

8 Trajectories of Ions Stripped with a Stationary Stripper 11

9 Trajectories of Ions Stripped at J10 12

10 Trajectories of Ions Stripped at J7 13

11 Trajectories of Ions Stripped at J5, J3, J1 14

12 Further Comments on Stripping at J7 15

13 Simulations of Where Ions Hit the Stripper 16

14 Energy Deposition in the Stripper 17

15 Temperature Rise with no Cooling $\quad 18$

16 Radiative Cooling during Energy Deposition 19

17 Radiative Cooling after Energy Deposition 21 
18 Heat Flow in the Foil

19 Solution of the Heat Flow Equations

20 Temperature at the Foil Edge

21 The Limit of very Small Hit Width

22 The Case of Heat Flowing into the Foil Edge

23 Inclined Plunging Stripper

25 Observed Loss Patterns with and without the Plunging Stripper.

26 Energy Deposition in the J10 Dump 


\section{The J10 Beam Dump}

The J10 beam dump is documented in several AGS Department drawings. Its location in the J10 straight is shown schematically in Figure 1. (This is a reproduction of Figure 3 of Ref. [3] with the addition of the dump mass.) Here

$$
\begin{aligned}
L_{1} & =22.12367 \text { inches } \\
L_{d} & =79.62 \text { inches } \\
L_{2} & =18.24367 \text { inches } \\
T & =2 \times 59.99367 \text { inches }
\end{aligned}
$$

and

$$
L_{1}+L_{d}+L_{2}=T .
$$

The lengths $L_{1}$ and $L_{d}$ are derived from drawings D08-M-216 and D08-M-227. The length $T$ is the drift length in the J10 straight. This is given by Eq. (183) of Ref. [3].

Drawings D08-M-208, 209, 211, 212, 213 and 214 show details of the dump mass. The part of the mass that is exposed to beam is made of copper and is referred to as the "copper block" in the drawings. The copper is water cooled and is surrounded by stainless steel.

\section{The Limiting Aperture}

The horizontal and vertical apertures in the AGS are given in Ref. [4]. Let $X(s)$ be the horizontal half-aperture at longitudinal position $s$ along the design orbit in AGS, and let $\beta_{x}(s)$ be the beta function there. We define

$$
E_{x}(s)=\frac{X^{2}(s)}{\beta_{x}(s)}
$$

and denote by $e_{x}$ the minimum value attained by $E_{x}(s)$. Then

$$
e_{x}=\frac{X^{2}\left(s_{0}\right)}{\beta_{x}\left(s_{0}\right)}
$$

where $s_{0}$ is the position where $E_{x}$ attains its minimum. We call $e_{x}$ the

horizontal acceptance of the machine. The limiting aperture is

$$
X_{L}=X\left(s_{0}\right)
$$


and the horizontal acceptance envelope is given by

$$
H(s)=\sqrt{e_{x} \beta_{x}(s)} .
$$

The limiting aperture in AGS occurs at the injection kicker in the A5 straight. Here $X=2.531$ inches [5]. Table 1 lists $\beta_{x}$ at the kicker and the resulting acceptance $e_{x}$ for various values of the horizontal and vertical tunes $Q_{H}$ and $Q_{V}$. The $H(u)$ and $H(d)$ columns give the acceptance envelope at the upstream and downstream ends of the J10 dump respectively.

Table 1: AGS Horizontal Acceptance. The units of $\beta_{x}$ and $e_{x}$ are meters and $\mathrm{mm}$ mrad respectively; those of $H(u)$ and $H(d)$ are inches.

\begin{tabular}{|c|c|c|c|c|c|}
\hline$Q_{H}$ & $Q_{V}$ & $\beta_{x}$ & $e_{x}$ & $H(u)$ & $H(d)$ \\
\hline 8.83 & 8.75 & 24.3749 & 169.6884 & 2.2244 & 1.8500 \\
8.79 & 8.75 & 23.8028 & 173.7668 & 2.2334 & 1.8650 \\
8.75 & 8.75 & 23.2435 & 177.9479 & 2.2441 & 1.8817 \\
8.75 & 8.79 & 23.7258 & 174.3307 & 2.2298 & 1.8635 \\
8.75 & 8.83 & 24.2129 & 170.8239 & 2.2172 & 1.8469 \\
\hline 8.70 & 8.75 & 22.5619 & 183.3241 & 2.2599 & 1.9052 \\
8.70 & 8.70 & 21.9710 & 188.2543 & 2.2838 & 1.9337 \\
8.75 & 8.70 & 22.6478 & 182.6288 & 2.2643 & 1.9068 \\
\hline 8.70 & 8.90 & 24.3805 & 169.6490 & 2.2052 & 1.8356 \\
\hline
\end{tabular}

The $H(u)$ numbers show that if the dump mass is positioned so that its upstream end is less than 2.2052 inches from the OCO then it becomes the limiting aperture for any of the tunes listed in the table. (The OCO is the optimum closed orbit shown schematically in Figure 1.) In practice the downstream end of the dump mass is moved far enough away from the OCO to ensure that beam hits the upstream end first.

\section{The Dump Bump}

The dump bump is a three-halves lambda distortion of the closed orbit in the region of the J10 dump. It is produced by backleg windings on magnets I10, I11, J4, J5, J18, J19, K12 and K13. The I10, I11, J19, and K12 magnets are "short" magnets with an iron length of 75 inches. The 
J4, J5, J18, and K13 magnets are "long" magnets with an iron length of 90 inches. The backleg windings on the short and long magnets consist of 6 and 5 turns respectively so that a given current produces the same beam deflection in each magnet. The windings on all 8 magnets are connected together in a series string with the I10, I11, K12, and K13 windings having one polarity and the J4, J5, J18, and J19 windings having the opposite polarity. This arrangement produces the desired orbit distortion at the dump and ensures that no net EMF is induced in the string by the main windings. The series string is connected to a monopolar power supply capable of delivering up to 700 Amps.

The dump bump is shown in Figure 2. Here the black curve is the bump and the blue lines show the outline of the dump. Outside the bump region there is a residual orbit distortion that depends on the horizontal tune. Here the horizontal and vertical tunes are $Q_{H}=8.75$ and $Q_{V}=8.80$.

\section{Putting Au77+ Ions into the Dump}

Figures 3 through 7 illustrate the "standard" way of putting gold ions $(\mathrm{Au} 77+)$ into the dump.

In Figure 3 the black curve is the dump bump and the blue lines show the outline of the dump. The violet curves show the envelope of a $0.9 \pi$ emittance beam. This is the physical size of gold beam in AGS at extraction assuming a normalized emittance of $10 \pi$. Beam is put into the dump by increasing the amplitude of the bump. The downstream end of the dump may be moved further to the inside (more negative) than shown to ensure that beam hits the upstream end. The circulating beam direction here is from left to right.

In Figure 4 the bump amplitude has been inreased causing beam loss on the dump and reducing the beam emittance to $0.55 \pi$.

In Figure 5 the bump amplitude has been inreased further, again causing beam loss on the dump and reducing the beam emittance to $0.3 \pi$.

In Figure 6 the bump amplitude has been inreased further still, causing beam loss on the dump and reducing the beam emittance to $0.1 \pi$.

In Figure 7 the bump amplitude has been inreased further, causing beam loss on the dump and reducing the beam emittance to 0 .

Experience has shown that this method of putting gold ions into the dump 
produces unwanted losses downstream. This is due to scattering at the surface of the dump as illustrated by the blue and red arrows in Figure 8 . It is believed that some of the scattered particles are Au79+ ions. Because the rigidity is reduced by the factor $77 / 79$, the Au79+ ion is no longer inside the machine acceptance and is eventually lost on an aperture. This will be illustrated in Section 9 .

In order to eliminate scattering at the dump surface, Leif Ahrens proposed stripping the Au77+ ions at a point upstream so that the resulting Au79+ ions are put directly into the upstream face of the dump. This is

illustrated by the green and purple arrows in Figure 8. Here the scattered particles are absorbed by the dump.

\section{Equations for Transport of Au79+ Ions}

So, let us consider a Au77+ ion that has been stripped of its two electrons at some point in the machine acceptance. Let $X_{0}$ and $X_{0}^{\prime}$ be the position and angle (with respect to the design orbit) of the ion at this point and let $X$ and $X^{\prime}$ be the position and angle at some point downstream. Let $s_{0}$ and $s$ be the coordinates of the corresponding points on the design orbit. As shown in the Appendix we have

$$
\mathbf{X}=\mathbf{M} \mathbf{X}_{0}+\frac{\Delta p}{p}\left(\mathbf{D}-\mathbf{M D}_{0}\right)+\left(1-\frac{\Delta p}{p}\right)\left(\mathbf{d}-\mathbf{M d}_{0}\right)
$$

or equivalently

$$
\mathbf{X}-\frac{\Delta p}{p} \mathbf{D}-\left(1-\frac{\Delta p}{p}\right) \mathbf{d}=\mathbf{M}\left\{\mathbf{X}_{0}-\frac{\Delta p}{p} \mathbf{D}_{0}-\left(1-\frac{\Delta p}{p}\right) \mathbf{d}_{0}\right\}
$$

where

$$
\begin{array}{cc}
\mathbf{X}=\left(\begin{array}{c}
X \\
X^{\prime}
\end{array}\right), & \mathbf{X}_{0}=\left(\begin{array}{c}
X_{0} \\
X_{0}^{\prime}
\end{array}\right) \\
\mathbf{D}=\left(\begin{array}{c}
D \\
D^{\prime}
\end{array}\right), & \mathbf{D}_{0}=\left(\begin{array}{c}
D_{0} \\
D_{0}^{\prime}
\end{array}\right) \\
\mathbf{d}=\left(\begin{array}{c}
d \\
d^{\prime}
\end{array}\right), & \mathbf{d}_{0}=\left(\begin{array}{c}
d_{0} \\
d_{0}^{\prime}
\end{array}\right)
\end{array}
$$

and

$$
\mathbf{M}=\left(\begin{array}{ll}
M_{11} & M_{12} \\
M_{21} & M_{22}
\end{array}\right)
$$


Here $D_{0}, D_{0}^{\prime}$ and $D, D^{\prime}$ are the periodic dispersion functions at $s_{0}$ and $s$ respectively. $d_{0}, d_{0}^{\prime}$ and $d, d^{\prime}$ are the closed orbit positions and angles at $s_{0}$ and $s$ respectively. The elements of $\mathbf{M}$ are

$$
\begin{gathered}
M_{11}=\sqrt{\beta / \beta_{0}}\left(C+\alpha_{0} S\right), \quad M_{12}=\sqrt{\beta \beta_{0}} S \\
M_{21}=-\left(\frac{\alpha-\alpha_{0}}{\sqrt{\beta \beta_{0}}}\right) C-\left(\frac{1+\alpha \alpha_{0}}{\sqrt{\beta \beta_{0}}}\right) S, \quad M_{22}=\sqrt{\beta_{0} / \beta}(C-\alpha S)
\end{gathered}
$$

where $\alpha_{0}, \beta_{0}$ and $\alpha, \beta$ are the Courant-Snyder parameters at $s_{0}$ and $s$ respectively. The parameters $C$ and $S$ are

$$
C=\cos \mu, \quad S=\sin \mu
$$

where $\mu$ is the betatron phase advance between $s_{0}$ and $s$.

The stripping of Au77+ to Au79+ is taken into account by setting

$$
\frac{\Delta p}{p}=-\frac{2}{77}
$$

in (10) and (11).

Writing out the components of (10) we have

$$
\begin{aligned}
X & =M_{11} X_{0}+M_{12} X_{0}^{\prime} \\
& +\frac{\Delta p}{p}\left(D-M_{11} D_{0}-M_{12} D_{0}^{\prime}\right) \\
& +\left(1-\frac{\Delta p}{p}\right)\left(d-M_{11} d_{0}-M_{12} d_{0}^{\prime}\right) \\
X^{\prime} & =M_{21} X_{0}+M_{22} X_{0}^{\prime} \\
& +\frac{\Delta p}{p}\left(D^{\prime}-M_{21} D_{0}-M_{22} D_{0}^{\prime}\right) \\
& +\left(1-\frac{\Delta p}{p}\right)\left(d^{\prime}-M_{21} d_{0}-M_{22} d_{0}^{\prime}\right)
\end{aligned}
$$

and defining

$$
\begin{gathered}
\Delta D=D-M_{11} D_{0}-M_{12} D_{0}^{\prime} \\
\Delta d=d-M_{11} d_{0}-M_{12} d_{0}^{\prime} \\
\mathcal{X}=M_{11} X_{0}+M_{12} X_{0}^{\prime}+\Delta d
\end{gathered}
$$




$$
\begin{gathered}
\Delta D^{\prime}=D^{\prime}-M_{21} D_{0}-M_{22} D_{0}^{\prime} \\
\Delta d^{\prime}=d^{\prime}-M_{21} d_{0}-M_{22} d_{0}^{\prime} \\
\mathcal{X}^{\prime}=M_{21} X_{0}+M_{22} X_{0}^{\prime}+\Delta d^{\prime}
\end{gathered}
$$

we have

$$
\begin{gathered}
X=\mathcal{X}+\frac{\Delta p}{p}(\Delta D-\Delta d) \\
X^{\prime}=\mathcal{X}^{\prime}+\frac{\Delta p}{p}\left(\Delta D^{\prime}-\Delta d^{\prime}\right) .
\end{gathered}
$$

Here $\mathcal{X}$ and $\mathcal{X}^{\prime}$ give the trajectory that a Au77+ ion would have if it were not stripped at $s_{0}$. Note that if there are no perturbing dipoles between $s_{0}$ and $s$ then $\Delta d=0$ and $\Delta d^{\prime}=0$. Similarly, if there are no dispersive elements between $s_{0}$ and $s$ then $\Delta D=0$ and $\Delta D^{\prime}=0$.

Note also that if the phase advance between $s_{0}$ and $s$ is a multiple of $\pi$ then the $M_{12}$ matrix element is zero and it follows that $\mathcal{X}$ and $X$ are independent of $X_{0}^{\prime}$. This means that Au79+ ions created by stripping at a given point on the dump surface will all have the same coordinate $X$ at locations downstream that are a multiple of $\pi$ in phase advance away from the dump. If these locations also happen to be at horizontal beta maximums then one can have localized losses of Au79+ ions.

\section{Trajectories of Ions Stripped with a Plunging Stripper}

Au77+ ions can be stripped either by plunging a stripper into the circulating beam or by moving the circulating beam onto a stationary stripper. Here we consider the former case which was proposed by Ahrens.

For any $Y_{0}$ and $Y_{0}^{\prime}$ we have

$$
\begin{gathered}
M_{11} Y_{0}+M_{12} Y_{0}^{\prime}=\sqrt{\beta / \beta_{0}}\left\{C Y_{0}+S\left(\alpha_{0} Y_{0}+\beta_{0} Y_{0}^{\prime}\right)\right\} \\
M_{21} Y_{0}+M_{22} Y_{0}^{\prime}=\frac{1}{\sqrt{\beta \beta_{0}}}\left\{(C-\alpha S)\left(\alpha_{0} Y_{0}+\beta_{0} Y_{0}^{\prime}\right)-(S+\alpha C) Y_{0}\right\}
\end{gathered}
$$

and it follows that

$$
\Delta D=D-\sqrt{\beta / \beta_{0}}\left\{C D_{0}+S\left(\alpha_{0} D_{0}+\beta_{0} D_{0}^{\prime}\right)\right\}
$$




$$
\begin{gathered}
\Delta d=d-\sqrt{\beta / \beta_{0}}\left\{C d_{0}+S\left(\alpha_{0} d_{0}+\beta_{0} d_{0}^{\prime}\right)\right\} \\
\mathcal{X}=\sqrt{\beta / \beta_{0}}\left\{C X_{0}+S\left(\alpha_{0} X_{0}+\beta_{0} X_{0}^{\prime}\right)\right\}+\Delta d
\end{gathered}
$$

and

$$
\begin{array}{r}
\Delta D^{\prime}=D^{\prime}-\frac{1}{\sqrt{\beta \beta_{0}}}\left\{(C-\alpha S)\left(\alpha_{0} D_{0}+\beta_{0} D_{0}^{\prime}\right)-(S+\alpha C) D_{0}\right\} \\
\Delta d^{\prime}=d^{\prime}-\frac{1}{\sqrt{\beta \beta_{0}}}\left\{(C-\alpha S)\left(\alpha_{0} d_{0}+\beta_{0} d_{0}^{\prime}\right)-(S+\alpha C) d_{0}\right\} \\
\mathcal{X}^{\prime}=\frac{1}{\sqrt{\beta \beta_{0}}}\left\{(C-\alpha S)\left(\alpha_{0} X_{0}+\beta_{0} X_{0}^{\prime}\right)-(S+\alpha C) X_{0}\right\}+\Delta d^{\prime} .
\end{array}
$$

Now since a stripper moving into the circulating beam at $s_{0}$ strips the maximum amplitude ions, we have

$$
\begin{gathered}
\alpha_{0}\left(X_{0}-d_{0}\right)+\beta_{0}\left(X_{0}^{\prime}-d_{0}^{\prime}\right)=0 \\
\alpha_{0} X_{0}+\beta_{0} X_{0}^{\prime}=\alpha_{0} d_{0}+\beta_{0} d_{0}^{\prime}
\end{gathered}
$$

and it follows from the equations above that

$$
\begin{gathered}
\mathcal{X}=d+\sqrt{\beta / \beta_{0}} C\left(X_{0}-d_{0}\right) \\
\mathcal{X}^{\prime}=d^{\prime}-\frac{1}{\sqrt{\beta \beta_{0}}}(S+\alpha C)\left(X_{0}-d_{0}\right) .
\end{gathered}
$$

We shall assume that the stripper is plunged from the inside side of the ring. Then

$$
X_{0}=d_{0}-\sqrt{\epsilon \beta_{0}}
$$

where $\pi \epsilon$ is the emittance of the circulating beam. Using this in (40) and (41), and recalling (28) and (29) we obtain

$$
\begin{gathered}
X=d-C \sqrt{\epsilon \beta}+\frac{\Delta p}{p}(\Delta D-\Delta d) \\
X^{\prime}=d^{\prime}+\sqrt{\epsilon / \beta}(S+\alpha C)+\frac{\Delta p}{p}\left(\Delta D^{\prime}-\Delta d^{\prime}\right) .
\end{gathered}
$$

Here we see that the positions of stripped ions at $s$ will cover a range of width $C \sqrt{\epsilon \beta}$ as the stripper at $s_{0}$ is plunged into the beam. In order to deposit the stripped ions on a dump at $s$ with localized heating kept to a minimum, we must therefore keep the phase advance between $s_{0}$ and $s$ away from $\pi / 2$. In Section 10 we will see that this can be achieved by putting the stripper in the $\mathrm{J} 7$ straight. 


\section{Separation between the Au79+ Trajectory and the Circulating Beam}

The inside edge of the circulating beam envelope at $s$ is

$$
X_{e}=d-\sqrt{\epsilon \beta}
$$

and the separation between the Au79+ trajectory and the envelope at this point is

$$
X_{e}-X=-(1-C) \sqrt{\epsilon \beta}-\frac{\Delta p}{p}(\Delta D-\Delta d)
$$

where

$$
\begin{gathered}
\Delta D=D-\sqrt{\beta / \beta_{0}}\left\{C D_{0}+S\left(\alpha_{0} D_{0}+\beta_{0} D_{0}^{\prime}\right)\right\} \\
\Delta d=d-\sqrt{\beta / \beta_{0}}\left\{C d_{0}+S\left(\alpha_{0} d_{0}+\beta_{0} d_{0}^{\prime}\right)\right\} .
\end{gathered}
$$

In Section 10 we will see that a separation of some $7 \mathrm{~mm}$ at the J10 dump is obtained with a stripper in the J7 straight.

\section{Trajectories of Ions Stripped with a Stationary Stripper}

Let us now consider the case in which the stripper is held fixed and a programmable bump is used to move the circulating beam onto the

stripper. We shall assume that the stripper edge is to the outside of the closed orbit at $s_{0}$. We then have

$$
X_{0}=d_{0}+\sqrt{\epsilon \beta}
$$

where $X_{0}$ is fixed and

$$
\alpha_{0} X_{0}+\beta_{0} X_{0}^{\prime}=\alpha_{0} d_{0}+\beta_{0} d_{0}^{\prime}
$$

Beam is moved onto the stripper by increasing $d_{0}$. Using (49) in (40) and (41), and recalling (28) and (29) we obtain

$$
\begin{gathered}
X=d+C \sqrt{\epsilon \beta}+\frac{\Delta p}{p}(\Delta D-\Delta d) \\
X^{\prime}=d^{\prime}-\sqrt{\epsilon / \beta}(S+\alpha C)+\frac{\Delta p}{p}\left(\Delta D^{\prime}-\Delta d^{\prime}\right)
\end{gathered}
$$


where

$$
\begin{gathered}
\Delta D=D-\sqrt{\beta / \beta_{0}}\left\{C D_{0}+S\left(\alpha_{0} D_{0}+\beta_{0} D_{0}^{\prime}\right)\right\} \\
\Delta d=d-\sqrt{\beta / \beta_{0}}\left\{C d_{0}+S\left(\alpha_{0} d_{0}+\beta_{0} d_{0}^{\prime}\right)\right\} .
\end{gathered}
$$

For the special case in which the phase advance between $s_{0}$ and $s$ is $\pi / 2$ we have

$$
C=0, \quad S=1
$$

and

$$
X=d+\frac{\Delta p}{p}(\Delta D-\Delta d)
$$

where

$$
\begin{gathered}
\Delta D=D-\sqrt{\beta / \beta_{0}}\left(\alpha_{0} D_{0}+\beta_{0} D_{0}^{\prime}\right) \\
\Delta d=d-\sqrt{\beta / \beta_{0}}\left(\alpha_{0} d_{0}+\beta_{0} d_{0}^{\prime}\right) .
\end{gathered}
$$

The phase advance between the J1 straight and the J10 dump is in fact very close to $\pi / 2$. In Section 11 we will see that $X=-58 \mathrm{~mm}$ can be obtained at the J10 dump with a fixed stripper in the J1 straight.

Furthermore the closed orbit can be manipulated in a way that produces a spread of positions $X$ at the dump.

\section{Trajectories of Ions Stripped at J10}

Figures 9 through 11 illustrate the case in which a Au77+ ion is stripped to Au79+ by just grazing the surface of the J10 dump. The red curve in Figure 9 is the trajectory of such an ion. The green curve is the trajectory of an unstripped Au77+ ion with the same initial conditions. The black curve is the dump bump. The violet curves show the envelope of the $0.9 \pi$ emittance beam and the blue lines show the outline of the dump.

Figure 10 is a "zoomed out" view of Figure 9 showing the trajectory of the stripped Au79+ ion. Going from left to right, the local minima of the trajectory are located in the $\mathrm{J} 13, \mathrm{~J} 17, \mathrm{~K} 1$, and $\mathrm{K} 5$ straights respectively. During Run 8 high loss was observed at J17 before the dump position was optimized and before the dump bump was strengthened.

In Figure 11 we have "zoomed out" further. The solid brown curve is the equilibrium orbit about which the Au79+ ion is oscillating. The dashed curve is the periodic dispersion multiplied by $\Delta p / p=-2 / 77$. The minimum of the Au79+ trajectory near $s=782 \mathrm{~m}$ is in the L13 straight. 
During Run 10 beam loss was observed at L9 when unstripped beam $(\mathrm{Au} 77+)$ was put into the dump.

In Figure 12 the red curve is the trajectory of a gold ion that has been stripped to Au78+ by just grazing the surface of the dump. The solid brown curve is the equilibrium orbit about which the Au78+ ion is oscillating. The dashed curve is the periodic dispersion multiplied by $\Delta p / p=-1 / 77$. The minimum of the Au78+ trajectory near $s=782 \mathrm{~m}$ is in the L13 straight.

\section{Trajectories of Ions Stripped at J7}

Figures 13 through 19 illustrate the process of dumping beam on the J10 dump by plunging a stripper into the beam in the $\mathrm{J} 7$ straight.

In Figure 13 the red curve shows the trajectory of a gold ion that has been stripped to Au79+ by passing through a stripper located in the J7 straight. The green curve is the trajectory of an unstripped Au77+ ion with the same initial coordinates. As before, the black curve is the dump bump, the violet curves show the envelope of the $0.9 \pi$ emittance beam, and the blue lines show the outline of the dump. The stripped ion is lost on the upstream face of the dump.

In Figure 14 the J7 stripper has been plunged further into the beam (keeping the dump bump fixed) thereby reducing the circulating beam emittance while putting the stripped beam into the upstream face of the dump. The process continues in Figures 15, 16, and 17 with the end result that all of the circulating beam is deposited into face of the dump.

Figure 18 shows the initial and final Au79+ ion trajectories and Figure 19 gives a "zoomed out" view of the same.

These figures show that the range of trajectory positions at the face of the dump is some $4 \mathrm{~mm}$ wide. Note that the magnet vacuum chamber wall is at -3.406 inches $(-86.5 \mathrm{~mm})$, well away from the trajectories. The figures also show that if the stripper should fail to plunge, the circulating Au77+ beam still can be put into the dump by increasing the dump bump amplitude just a small amount. Thus, if the dump bump is programed to always increase in amplitude just after the plunging time, one is assured that the beam will be put into the dump one way or the other. 


\section{Trajectories of Ions Stripped at J5, J3, J1}

Figure 20 shows the initial and final Au79+ trajectories obtained for the case in which a stripper is plunged into the circulating beam in the $\mathrm{J} 5$ straight. Note that in this case the dump bump has to move a greater distance in order to put the circulating Au77+ beam into the dump should the stripper fail to plunge.

Figure 21 shows the initial and final Au79+ trajectories for the case in which a stripper is plunged in the J3 straight. Note that because the phase advance between $\mathrm{J} 3$ and $\mathrm{J} 10$ is close to $\pi / 2$, the trajectories converge to a point on the upstream face of the dump. This could produce undesirable localized heating.

Figure 22 shows the initial and final Au79+ trajectories for the case in which a stripper is plunged (now from the outside) in the J1 straight. The phase advance between $\mathrm{J} 1$ and $\mathrm{J} 10$ is still close to $\pi / 2$, so the trajectories are again close to one another on the upstream face of the dump.

In Figure 23 the stripper is held fixed in the J1 straight while the circulating beam is moved into it with a programmable bump. Figure $\mathbf{2 4}$ is a "zoomed in" view of Figure 23.

Figure 25 shows the closed orbit and Au79+ trajectory of Figure 24 and the Au79+ trajectory obtained with the closed orbit moved just to the point where all circulating beam has been removed. Here we see that a range of trajectory positions some $6 \mathrm{~mm}$ wide is obtained at the face of the dump. In order to obtain these trajectories the position and angle of the closed orbit at the J1 stripper need to be programmed. This could be done with a three-halves lambda position bump and a lambda angle bump. The position bump would be produced by backleg windings on magnets I1, I2, I15, I16, J9, J10, K3, and K4 where "long" magnets I15, I16, K3, and K4 would have 5 turns and "short" magnets I1, I2, J9, and J10 would have 6 turns. The angle bump would be produced by the backleg windings on magnets I8, I9, J16, and J17 where long magnets I8, J16, and J17 would have 5 turns and short magnet I9 would have 6 turns. For the position bump the windings on all 8 magnets would be connected together in a series string with the I1, I2, K3, and K4 windings having one polarity and the I15, I16, J9, and J10 windings having the opposite polarity. For the angle bump the windings on all 4 magnets would be connected together in a series string with the I8 and I9 windings having one polarity and the J16 and J17 windings having the opposite polarity. Figure 26 shows the J1 
position and angle bumps (orange and brown curves) and their superposition (black curve).

\section{Further Comments on Stripping at J7}

Figure 27 shows the Au79+ trajectories (red curves) resulting from plunging the J7 stripper from the inside and from the outside. As before, the black curve is the dump bump and the violet curves show the envelope of the nominal $0.9 \pi \mathrm{mm}$ mrad emittance circulating beam. In the plunging-from-inside case, the Au79+ trajectories hit the upstream face of the dump. In the plunging-from-outside case, the Au79+ trajectories still hit the dump but they miss the upstream face. This may result in a dirtier dump with more debris downstream.

In Figure 28 we see that we can make the plunging-from-outside trajectories hit the upstream face of the dump, but it requires bringing the circulating beam very close to the dump. This may make it difficult to set up the stripping in practice.

Figure 29 shows stripping from the inside at $\mathrm{J} 7$ with twice the nominal circulating beam emittance $(2 \times 0.9 \pi=1.8 \pi \mathrm{mm} \mathrm{mrad})$. Here we see that there is still clearance between the dump and the circulating beam.

Figure 30 shows stripping of Au77+ to Au79+ and Au78+ in the J7 straight. The solid and dashed red curves are the Au79+ and Au78+ trajectories respectively. The circulating beam emittance is $0.9 \pi \mathrm{mm} \mathrm{mrad}$.

Figure 31 is a "zoomed out" view of Figure 30 showing the Au78+ trajectory that survives beyond the J10 dump. The minima of trajectory near 647 and $768 \mathrm{~m}$ are in the J13 and L9 straights respectively.

In Figure 32 we again have stripping from Au77+ to Au79+ and Au78+ in the J7 straight, but now with the dump bump adjusted so that both the Au79+ and the Au78+ ions hit the dump. The solid and dashed red curves are the Au79+ and Au78+ trajectories respectively. Note that there is still clearance between the dump and the circulating beam. The circulating beam emittance is $0.9 \pi \mathrm{mm}$ mrad. 


\section{Simulations of Where Ions Hit the Stripper}

As the stripper is plunged into the circulating beam, ions pass through the stripper material depositing some of their energy as heat. In order to determine how hot the material gets we need to know the dimensions of the region where ions hit the material surface. This is illustrated in

Figure 33. Here $w$ is the maximum distance of ion hits from the stripper edge and $h$ is the smallest distance along the edge that contains the hits. The hit area is approximately equal to $w h$. The maximum hit distance is sensitive to the horizontal tune. Figures $\mathbf{3 4}$ and $\mathbf{3 5}$ show the result of a simulation in which a stripper is plunged into circulating beam and the maximum hit distance is recorded as a function of tune. The distance is given in units of beam half-width $\sqrt{\epsilon \beta_{0}}$ where $\pi \epsilon$ is the beam emittance and $\beta_{0}$ is the horizontal beta function at the stripper. The beam distribution is assumed to be uniform in $X, X^{\prime}$ space. The stripper is plunged a distance of $\sqrt{\epsilon \beta_{0}}$ into the beam in steps of $0.002 \sqrt{\epsilon \beta_{0}}$ per turn. In Figure 35 the red, blue, and green curves give the maximum hit distances for plunging steps of $0.0015,0.001$, and $0.0005 \sqrt{\epsilon \beta_{0}}$ per turn.

Figures 36 and $\mathbf{3 7}$ show the distribution of hits on the stripper for various tunes. Here the red histogram was obtained with horizontal tune $Q_{H}=0.286$; the blue with $Q_{H}=0.281$; and the green with $Q_{H}=0.250$. These are again the results of simulations in which a stripper is plunged into circulating beam and the distance of ion hits from the stripper edge is recorded. The beam distribution is assumed to be uniform in $X, X^{\prime}$ space and the stripper is plunged a distance of $\sqrt{\epsilon \beta_{0}}$ into the beam in steps of $0.002 \sqrt{\epsilon \beta_{0}}$ per turn. The beam half-width is taken to be $\sqrt{\epsilon \beta_{0}}=2.7 \mathrm{~mm}$. Note that the revolution period of gold ions at AGS extraction is $2.7 \mu \mathrm{s}$. This gives a plunging speed of $2.0 \mathrm{~m} / \mathrm{s}$ which is the maximum capability of the plunging mechanism.

Figures 38 through $\mathbf{4 5}$ are a sequence of figures showing the order in which particles of the initial beam distribution hit the stripper as it is plunged into the beam. The stripper step per turn is 0.002 of the beam half-width and 500 steps are required to strip all particles in the distribution. The horizontal tune is $Q_{H}=0.281$. 


\section{Energy Deposition in the Stripper}

The material chosen for the stripper is tungsten foil. We have the following numbers:

1. The tungsten foil is $d=0.001$ inches thick.

2. The density of tungsten is $\rho=19.3 \mathrm{~g} / \mathrm{cm}^{3}[6]$.

3. This gives surface density $\rho d=49.022 \mathrm{mg} / \mathrm{cm}^{2}$.

4. The kinetic energy of a gold ion at AGS extraction is $8.86 \mathrm{GeV}$ per nucleon.

5. The rate of energy loss of a fully stripped gold ion traveling through tungsten at this energy is

$$
-\frac{d E}{d x}=8 \mathrm{MeV} \mathrm{cm}^{2} / \mathrm{mg} .
$$

This number comes from Peter Thieberger's plot of $d E / d x$ versus $E$ obtained using Ziegler's computer code [7].

6. The energy deposited by a single gold ion traversing the foil at AGS extraction energy is then

$$
\mathcal{E}=-\frac{d E}{d x} \rho d=392 \mathrm{MeV}
$$

which gives (using $1 \mathrm{eV}=1.602176462(63) \times 10^{-19}$ Joules)

$$
\mathcal{E}=6.28 \times 10^{-11} \text { Joules. }
$$

7. A single bunch at AGS extraction can have as many as

$$
N=1.5 \times 10^{9}
$$

gold ions.

8. A single bunch traversing the foil deposits energy

$$
N \mathcal{E}=0.09425 \text { Joules. }
$$


9. Each AGS cycle 4 bunches pass through the foil. This gives a total energy deposition per AGS cycle of

$$
4 N \mathcal{E}=0.377 \text { Joules }
$$

10. We assume that the beam half-width at the stripper foil is $2.7 \mathrm{~mm}$ and that the foil is plunged into the beam at $2.0 \mathrm{~m} / \mathrm{s}$.

11. This gives energy deposition time

$$
\tau=1.35 \mathrm{~ms}
$$

\section{Temperature Rise with no Cooling}

We have the following numbers:

1. The specific heat of tungsten is $[6]$

$$
c=0.132 \mathrm{~J} /(\mathrm{gK}) .
$$

2. The melting point is $3414 \mathrm{C}=3687.15 \mathrm{~K}[6]$.

Let us assume that the area of foil exposed to beam is

$$
A=2.0 \mathrm{~mm}^{2} .
$$

The mass of tungsten exposed to beam is then

$$
\rho d A=0.9804 \mathrm{mg}
$$

and, if there is no heat flow from the exposed part of the foil, the rise in temperature due to 4 bunches passing through the foil is

$$
\Delta T=\frac{4 N \mathcal{E}}{c \rho d A}=2913 \mathrm{~K}
$$

Thus if the foil starts out at $300 \mathrm{~K}$, its temperature will increase to $3213 \mathrm{~K}$ which is below but getting close to the melting point. The rate of temperature rise is

$$
\mathcal{K}=\frac{\Delta T}{\tau}=2158 \mathrm{~K} / \mathrm{ms}
$$




\section{Radiative Cooling during Energy Deposition}

We have the following numbers:

1. The emissivity of tungsten at $3600 \mathrm{~K}$ is [8]

$$
\epsilon=0.35
$$

2. The Stefan-Boltzmann constant is

$$
\sigma=5.67 \times 10^{-8} \mathrm{~W} \mathrm{~m}^{-2} \mathrm{~K}^{-4} .
$$

The rate at which energy is radiated from the foil is given by the Stefan-Boltzmann law [8]

$$
P=S \epsilon \sigma\left(T^{4}-T_{W}^{4}\right)
$$

where $T$ is the foil temperature and $T_{W}$ is the temperature of the vacuum chamber wall. Here

$$
S=2 A
$$

is the total surface area of the foil. (The foil has two sides of area $A$. We neglect the area of the foil edges.) As before, we take $A=2.0 \mathrm{~mm}^{2}$.

Thus, as energy is deposited during collisions with gold ions, the rate of temperature change of the portion of foil exposed to beam is

$$
\frac{d T}{d t}=\mathcal{K}-\frac{P}{c \rho d A}=\mathcal{K}-\frac{2 \epsilon \sigma}{c \rho d}\left(T^{4}-T_{W}^{4}\right)
$$

where $\mathcal{K}$ is given by (70). Defining

$$
\mathcal{C}=\frac{2 \epsilon \sigma}{c \rho d}, \quad \mathcal{A}^{4}=T_{W}^{4}+\frac{\mathcal{K}}{\mathcal{C}}
$$

we have

$$
\frac{d T}{d t}=-\mathcal{C}\left(T^{4}-\mathcal{A}^{4}\right)
$$

and integrating we obtain

$$
\int_{T_{1}}^{T_{2}} \frac{d T}{T^{4}-\mathcal{A}^{4}}=-\mathcal{C}\left(t_{2}-t_{1}\right) .
$$


Using

$$
\int \frac{d x}{a^{4}-x^{4}}=\frac{1}{4 a^{3}} \ln \left|\frac{a+x}{a-x}\right|+\frac{1}{2 a^{3}} \arctan \left(\frac{x}{a}\right)
$$

we then have

$$
\begin{aligned}
4 \mathcal{A}^{3} \mathcal{C}\left(t_{2}-t_{1}\right) & =\ln \left|\frac{\mathcal{A}+T_{2}}{\mathcal{A}-T_{2}}\right|+2 \arctan \left(\frac{T_{2}}{\mathcal{A}}\right) \\
& -\ln \left|\frac{\mathcal{A}+T_{1}}{\mathcal{A}-T_{1}}\right|-2 \arctan \left(\frac{T_{1}}{\mathcal{A}}\right) \\
\exp \left\{4 \mathcal{A}^{3} \mathcal{C}\left(t_{2}-t_{1}\right)\right\} & =\left|\frac{\mathcal{A}+T_{2}}{\mathcal{A}-T_{2}}\right|\left|\frac{\mathcal{A}-T_{1}}{\mathcal{A}+T_{1}}\right| \exp \left\{2\left(\theta_{2}-\theta_{1}\right)\right\}
\end{aligned}
$$

and

$$
\left|\frac{\mathcal{A}+T_{2}}{\mathcal{A}-T_{2}}\right| \exp \left\{2 \theta_{2}\right\}=\left|\frac{\mathcal{A}+T_{1}}{\mathcal{A}-T_{1}}\right| \exp \left\{2 \theta_{1}+4 \mathcal{A}^{3} \mathcal{C}\left(t_{2}-t_{1}\right)\right\}
$$

where

$$
\theta_{1}=\arctan \left(\frac{T_{1}}{\mathcal{A}}\right), \quad \theta_{2}=\arctan \left(\frac{T_{2}}{\mathcal{A}}\right) .
$$

Given $\mathcal{A}, T_{1}, \mathcal{C}$ and $t_{2}-t_{1}$ we can evaluate the right-hand side of (82) and then solve for $T_{2}$. We have

$$
\mathcal{C}=6.13404 \times 10^{-10}
$$

and taking

$$
T_{W}=300 \mathrm{~K}, \quad t_{2}-t_{1}=\tau
$$

we have

$$
\mathcal{A}=7701.35 \mathrm{~K}
$$

Then taking

$$
T_{1}=539.416 \mathrm{~K}
$$

we obtain

$$
\left|\frac{\mathcal{A}+T_{2}}{\mathcal{A}-T_{2}}\right| \exp \left\{2 \theta_{2}\right\}=6.00852 .
$$

Solving this for $T_{2}$ gives

$$
T_{2}=3425.08 \mathrm{~K}
$$

Here we have taken $T_{1}=539.416 \mathrm{~K}$ because, as shown in the next section, this is the temperature to which the exposed area of the foil will cool before the next energy deposition. (If an initial lower temperature is taken for $T_{1}$ then after a number of heating and radiative cooling cycles, an equilibrium is reached in which the foil cools to $539.416 \mathrm{~K}$ just before the next deposition of energy.) 


\section{Radiative Cooling after Energy Deposition}

After the short period ( $\tau=1.35 \mathrm{~ms}$ ) of energy deposition, the foil cools until the next deposition of energy. We shall take the AGS repetition period to be

$$
\mathcal{T}=3.6 \mathrm{~s} .
$$

The cooling time is nearly $\mathcal{T}$ and during this time we have $\mathcal{K}=0$. The second of Eqs. (76) then becomes

$$
\mathcal{A}=T_{W}
$$

and Eq. (82) becomes

$$
\left|\frac{T_{W}+T_{2}}{T_{W}-T_{2}}\right| \exp \left\{2 \theta_{2}\right\}=\left|\frac{T_{W}+T_{1}}{T_{W}-T_{1}}\right| \exp \left\{2 \theta_{1}+4 T_{W}^{3} \mathcal{C}\left(t_{2}-t_{1}\right)\right\}
$$

where

$$
\theta_{1}=\arctan \left(\frac{T_{1}}{T_{W}}\right), \quad \theta_{2}=\arctan \left(\frac{T_{2}}{T_{W}}\right)
$$

Taking

$$
T_{W}=300 \mathrm{~K}, \quad T_{1}=3425.08 \mathrm{~K}, \quad t_{2}-t_{1}=\mathcal{T}
$$

we then have

$$
\left|\frac{T_{W}+T_{2}}{T_{W}-T_{2}}\right| \exp \left\{2 \theta_{2}\right\}=29.3996
$$

and solving for $T_{2}$ we obtain

$$
T_{2}=539.416 \mathrm{~K}
$$

Thus, for each AGS cycle the foil temperature rises from $539.416 \mathrm{~K}$ to $3425.08 \mathrm{~K}$ during energy deposition and then cools back down to 539.416 before the next energy deposition. The peak temperature here is below but close to the melting point. This shows that the part of the foil that is exposed to beam can easily melt (assuming only radiative cooling) if the area $A$ is much smaller than the assumed $2.0 \mathrm{~mm}^{2}$. We must therefore consider cooling due to conduction of heat in the foil.

\section{Heat Flow in the Foil}

We have the following numbers: 
1. The thermal conductivity of tungsten at $300 \mathrm{~K}$ is $K=174 \mathrm{~W} /(\mathrm{mK})$.

2. The thermal conductivity at $1800 \mathrm{~K}$ is $K=100 \mathrm{~W} /(\mathrm{mK})$.

3. The foil thickness is $d=0.0254 \mathrm{~mm}$.

4. The beam width on the foil is $w=0.05 \mathrm{~mm}$.

5. The beam height on the foil is $h=10 \mathrm{~mm}$.

6. The foil area exposed to beam is $A=w h$.

7. The corresponding area of the foil edge is $a=d h$.

8. The time interval of energy deposition is $\tau=1.35 \mathrm{~ms}$.

9. The instantaneous power is $P=4 N \mathcal{E} / \tau=279$ watts.

The fundamental equation for heat flow is [9]

$$
\nabla \cdot \mathbf{h}+\frac{\partial u}{\partial t}=S
$$

where $u$ is the internal energy per unit volume, $\mathbf{h}$ is the heat energy flowing per unit time through a unit area perpendicular to the flow, and $S$ is the heat generated per unit volume per unit time. (This equation is simply a statement of the conservation of energy.) The heat flow vector $\mathbf{h}$ and the change in internal energy $u$ are related to the change in temperature by

$$
\mathbf{h}=-K \nabla T
$$

and

$$
\frac{\partial u}{\partial t}=c \rho \frac{\partial T}{\partial t}
$$

where $K$ is the thermal conductivity, $c$ is the specific heat, and $\rho$ is the material density. The temperature $T$ is a function of coordinates $x, y, z$ and time $t$. We shall assume that $K$ is constant throughout the foil. This gives

$$
\nabla \cdot \mathbf{h}=-K \nabla^{2} T
$$

and we have

$$
\nabla^{2} T-\frac{c \rho}{K} \frac{\partial T}{\partial t}=-\frac{S}{K}
$$


We assume further that the temperature depends only on $x$ and $t$. This gives the one-dimensional equations

$$
\frac{\partial^{2} T}{\partial x^{2}}-\frac{1}{\alpha} \frac{\partial T}{\partial t}=-\frac{S}{K}
$$

and

$$
h(x, t)=-K \frac{\partial T}{\partial x}
$$

where

$$
\alpha=\frac{K}{c \rho} .
$$

Here $x$ is the distance into the foil from the foil edge. The source term $S$ is nonzero only for $0 \leq x \leq w$ and $0 \leq t \leq \tau$, where $w$ is the hit width on the foil and $\tau$ is the energy deposition time. We shall assume that the foil has uniform temperature $T_{0}$ at time $t=0$. We assume further that there is no heat flow into the foil edge. Thus

$$
T(x, 0)=T_{0}, \quad h(0, t)=0 .
$$

For the numbers given above we have

$$
S=\frac{P}{d A}=\frac{4 N \mathcal{E}}{d A \tau}=22000 \mathrm{~W} / \mathrm{mm}^{3} .
$$

\section{Solution of the Heat Flow Equations}

The analytical solution of (102) that satisfies the desired boundary conditions is given by Carslaw and Jaeger [10]. For $0<x<w$ the temperature of the foil is

$$
T(x, t)=T_{0}+\frac{\alpha S t}{K}\left\{1-2 \mathrm{i}^{2} \operatorname{erfc}\left(\frac{w-x}{\sqrt{4 \alpha t}}\right)-2 \mathrm{i}^{2} \operatorname{erfc}\left(\frac{w+x}{\sqrt{4 \alpha t}}\right)\right\}
$$

and for $x>w$,

$$
T(x, t)=T_{0}+\frac{\alpha S t}{K}\left\{2 \mathrm{i}^{2} \operatorname{erfc}\left(\frac{x-w}{\sqrt{4 \alpha t}}\right)-2 \mathrm{i}^{2} \operatorname{erfc}\left(\frac{x+w}{\sqrt{4 \alpha t}}\right)\right\} .
$$

Here

$$
\mathrm{i}^{2} \operatorname{erfc} y=\frac{1}{4}\left\{\left(1+2 y^{2}\right) \operatorname{erfc} y-\frac{2}{\sqrt{\pi}} y \exp \left(-y^{2}\right)\right\}
$$


where

$$
\operatorname{erfc} y=1-\operatorname{erf} y
$$

and

$$
\operatorname{erf} y=\frac{2}{\sqrt{\pi}} \int_{0}^{y} \exp \left(-s^{2}\right) d s .
$$

Note that

$$
\mathrm{i}^{2} \operatorname{erfc} 0=\frac{1}{4}
$$

which shows that $T(x, t)$ is continuous at $x=w$.

The functions $\mathrm{i}^{n} \operatorname{erfc} y$ are defined by

$$
\mathrm{i}^{n} \operatorname{erfc} y=\int_{y}^{\infty} \mathrm{i}^{n-1} \operatorname{erfc} s d s, \quad n=1,2,3, \ldots
$$

with

$$
\mathrm{i}^{0} \operatorname{erfc} y=\operatorname{erfc} y \text {. }
$$

These satisfy the recurrence formula

$$
2 n \mathrm{i}^{n} \operatorname{erfc} y=\mathrm{i}^{n-2} \operatorname{erfc} y-2 y \mathrm{i}^{n-1} \operatorname{erfc} y
$$

and the differential equation

$$
\frac{d^{2} F}{d y^{2}}+2 y \frac{d F}{d y}-2 n F=0
$$

where $F=\mathrm{i}^{n} \operatorname{erfc} y$. For the case $n=2$ we have

$$
F(y)=\mathrm{i}^{2} \operatorname{erfc} y
$$

and

$$
F^{\prime \prime}+2 y F^{\prime}-4 F=0
$$

where

$$
F^{\prime \prime}=\frac{d^{2} F}{d y^{2}}, \quad F^{\prime}=\frac{d F}{d y} .
$$

To verify that (107) and (108) satisfy (102), we define

$$
G(x, t)=T_{0}+\frac{S \alpha t}{K}+\alpha t F(g(x, t))
$$

where

$$
g(x, t)=\frac{a+b x}{\sqrt{4 \alpha t}}, \quad b^{2}=1 .
$$


Then

$$
\begin{gathered}
\frac{\partial g}{\partial x}=\frac{b}{\sqrt{4 \alpha t}}, \quad \frac{\partial^{2} g}{\partial x^{2}}=0, \quad \frac{\partial g}{\partial t}=-\frac{g}{2 t} \\
\frac{\partial G}{\partial x}=\alpha t \frac{\partial g}{\partial x} F^{\prime}=\frac{b F^{\prime}}{\sqrt{4 \alpha t}} \alpha t \\
\frac{\partial^{2} G}{\partial x^{2}}=\alpha t\left(\frac{\partial g}{\partial x}\right)^{2} F^{\prime \prime}=\frac{b^{2} \alpha t}{4 \alpha t} F^{\prime \prime}=\frac{1}{4} F^{\prime \prime} \\
\frac{\partial G}{\partial t}=\frac{\alpha S}{K}+\alpha F+\alpha t \frac{\partial g}{\partial t} F^{\prime}=\alpha\left\{\frac{S}{K}+F-\frac{g}{2} F^{\prime}\right\}
\end{gathered}
$$

and we have

$$
\frac{\partial^{2} G}{\partial x^{2}}-\frac{1}{\alpha} \frac{\partial G}{\partial t}=\frac{1}{4}\left\{F^{\prime \prime}-4 F+2 g F^{\prime}\right\}-\frac{S}{K}
$$

Thus using (118) we have

$$
\frac{\partial^{2} G}{\partial x^{2}}-\frac{1}{\alpha} \frac{\partial G}{\partial t}=-\frac{S}{K}
$$

and it follows that (107) and (108) satisfy (102).

Now the heat flow in the foil is given by (103), and using (123) and

$$
F^{\prime}(y)=-\operatorname{ierfc}(y)
$$

we have, for $0 \leq x \leq w$,

$$
h(x, t)=S(\alpha t)^{3 / 2}\left\{\operatorname{ierfc}\left(\frac{w-x}{\sqrt{4 \alpha t}}\right)-\operatorname{ierfc}\left(\frac{w+x}{\sqrt{4 \alpha t}}\right)\right\}
$$

where

$$
\operatorname{ierfc}(y)=\frac{1}{\sqrt{\pi}} \exp \left(-y^{2}\right)-y \operatorname{erfc} y .
$$

Here we see that $h(0, t)=0$ as required.

For $w \leq x$ we have

$$
h(x, t)=S(\alpha t)^{3 / 2}\left\{\operatorname{ierfc}\left(\frac{x-w}{\sqrt{4 \alpha t}}\right)-\operatorname{ierfc}\left(\frac{x+w}{\sqrt{4 \alpha t}}\right)\right\} .
$$

This shows that $h(x, t)$ is continuous at $x=w$ as required. 
Figure 46 shows $T(x, \tau)$ obtained from (107) and (108) with $w=0.05$ $\mathrm{mm}, h=10 \mathrm{~mm}$ and initial temperature $T_{0}=300 \mathrm{~K}$. The energy deposition time $\tau$ is $1.35,2.7$, and $5.4 \mathrm{~ms}$ respectively for the upper, middle, and lower curves. Figures 47, 48, and 49 show curves with the same $h, T_{0}$ and energy deposition times, but with hit widths $w=0.01$, 0.005 , and $0.001 \mathrm{~mm}$ respectively. Note that even as the width $w$ becomes very small, the temperature at the foil edge stays below the melting point of $3687 \mathrm{~K}$.

\section{Temperature at the Foil Edge}

The analytical expression for the temperature at the foil edge is obtained by setting $x=0$ in (107). This gives

$$
T(0, t)=T_{0}+\frac{\alpha S t}{K}\left\{1-4 \mathrm{i}^{2} \operatorname{erfc}\left(\frac{w}{\sqrt{4 \alpha t}}\right)\right\}
$$

where

$$
4 \mathrm{i}^{2} \operatorname{erfc} y=\left(1+2 y^{2}\right) \operatorname{erfc} y-\frac{2}{\sqrt{\pi}} y \exp \left(-y^{2}\right)
$$

and

$$
\operatorname{erfc} y=1-\frac{2}{\sqrt{\pi}} \int_{0}^{y} \exp \left(-s^{2}\right) d s \text {. }
$$

Table 2 lists the temperatures $T(0, \tau)$ at the foil edge for the curves in Figures 46, 47, 48, and 49. Temperatures $T_{1}, T_{2}, T_{3}$ and $T_{4}$ are those obtained with $w=0.05,0.01,0.005$, and $0.001 \mathrm{~mm}$ respectively.

Table 2: Temperature at the Foil Edge

\begin{tabular}{|c|c|c|c|c|c|}
\hline$\tau$ & $\sqrt{4 \alpha \tau}$ & $T_{1}(0, \tau)$ & $T_{2}(0, \tau)$ & $T_{3}(0, \tau)$ & $T_{4}(0, \tau)$ \\
\hline $\mathrm{ms}$ & $\mathrm{mm}$ & ${ }^{\circ} \mathrm{K}$ & ${ }^{\circ} \mathrm{K}$ & ${ }^{\circ} \mathrm{K}$ & ${ }^{\circ} \mathrm{K}$ \\
\hline 1.35 & 0.460 & 2892 & 3101 & 3128 & 3150 \\
2.70 & 0.651 & 2186 & 2292 & 2306 & 2317 \\
5.40 & 0.921 & 1661 & 1714 & 1721 & 1727 \\
\hline
\end{tabular}

There are two important features here. The first is that for each value of $w$ the temperature is significantly reduced by increasing the time $\tau$ over which the fixed energy $4 N \mathcal{E}$ is deposited. The second is that for each value 
of $\tau$ the temperature increases toward an upper limit as $w$ gets smaller. This will be shown rigorously in the next section.

\section{The Limit of very Small Hit Width}

For $|y| \ll 1$ we have

$$
1-4 \mathrm{i}^{2} \operatorname{erfc} y=\frac{4}{\sqrt{\pi}} y-2 y^{2}
$$

and therefore for

$$
w \ll \sqrt{4 \alpha t}
$$

the temperature at the foil edge becomes

$$
T(0, t)=T_{0}+\frac{S w}{K}\left\{\sqrt{\frac{4 \alpha t}{\pi}}-\frac{w}{2}\right\} .
$$

Here

$$
\frac{S w}{K}=\frac{P w}{d A K}=\frac{P}{a K}=\frac{4 N \mathcal{E}}{a \tau K}
$$

which is independent of $w$. For $t=\tau$ we then have

$$
T(0, \tau)=T_{0}+\frac{4 N \mathcal{E}}{a K}\left\{\sqrt{\frac{4 \alpha}{\pi \tau}}-\frac{w}{2 \tau}\right\}
$$

which we can write as

$$
\begin{gathered}
T(0, \tau)=T_{0}+\frac{4 N \mathcal{E}}{a K} \frac{4 \alpha}{\sqrt{\pi}}\left\{\sqrt{\frac{1}{4 \alpha \tau}}-\frac{\sqrt{\pi}}{2} \frac{w}{4 \alpha \tau}\right\} \\
T(0, \tau)=T_{0}+\frac{4 N \mathcal{E}}{a K} \frac{4 \alpha}{\sqrt{\pi}} \sqrt{\frac{1}{4 \alpha \tau}}\left\{1-\frac{\sqrt{\pi}}{2} \frac{w}{\sqrt{4 \alpha \tau}}\right\} .
\end{gathered}
$$

In the limit of vanishingly small $w$ this becomes simply

$$
T(0, \tau)=T_{0}+\frac{4 N \mathcal{E}}{a K} \frac{4 \alpha}{\sqrt{\pi}} \sqrt{\frac{1}{4 \alpha \tau}}
$$

which shows that the temperature increase at the foil edge goes like $1 / \sqrt{\tau}$. Putting in numbers

1. $4 N \mathcal{E}=0.377$ Joules 
2. $a=d h=0.254 \mathrm{~mm}^{2}$

3. $K=100 \mathrm{~W} /(\mathrm{mK})$

4. $\tau=1.35 \mathrm{~ms}$

5. $T_{0}=300 \mathrm{~K}$

we obtain

$$
\begin{gathered}
\frac{4 N \mathcal{E}}{a K} \frac{4 \alpha}{\sqrt{\pi}}=1314.8 \mathrm{~mm} \mathrm{~K} \\
\sqrt{4 \alpha \tau}=0.4604 \mathrm{~mm} \\
\frac{4 N \mathcal{E}}{a K} \frac{4 \alpha}{\sqrt{\pi}} \sqrt{\frac{1}{4 \alpha \tau}}=2855.8 \mathrm{~K}
\end{gathered}
$$

and

$$
T(0, \tau)=T_{0}+\frac{4 N \mathcal{E}}{a K} \frac{4 \alpha}{\sqrt{\pi}} \sqrt{\frac{1}{4 \alpha \tau}}=3155.8 \mathrm{~K} .
$$

Thus, for energy deposition times $\tau$ greater than or equal to $1.35 \mathrm{~ms}$, the foil edge stays below the melting point of $3687 \mathrm{~K}$ even in the extreme case of the hit width going to zero.

\section{The Case of Heat Flowing into the Foil Edge}

Instead of considering the problem in which heat is generated between $x=0$ and $x=w$, we consider the problem in which an equivalent amount of heat flows into the foil through the edge at $x=0$. Following the treatment of Eckert and Drake [11] we have

$$
\begin{gathered}
h(x, t)=-K \frac{\partial T}{\partial x} \\
\frac{\partial h}{\partial t}=-K \frac{\partial^{2} T}{\partial t \partial x} \\
\frac{\partial^{2} T}{\partial x^{2}}-\frac{c \rho}{K} \frac{\partial T}{\partial t}=0
\end{gathered}
$$




$$
\begin{aligned}
& \frac{\partial h}{\partial x}=-K \frac{\partial^{2} T}{\partial x^{2}}=-c \rho \frac{\partial T}{\partial t} \\
& \frac{\partial^{2} h}{\partial x^{2}}=-c \rho \frac{\partial^{2} T}{\partial x \partial t}=\frac{c \rho}{K} \frac{\partial h}{\partial t}
\end{aligned}
$$

and

$$
\frac{\partial h}{\partial t}-\alpha \frac{\partial^{2} h}{\partial x^{2}}=0
$$

where

$$
\alpha=\frac{K}{c \rho} .
$$

We wish to find a solution of (152) subject to the boundary conditions

$$
\begin{gathered}
h(x, t)=0, \quad x>0, \quad t=0 \\
h(x, t)=h_{0}, \quad x=0, \quad 0<t \leq \tau
\end{gathered}
$$

where

$$
h_{0}=\frac{P}{a}=\frac{4 N \mathcal{E}}{a \tau} .
$$

The solution is

$$
h(x, t)=h_{0}\left\{1-\operatorname{erf} \frac{x}{\sqrt{4 \alpha t}}\right\}
$$

where

$$
\operatorname{erf} y=\frac{2}{\sqrt{\pi}} \int_{0}^{y} \exp \left(-s^{2}\right) d s .
$$

This can be verified using

$$
\frac{d}{d y} \operatorname{erf} y=\frac{2}{\sqrt{\pi}} \exp \left(-y^{2}\right) .
$$

We have

$$
\begin{gathered}
\frac{\partial h}{\partial x}=-\frac{2 h_{0}}{\sqrt{4 \pi \alpha t}} \exp \left(-\frac{x^{2}}{4 \alpha t}\right) \\
\frac{\partial^{2} h}{\partial x^{2}}=\frac{2 h_{0}}{\sqrt{4 \pi \alpha t}} \frac{2 x}{4 \alpha t} \exp \left(-\frac{x^{2}}{4 \alpha t}\right) \\
\alpha \frac{\partial^{2} h}{\partial x^{2}}=\frac{h_{0} x}{t \sqrt{4 \pi \alpha t}} \exp \left(-\frac{x^{2}}{4 \alpha t}\right)
\end{gathered}
$$




$$
\frac{\partial h}{\partial t}=-\frac{h_{0} x}{\sqrt{4 \alpha}}\left\{-\frac{1}{2} t^{-3 / 2}\right\} \frac{2}{\sqrt{\pi}} \exp \left(-\frac{x^{2}}{4 \alpha t}\right)
$$

and

$$
\frac{\partial h}{\partial t}=\frac{h_{0} x}{t \sqrt{4 \pi \alpha t}} \exp \left(-\frac{x^{2}}{4 \alpha t}\right)=\alpha \frac{\partial^{2} h}{\partial x^{2}}
$$

as desired. Furthermore, using

$$
\operatorname{erf}(0)=0, \quad \operatorname{erf}(\infty)=1
$$

we have

$$
h(0, t)=h_{0}, \quad h(x, 0)=0
$$

which are the desired boundary conditions.

Now, integrating (147) we have

$$
\begin{gathered}
-K\left\{T_{0}-T(x, t)\right\}=\int_{x}^{\infty} h(s, t) d s \\
T(x, t)=T_{0}+\frac{h_{0}}{K} \int_{x}^{\infty} \operatorname{erfc}\left(\frac{s}{\sqrt{4 \alpha t}}\right) d s
\end{gathered}
$$

where

$$
T_{0}=T(\infty, t)
$$

and

$$
\operatorname{erfc} y=1-\operatorname{erf} y \text {. }
$$

Defining

$$
u=\frac{s}{\sqrt{4 \alpha t}}
$$

we have

$$
d s=d u \sqrt{4 \alpha t}
$$

and

$$
T(x, t)=T_{0}+\frac{h_{0}}{K} \sqrt{4 \alpha t} \int_{y}^{\infty} \operatorname{erfc} u d u
$$

where

$$
y=\frac{x}{\sqrt{4 \alpha t}} .
$$

Using

$$
\int_{y}^{\infty} \operatorname{erfc} u d u=\frac{1}{\sqrt{\pi}} \exp \left(-y^{2}\right)-y \operatorname{erfc} y
$$


we then have

$$
T(x, t)=T_{0}+\frac{h_{0}}{K} \sqrt{\frac{4 \alpha t}{\pi}} F(x, t)
$$

where

$$
F(x, t)=\exp \left(-\frac{x^{2}}{4 \alpha t}\right)-\sqrt{\frac{\pi x^{2}}{4 \alpha t}} \operatorname{erfc}\left(\frac{x}{\sqrt{4 \alpha t}}\right) .
$$

Here

$$
\frac{h_{0}}{K}=\frac{P}{a K}=\frac{4 N \mathcal{E}}{a \tau K}
$$

and we have

$$
\frac{h_{0}}{K} \sqrt{\frac{4 \alpha \tau}{\pi}}=\frac{4 N \mathcal{E}}{a K} \frac{4 \alpha}{\sqrt{\pi}} \sqrt{\frac{1}{4 \alpha \tau}} .
$$

We also have

$$
F(0, t)=1
$$

The temperature at the foil edge $(x=0)$ at time $t=\tau$ is then

$$
T(0, \tau)=T_{0}+\frac{4 N \mathcal{E}}{a K} \frac{4 \alpha}{\sqrt{\pi}} \sqrt{\frac{1}{4 \alpha \tau}}
$$

in agreement with (142).

\section{Inclined Plunging Stripper}

In the above work we assumed that the stripper edge makes an angle $\theta=90^{\circ}$ with respect to the direction of the plunging motion. We took the vertical extent of the beam on the stripper to be $h=10 \mathrm{~mm}$ as illustrated in Figure 33. However, if the angle of the edge is not exactly $90^{\circ}$ then, because of the vertical oscillations of the beam particles, the particles will hit just half of the length of the projection of the beam onto the edge. The extent of particle hits along the edge is then $h /(2 \sin \theta)$. This is illustrated in Figures $\mathbf{5 0}$ and $\mathbf{5 1}$ where $\theta=60^{\circ}$ and $30^{\circ}$ respectively. For the case in which $\theta=30^{\circ}$ we have $h /(2 \sin \theta)=h$ and we recover $h$ as the extent of hits along the edge.

To verify that these ideas are correct, a simulation was done in which the initial distribution of beam particles was the largest ball (in normalized $X$, $X^{\prime}, Y, Y^{\prime}$ coordinates) contained in a four-dimensional cube uniformly

populated with $40^{4}$ particles. Beam half widths were taken to be $\sqrt{\epsilon \beta}=2.7$ and $5.0 \mathrm{~mm}$ in the horizontal and vertical planes respectively. The stripper 
was moved into the circulating beam in horizontal steps of $0.002 \sqrt{\epsilon \beta}$ per turn $(2.7 \mu \mathrm{s})$ which gives a speed of $2.0 \mathrm{~mm} / \mathrm{ms}$. The times over which hits occur on the stripper are then $1.35,1.98$, and $4.54 \mathrm{~ms}$ for $\theta=90,60$, and 30 degrees respectively. During these times the stripper moves 2.70, 3.95 and $9.07 \mathrm{~mm}$ respectively in the horizontal direction. The horizontal and vertical tunes were taken to be $Q_{H}=0.281$ and $Q_{V}=0.221$. As shown in the previous sections, the longer energy deposition times obtained for $\theta=60$ and 30 degrees give a lower temperature at the foil edge.

Figures $\mathbf{5 2}$ and $\mathbf{5 3}$ show the distribution of hits on the stripper. The Red, Blue and Green histograms were obtained with $\theta=90,60$, and 30 degrees respectively. Note that for the red histogram in Figure 52, $\theta$ was exactly 90 degrees, so the extent of the particle hits along the edge is $h=10 \mathrm{~mm}$ (not $h / 2=5 \mathrm{~mm}$ ). For the green histogram, the extent of hits along the edge is $h /(2 \sin \theta)=h$ as expected for $\theta=30$ degrees. In Figure 53 we see that the hits are spread over a significantly greater distance from the foil edge for $\theta=60$ and 30 degrees. This again gives a lower temperature at the foil edge.

The use of an inclined stripper was proposed and advocated by Peter Thieberger. His design with $\theta=30$ degrees is shown in Figures 54 and 55. This is the design that was built by George Mahler and installed in the J7 straight. There are two plunging strippers here; one is plunged from the inside side of the ring and the other from the outside. This allows for a working spare in the event that one of the strippers or plunging mechanisms fails. Each stripper is driven by a programmable linear motor as described in [12].

An added advantage of the inclined stripper is that the foil edge can be viewed from above with a camera. With $6.0 \times 10^{9}$ gold ions circulating in the machine at extraction energy, a bright flash is seen as the stripper is plunged into the beam. Figures $\mathbf{5 6}$ and $\mathbf{5 7}$ show the inside plunging stripper after several weeks of running. Here one clearly sees a gouged out area where the beam has hit the foil. As of this writing it is not known whether this is due to melting or evaporation of the tungsten or to some other process.

\section{Stay-Clear Region in the J7 Straight}

The horizontal limiting aperture in AGS occurs at the A5 injection kicker which has a half-aperture of 2.532 inches [5]. Taking $\beta_{x}=23 \mathrm{~m}$ at the 
kicker gives horizontal acceptance $\pi e_{x}=180 \pi \mathrm{mm}$ mrad.

The vertical limiting aperture is in the number 11 magnet of each superperiod. Here the vertical half-aperture is 1.531 inches [4]. Taking $\beta_{y}=23$ here gives vertical acceptance $\pi e_{y}=66 \pi \mathrm{mm} \mathrm{mrad}$.

Thus when the J7 stripper is not being plunged into the aperture, it must stay clear of the region defined by

$$
-\sqrt{e_{x} \beta_{H}} \leq X \leq \sqrt{e_{x} \beta_{H}}
$$

and

$$
-\sqrt{e_{y} \beta_{V}} \leq Y \leq \sqrt{e_{y} \beta_{V}}
$$

where $\beta_{H}$ and $\beta_{V}$ are the horizontal and vertical beta functions at the stripper. Taking

$$
\beta_{H}=11 \mathrm{~m}, \quad \beta_{V}=21 \mathrm{~m}
$$

and using the values of $e_{x}$ and $e_{y}$ given above we then have

$$
|X| \leq 44 \mathrm{~mm}, \quad|Y| \leq 37 \mathrm{~mm} .
$$

\section{Observed Loss Patterns with and without the Plunging Stripper.}

Figures $\mathbf{5 8}$ and $\mathbf{5 9}$ show the loss pattern in the AGS with some $3.5 \times 10^{9}$ gold ions being put into the J10 dump at extraction energy. (These are images taken from the 11 Dec 2009 Booster-Ags-Au-2010 elog.)

In Figure 58 the J7 stripper is not being plunged into the circulating beam. Here the loss has a peak value of about 1100 at the J12 loss monitor and a shoulder value of about 200 at J16 and J18. There is also a small loss registering at the L10 loss monitor. The Au79+ trajectory shown in Figures 9, 10, and $\mathbf{1 1}$ suggests a possible explanation for losses in the L9 straight.

In Figure 59 the J7 stripper is being plunged into the beam. Here the peak loss at the J12 monitor has been reduced to about 600 and the shoulder at J16 and J18 has come down to 50 or so. The loss at the L10 monitor has completely disappeared. 


\section{Energy Deposition in the J10 Dump}

We have the following numbers:

1. The kinetic energy of a single fully stripped gold ion at AGS extraction is

$$
W=197 \times 8.86=1745.42 \mathrm{GeV} .
$$

2. The kinetic energy of a proton with the same velocity as the gold ion is

$$
W_{p}=8.93 \mathrm{GeV} .
$$

3. The rate of energy loss of a proton passing through copper with kinetic energy $W_{p}$ is [13]

$$
-\frac{d E_{p}}{d x}=1.534 \mathrm{MeV} \mathrm{cm}^{2} / \mathrm{g}
$$

4. The rate of energy loss of a fully stripped gold ion $(Z=79)$ passing through copper with kinetic energy $8.86 \mathrm{GeV}$ per nucleon can then be obtained by scaling the Bethe-Bloch result for protons [14]. This gives

$$
-\frac{d E}{d x}=-Z^{2} \frac{d E_{p}}{d x}=9574 \mathrm{MeV} \mathrm{cm}^{2} / \mathrm{g} .
$$

5. This agrees fairly well with

$$
-\frac{d E}{d x}=9624 \mathrm{MeV} \mathrm{cm}^{2} / \mathrm{g}
$$

obtained from the computer code of J.P. Biersack and J.F.

Ziegler [7]. The projected range obtained by the code is $207.29 \mathrm{~mm}$.

6. The density of copper is $[6]$

$$
\rho=8.96 \mathrm{~g} / \mathrm{cm}^{3} .
$$

7. The specific heat of copper is $[6]$

$$
c=0.385 \mathrm{~J} /(\mathrm{gK}) .
$$

8. We assume that

$$
N=6.0 \times 10^{9}
$$

gold ions are put into the dump at extraction energy each AGS cycle. 
9. The total energy deposited into the dump per AGS cycle is then

$$
\mathcal{E}=N W=1678 \text { Joules. }
$$

10. We take the AGS cycle time to be

$$
\mathcal{T}=3.6 \mathrm{~s}
$$

11. This gives average power

$$
P=\mathcal{E} / \mathcal{T}=466 \text { watts. }
$$

The energy lost as $N$ fully stripped gold ions travel a small distance $d$ in copper is

$$
E=-N \frac{d E}{d x} \rho d
$$

If the ions are incident on surface area $A$, the energy is deposited in mass

$$
M=\rho A d
$$

and the resulting temperature increase (assuming no cooling) is

$$
\Delta T=\frac{E}{c M}=-\frac{N}{c A} \frac{d E}{d x} .
$$

In Section 10 we found that the trajectories of Au79+ ions that hit the upstream face of the dump are spread over a region that is some $4 \mathrm{~mm}$ wide. The beam height here is about $7 \mathrm{~mm}$. This gives $A=0.28 \mathrm{~cm}^{2}$ and

$$
\Delta T=86^{\circ} \mathrm{K} \text {. }
$$

Note that for $N_{p}$ protons moving at the same velocity as the gold ions we would have temperature increase

$$
\Delta T_{p}=-\frac{N_{p}}{c A} \frac{d E_{p}}{d x}=\frac{N_{p}}{N Z^{2}} \Delta T
$$

During the 1998 SEB Run [15] the peak intensity in AGS was

$$
N_{p}=7.1 \times 10^{13}
$$

protons per AGS cycle, which gives

$$
\frac{N_{p}}{N Z^{2}}=1.9
$$

This shows that the heating due to peak-intensity protons incident on the dump is only a factor of two higher than that due to peak-intensity gold ions. 


\section{Appendix}

Let

$$
\mathbf{X}_{0}=\left(\begin{array}{c}
X_{0} \\
X_{0}^{\prime}
\end{array}\right)
$$

where $X_{0}$ and $X_{0}^{\prime}$ are the horizontal position and angle of a beam particle at position $s_{0}$ along the reference orbit. Similarly let

$$
\mathbf{X}=\left(\begin{array}{c}
X \\
X^{\prime}
\end{array}\right)
$$

where $X$ and $X^{\prime}$ are the position and angle at $s$. Let $\Delta p / p$ be the momentum deviation parameter of the particle. We assume that a local orbit bump is produced by a series of point dipole kicks $\phi_{i}$ at positions $s_{i}$ along the reference orbit. Let

$$
\mathbf{T}_{i}=\left(\begin{array}{ccc}
a_{i} & b_{i} & e_{i} \\
f_{i} & g_{i} & e_{i}^{\prime} \\
0 & 0 & 1
\end{array}\right)=\left(\begin{array}{cc}
\mathbf{M}_{i} & \mathbf{e}_{i} \\
0 & 1
\end{array}\right)
$$

be the transfer matrix from $s_{i-1}$ to $s_{i}$. Here $\mathbf{M}_{i}$ and $\mathbf{e}_{i}$ are the submatrices

$$
\mathbf{M}_{i}=\left(\begin{array}{cc}
a_{i} & b_{i} \\
f_{i} & g_{i}
\end{array}\right), \quad \mathbf{e}_{i}=\left(\begin{array}{c}
e_{i} \\
e_{i}^{\prime}
\end{array}\right) .
$$

Let

$$
\begin{gathered}
\mathbf{Z}=\left(\begin{array}{c}
X \\
X^{\prime} \\
\Delta p / p
\end{array}\right)=\left(\begin{array}{c}
\mathbf{X} \\
\Delta p / p
\end{array}\right) \\
\mathbf{Z}_{0}=\left(\begin{array}{c}
X_{0} \\
X_{0}^{\prime} \\
\Delta p / p
\end{array}\right)=\left(\begin{array}{c}
\mathbf{X}_{0} \\
\Delta p / p
\end{array}\right)
\end{gathered}
$$

and

$$
\Theta_{i}=K\left(\begin{array}{c}
0 \\
\phi_{i} \\
0
\end{array}\right)=K\left(\begin{array}{c}
\Phi_{i} \\
0
\end{array}\right)
$$

where

$$
\Phi_{i}=\left(\begin{array}{c}
0 \\
\phi_{i}
\end{array}\right)
$$


and

$$
K=1-\frac{\Delta p}{p} .
$$

We then have

$$
\begin{aligned}
& \mathbf{Z}=\mathbf{T}_{N} \mathbf{T}_{N-1} \cdots \mathbf{T}_{1} \mathbf{Z}_{0} \\
& +\mathbf{T}_{N} \mathbf{T}_{N-1} \cdots \mathbf{T}_{2} \Theta_{1} \\
& +\mathbf{T}_{N} \mathbf{T}_{N-1} \cdots \mathbf{T}_{3} \Theta_{2} \\
& +\mathbf{T}_{N} \mathbf{T}_{N-1} \cdots \mathbf{T}_{4} \Theta_{3} \\
& \text { : } \\
& +\mathbf{T}_{N} \Theta_{N-1} \\
& \mathbf{T}_{2} \mathbf{T}_{1}=\left(\begin{array}{cc}
\mathbf{M}_{2} & \mathbf{e}_{2} \\
0 & 1
\end{array}\right)\left(\begin{array}{cc}
\mathbf{M}_{1} & \mathbf{e}_{1} \\
0 & 1
\end{array}\right) \\
& \mathbf{T}_{2} \mathbf{T}_{1}=\left(\begin{array}{cc}
\mathbf{M}_{2} \mathbf{M}_{1} & \mathbf{M}_{2} \mathbf{e}_{1}+\mathbf{e}_{2} \\
0 & 1
\end{array}\right) \\
& \mathbf{T}_{3} \mathbf{T}_{2} \mathbf{T}_{1}=\left(\begin{array}{cc}
\mathbf{M}_{3} & \mathbf{e}_{3} \\
0 & 1
\end{array}\right)\left(\begin{array}{cc}
\mathbf{M}_{2} \mathbf{M}_{1} & \mathbf{M}_{2} \mathbf{e}_{1}+\mathbf{e}_{2} \\
0 & 1
\end{array}\right) \\
& \mathbf{T}_{3} \mathbf{T}_{2} \mathbf{T}_{1}=\left(\begin{array}{cc}
\mathbf{M}_{3} \mathbf{M}_{2} \mathbf{M}_{1} & \mathbf{M}_{3} \mathbf{M}_{2} \mathbf{e}_{1}+\mathbf{M}_{3} \mathbf{e}_{2}+\mathbf{e}_{3} \\
0 & 1
\end{array}\right)
\end{aligned}
$$

and it follows that

$$
\begin{aligned}
\mathbf{X} & =\mathbf{M}_{N} \mathbf{M}_{N-1} \cdots \mathbf{M}_{1} \mathbf{X}_{0} \\
& +\mathbf{M}_{N} \mathbf{M}_{N-1} \cdots \mathbf{M}_{2}\left(\frac{\Delta p}{p} \mathbf{e}_{1}+K \Phi_{1}\right) \\
& +\mathbf{M}_{N} \mathbf{M}_{N-1} \cdots \mathbf{M}_{3}\left(\frac{\Delta p}{p} \mathbf{e}_{2}+K \Phi_{2}\right) \\
& +\mathbf{M}_{N} \mathbf{M}_{N-1} \cdots \mathbf{M}_{4}\left(\frac{\Delta p}{p} \mathbf{e}_{3}+K \Phi_{3}\right) \\
& \vdots \\
& +\mathbf{M}_{N}\left(\frac{\Delta p}{p} \mathbf{e}_{N-1}+K \Phi_{N-1}\right)+\frac{\Delta p}{p} \mathbf{e}_{N} .
\end{aligned}
$$


Let

$$
\mathbf{d}_{0}=\left(\begin{array}{c}
d_{0} \\
d_{0}^{\prime}
\end{array}\right), \quad \mathbf{d}=\left(\begin{array}{c}
d \\
d^{\prime}
\end{array}\right)
$$

where $d_{0}, d_{0}^{\prime}$ and $d, d^{\prime}$ are the closed orbit positions and angles at $s_{0}$ and $s$ respectively. Then

$$
\begin{aligned}
\mathbf{d} & =\mathbf{M}_{N} \mathbf{M}_{N-1} \cdots \mathbf{M}_{1} \mathbf{d}_{0} \\
& +\mathbf{M}_{N} \mathbf{M}_{N-1} \cdots \mathbf{M}_{2} \Phi_{1} \\
& +\mathbf{M}_{N} \mathbf{M}_{N-1} \cdots \mathbf{M}_{3} \Phi_{2} \\
& +\mathbf{M}_{N} \mathbf{M}_{N-1} \cdots \mathbf{M}_{4} \Phi_{3} \\
& \vdots \\
& +\mathbf{M}_{N} \Phi_{N-1} .
\end{aligned}
$$

Similarly, let

$$
\mathbf{D}_{0}=\left(\begin{array}{c}
D_{0} \\
D_{0}^{\prime}
\end{array}\right), \quad \mathbf{D}=\left(\begin{array}{c}
D \\
D^{\prime}
\end{array}\right)
$$

where $D_{0}, D_{0}^{\prime}$ and $D, D^{\prime}$ are the periodic dispersion functions at $s_{0}$ and $s$ respectively. Then

$$
\begin{aligned}
\mathbf{D} & =\mathbf{M}_{N} \mathbf{M}_{N-1} \cdots \mathbf{M}_{1} \mathbf{D}_{0} \\
& +\mathbf{M}_{N} \mathbf{M}_{N-1} \cdots \mathbf{M}_{2} \mathbf{e}_{1} \\
& +\mathbf{M}_{N} \mathbf{M}_{N-1} \cdots \mathbf{M}_{3} \mathbf{e}_{2} \\
& +\mathbf{M}_{N} \mathbf{M}_{N-1} \cdots \mathbf{M}_{4} \mathbf{e}_{3} \\
& \vdots \\
& +\mathbf{M}_{N} \mathbf{e}_{N-1}+\mathbf{e}_{N}
\end{aligned}
$$

Thus

$$
\mathbf{X}=\mathbf{M} \mathbf{X}_{0}+\frac{\Delta p}{p}\left(\mathbf{D}-\mathbf{M D}_{0}\right)+K\left(\mathbf{d}-\mathbf{M d}_{0}\right)
$$

where

$$
\mathbf{M}=\mathbf{M}_{N} \mathbf{M}_{N-1} \cdots \mathbf{M}_{1} .
$$

Rearranging terms we also have

$$
\mathbf{X}-\frac{\Delta p}{p} \mathbf{D}-K \mathbf{d}=\mathbf{M}\left\{\mathbf{X}_{0}-\frac{\Delta p}{p} \mathbf{D}_{0}-K \mathbf{d}_{0}\right\}
$$

which shows that the particle simply oscillates about the perturbed orbit $(\Delta p / p) \mathbf{D}+K \mathbf{d}$. 


\section{References}

[1] L.A. Ahrens, "AGS Au77+ Beam Dumping (making holes in the vacuum chambers)", RHIC Retreat 2008.

[2] L.A. Ahrens, Booster-AGS-Au-2010 elog (electronic log).

[3] C.J. Gardner, "Notes on Orbit Equations in the AGS", C-A/AP/Note 164, September 2004.

[4] P. Carolan, et al, "Another Look at Apertures in the AGS", AGS/AD/Tech Note 355, November 15, 1991.

[5] L.A. Ahrens and C.J. Gardner, "Determination of the AGS Injection Kicker Strength from Beam Measurements", C-A/AP/Note 91, December 2002.

[6] Periodic Table Online, CRC Press.

[7] J.P. Biersack and J.F. Ziegler, Computer code, Copyright 1984, 1989, 1992, 1998, 2008.

[8] M.W. Zemansky, Heat and Thermodynamics, 5th Edition, McGraw-Hill, pp. 99-103 (1968)

[9] R.P. Feynman, R.B. Leighton and M. Sands, The Feynman Lectures on Physics, Volume II, Addison-Wesley Publishing Company, 1964

[10] H.S. Carslaw and J.C. Jaeger, Conduction of Heat in Solids, Second Edition, Oxford University Press, p. 80 (2008)

[11] E.R.G. Eckert and R.M. Drake, Jr., Analysis of Heat and Mass Transfer, McGraw-Hill, Inc., pp. 168-169 (1972).

[12] E.R. Beadle, E.S. Rodger, R.E. Thern, "A Beam Scraper using a Linear Motor", PAC89, pp. 1474-1476

[13] M.J. Berger, J.S. Coursey, M.A. Zucker and J. Chang, "Stopping-Power and Range Tables for Electrons, Protons, and Helium Ions", www.nist.gov/physlab/data/star/index.cfm

[14] W.R. Leo, "Techniques for Nuclear and Particle Physics Experiments", Second Revised Edition, Springer-Verlag, 1994, pp. $24-28$. 
[15] L.A. Ahrens, J.M. Brennan, et al., "High Intensity Performance of the Brookhaven AGS", PAC99, pp. 614-616.

\section{$28 \quad$ Figures}




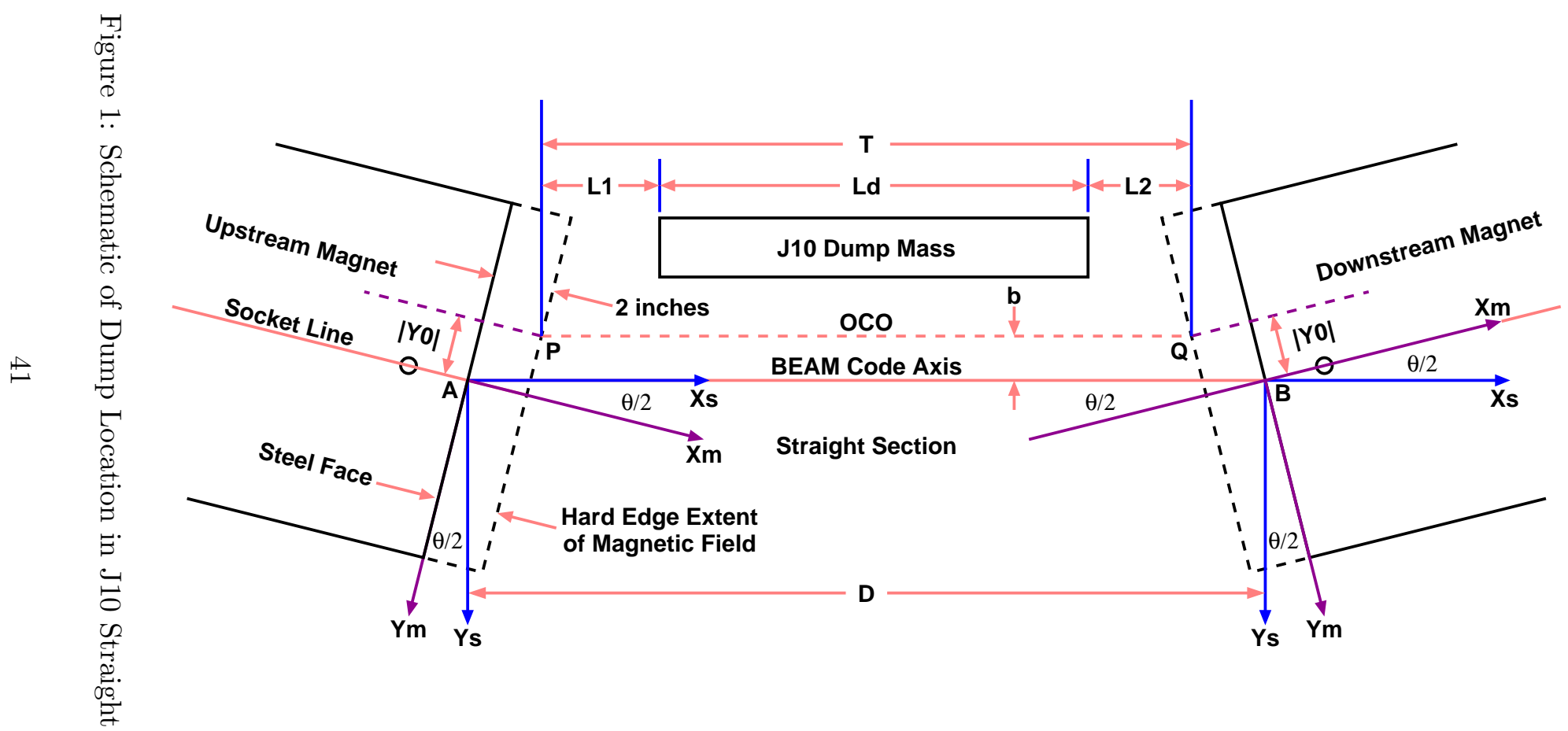




\section{AGS J10 Dump Bump}

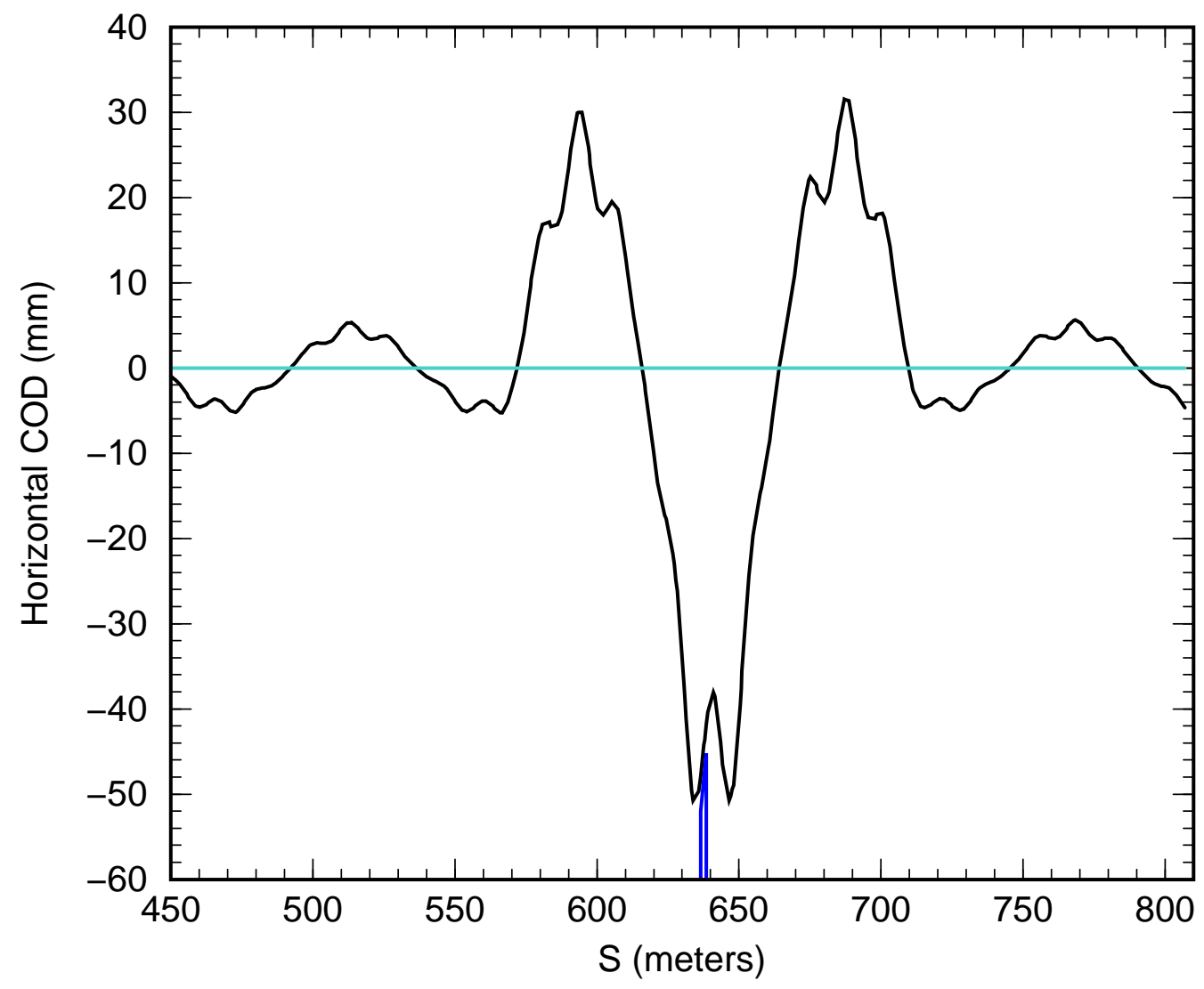

Figure 2: The black curve is the J10 dump bump. This is a standard AGS three-halves lambda bump obtained by exciting the backleg windings on four pairs of main dipole magnets. (In this case the I10, I11, J4, J5, J18, $\mathrm{J} 19$, K12, and K13 magnets.) Outside the bump region there is a residual orbit distortion that depends on the horizontal tune. The Blue lines show the outline of the J10 dump. 


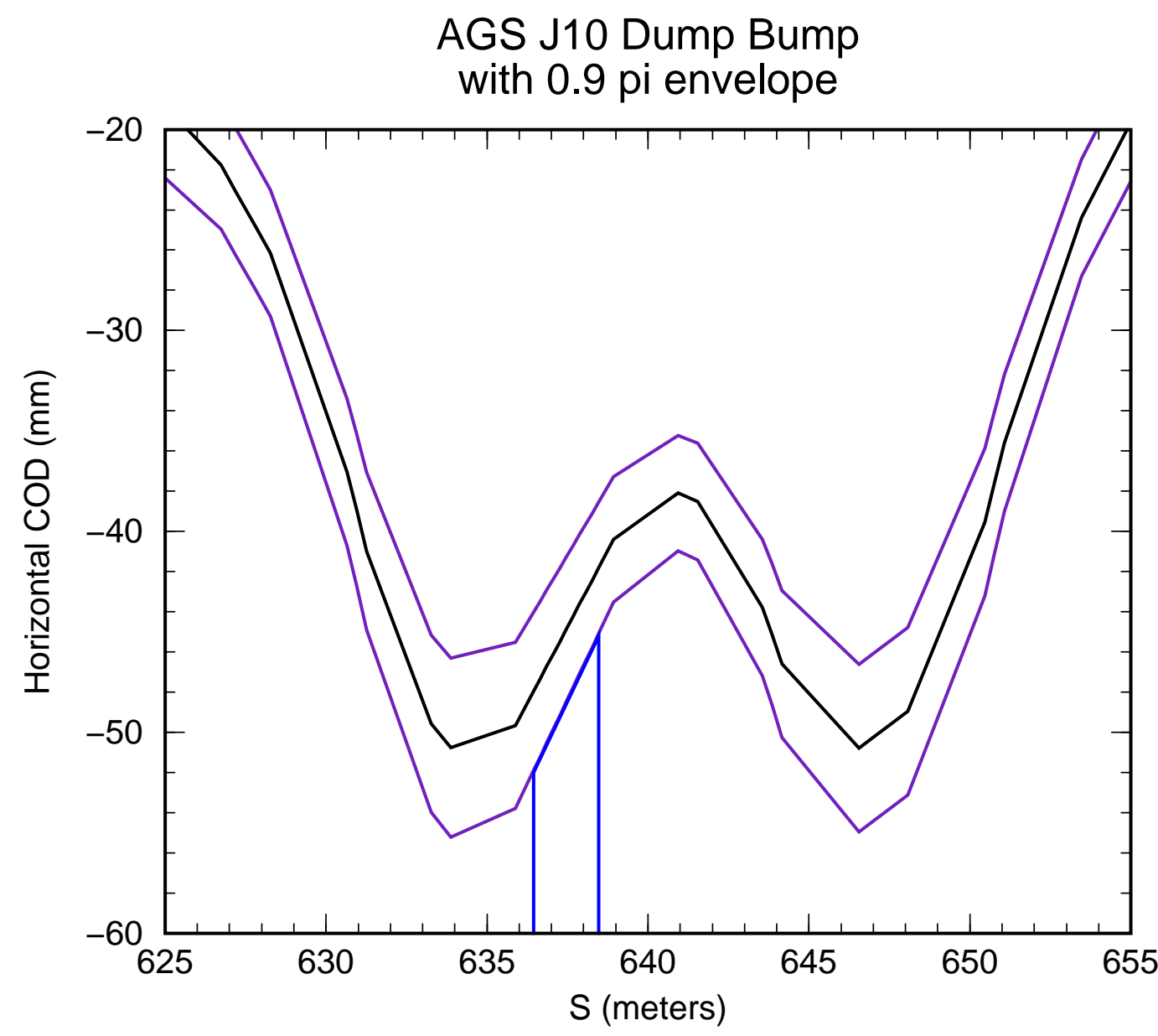

Figure 3: This is a "zoomed in" view of Figure 2. Here again the black curve is the J10 dump bump and the blue lines show the outline of the dump. The violet curves show the envelope of a $0.9 \pi$ emittance beam. This is the actual size of gold beam in AGS at extraction assuming a normalized emittance of $10 \pi$. Beam is put into the dump by increasing the amplitude of the bump. The downstream end of the dump may be moved further to the inside (more negative) than shown to ensure that beam hits the upstream end. The circulating beam direction here is from left to right. 


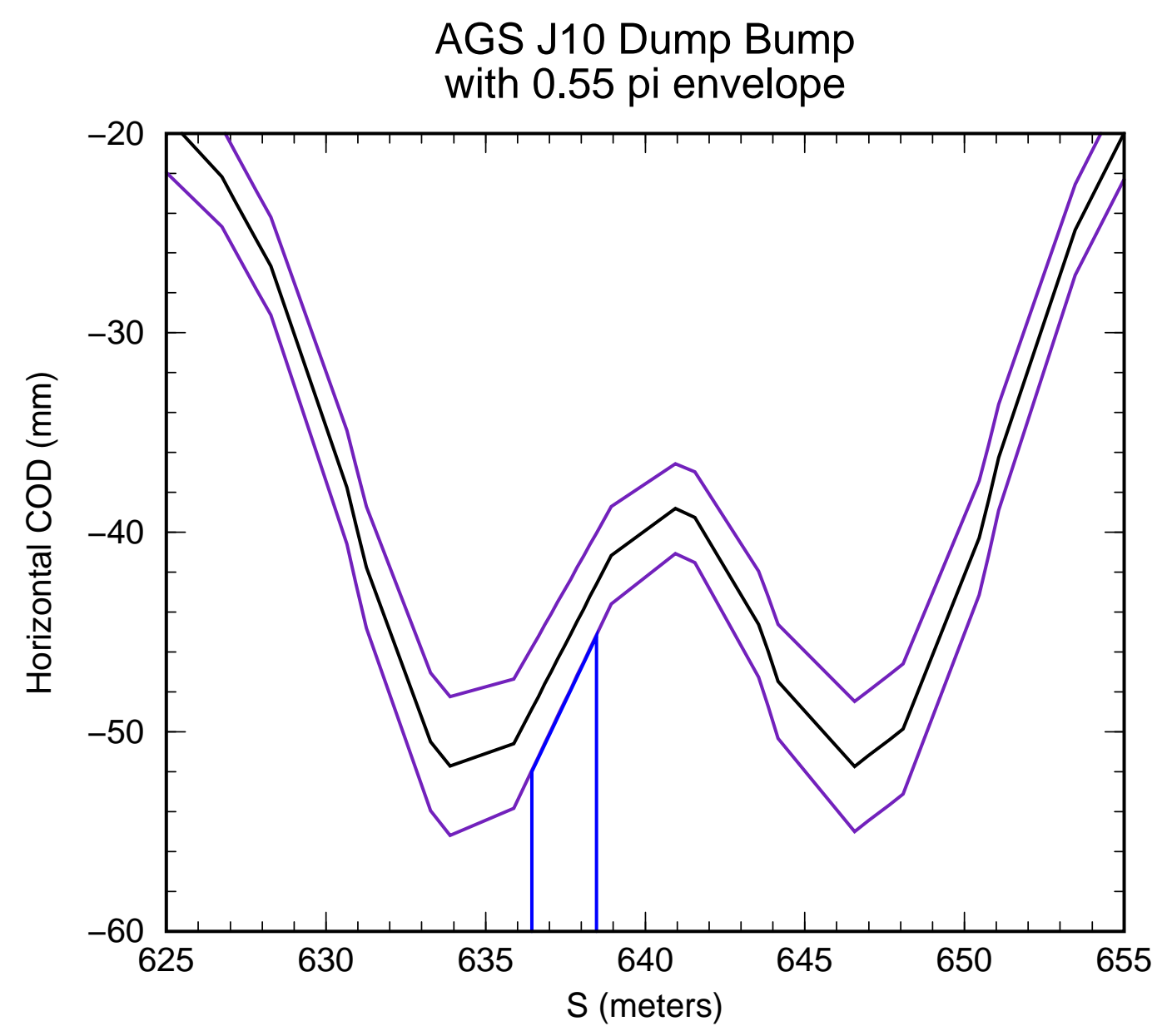

Figure 4: Here the bump amplitude has been inreased causing beam loss on the dump and reducing the beam emittance to $0.55 \pi$. 


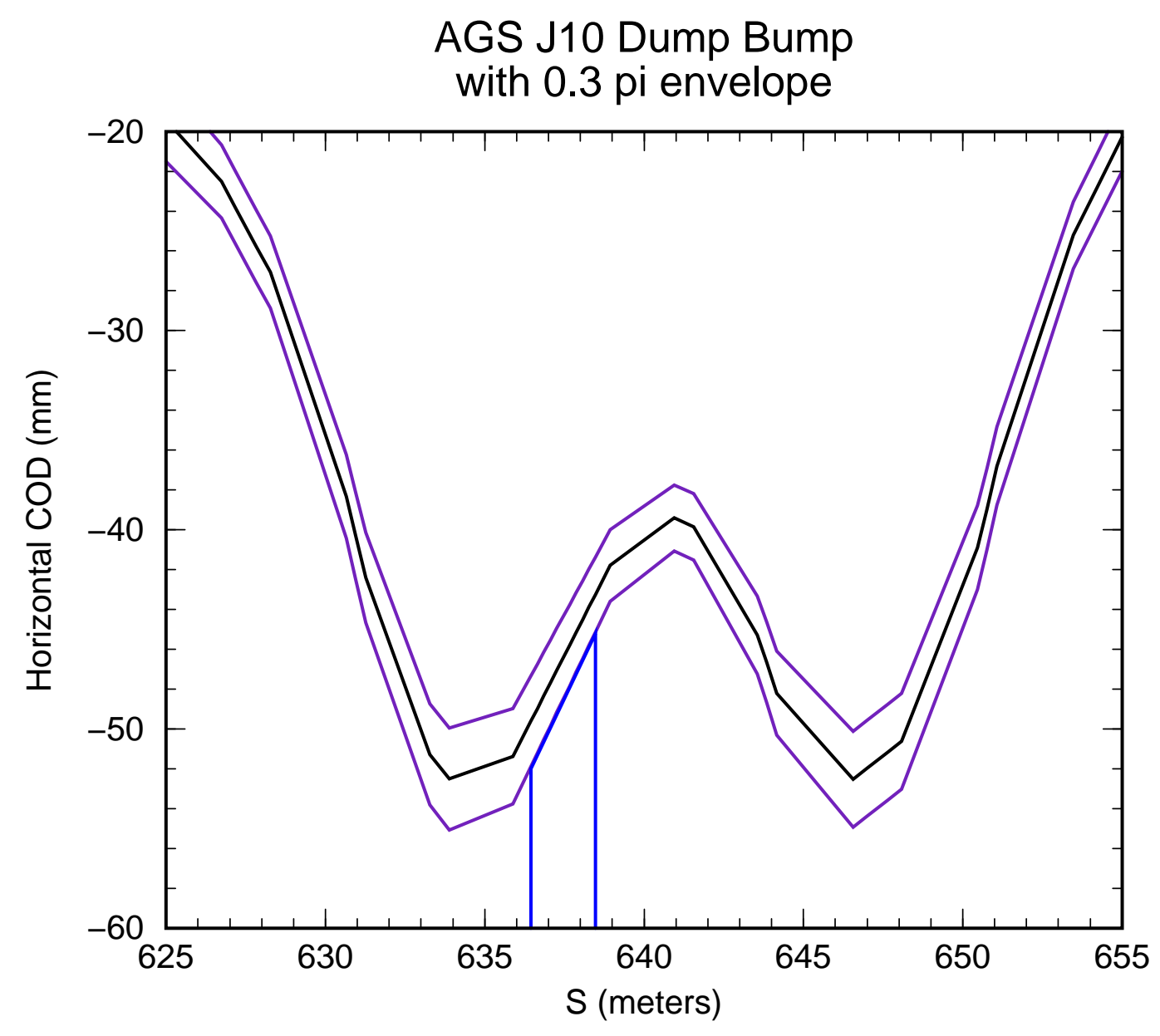

Figure 5: Here the bump amplitude has been inreased further, again causing beam loss on the dump and reducing the beam emittance to $0.3 \pi$. 


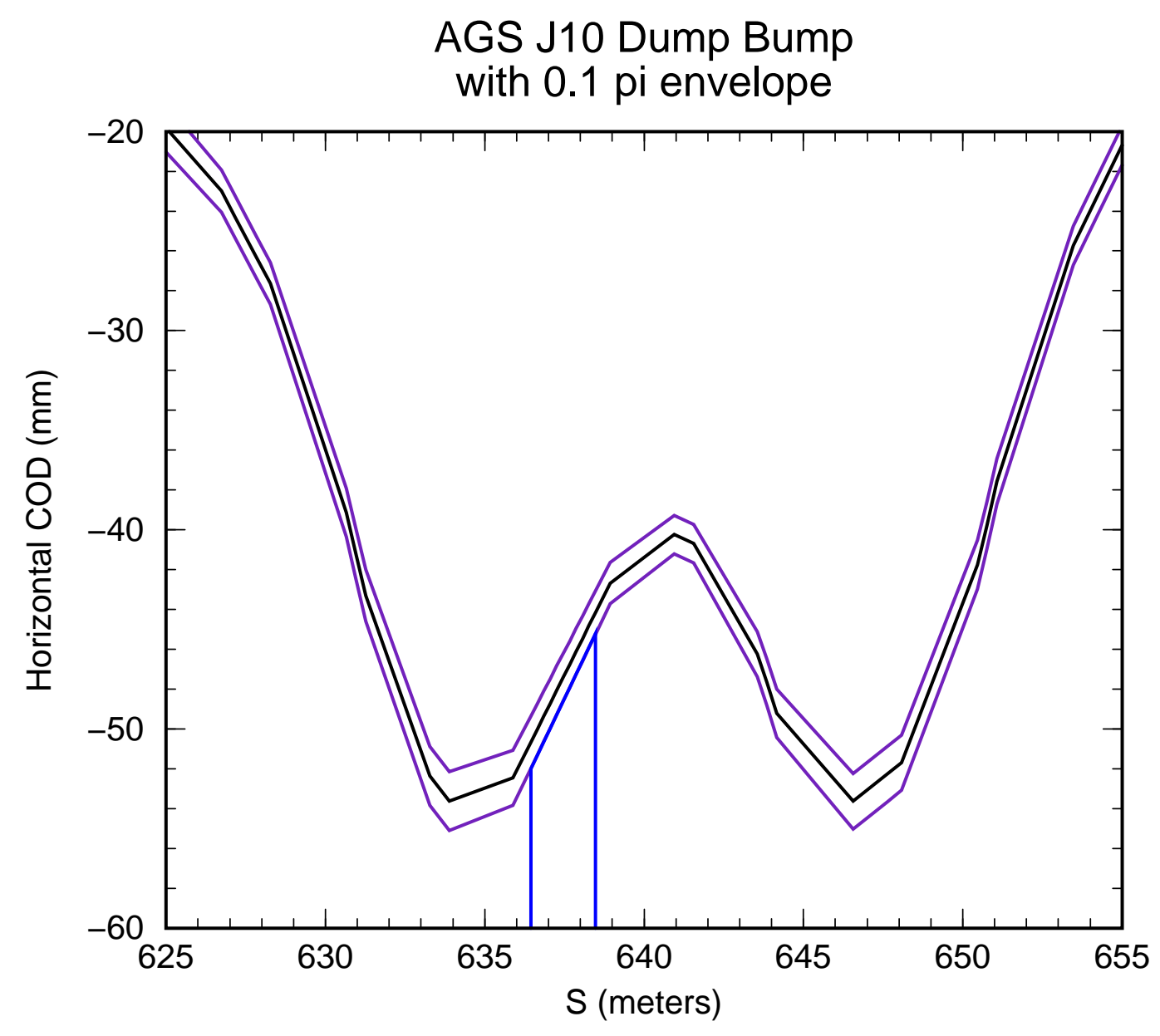

Figure 6: Here the bump amplitude has been inreased further still, causing beam loss on the dump and reducing the beam emittance to $0.1 \pi$. 


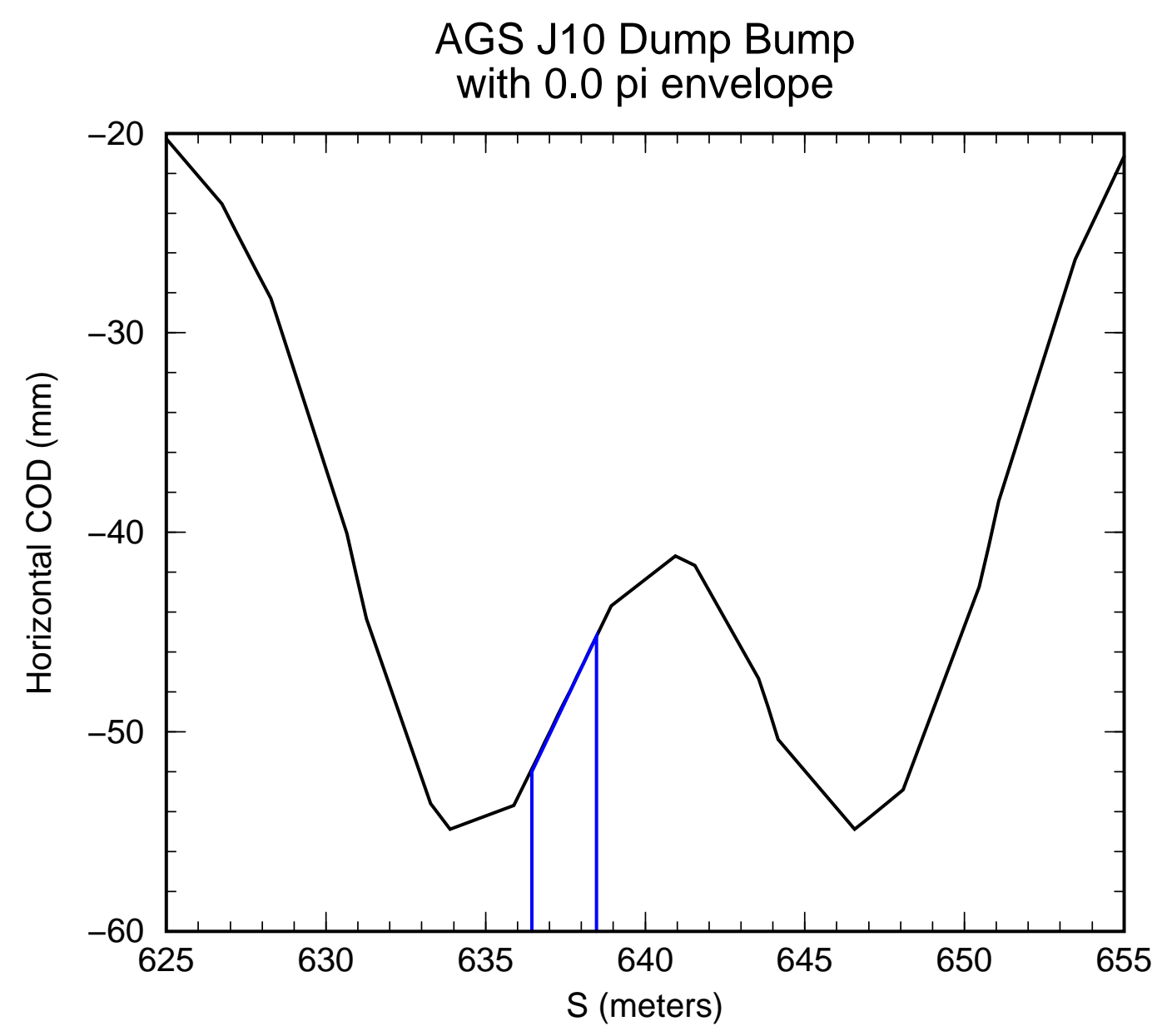

Figure 7: Here the bump amplitude has been inreased further, causing beam loss on the dump and reducing the beam emittance to $0.0 \pi$. 


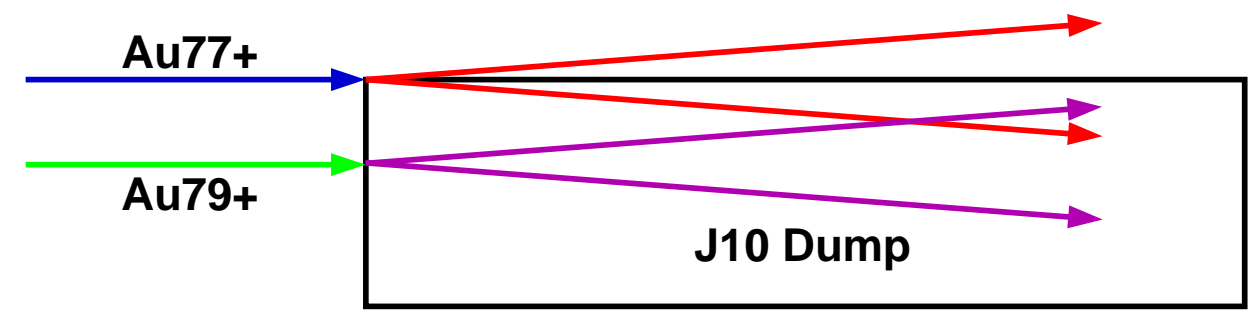

Figure 8: Here the blue and red arrows illustrate scattering of Au77+ ions at the surface of the dump. Some of the scattered particles continue downstream. This is the "standard" way of putting gold ions into the dump. The green and purple arrows illustrate the result of putting Au79+ ions into the upstream face of the dump. In this case the scattered particles are absorbed by the dump. 


\section{Stripping to Au79+ on the Dump}

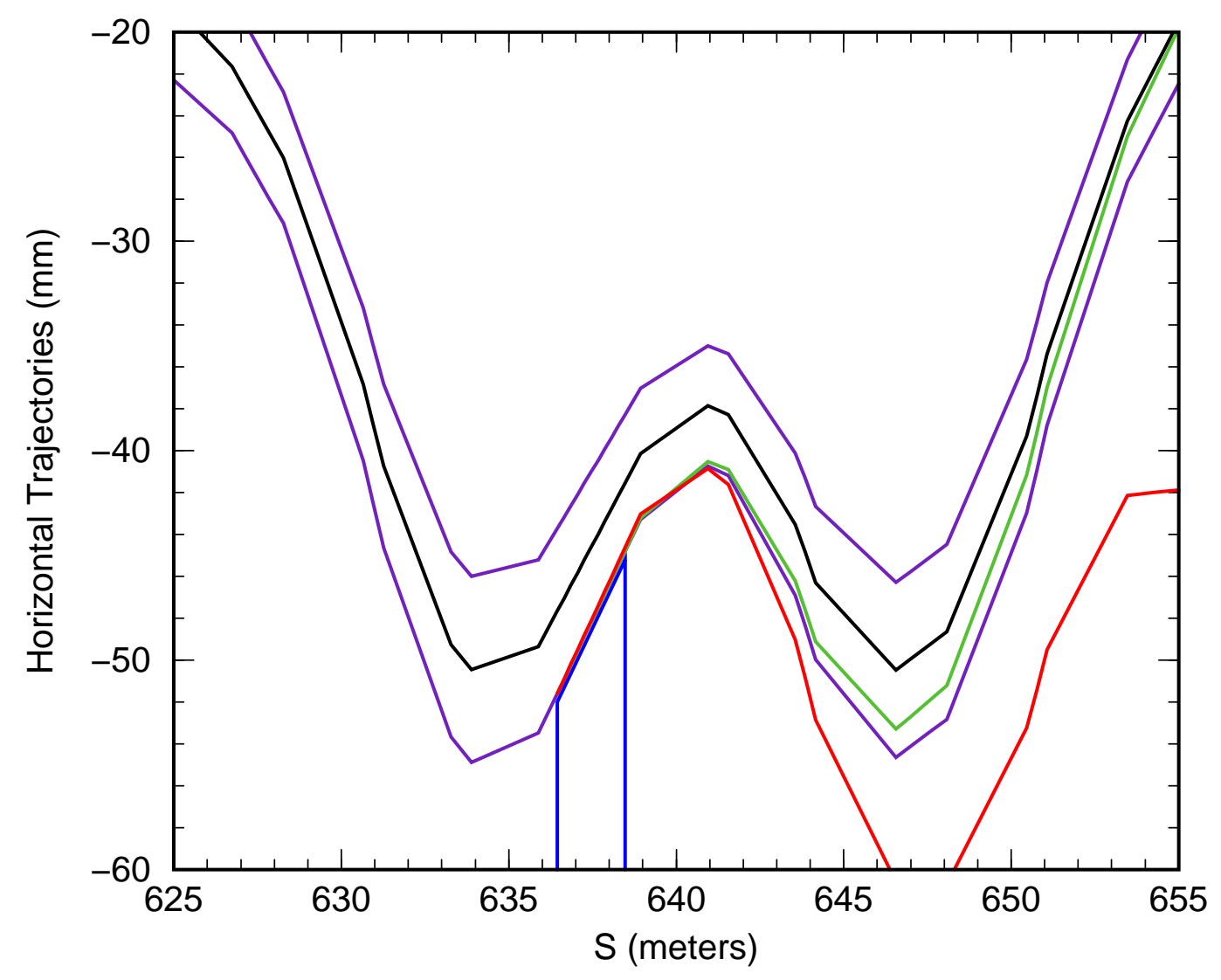

Figure 9: Here the red curve is the trajectory of a gold ion that has been stripped to Au79+ by just grazing the surface of the dump. The green curve is the trajectory of an unstripped Au77+ ion with the same initial coordinates. As before, the black curve is the dump bump, the violet curves show the envelope of the $0.9 \pi$ emittance beam and the blue lines show the outline of the dump. 


\section{Stripping to Au79+ on the Dump}

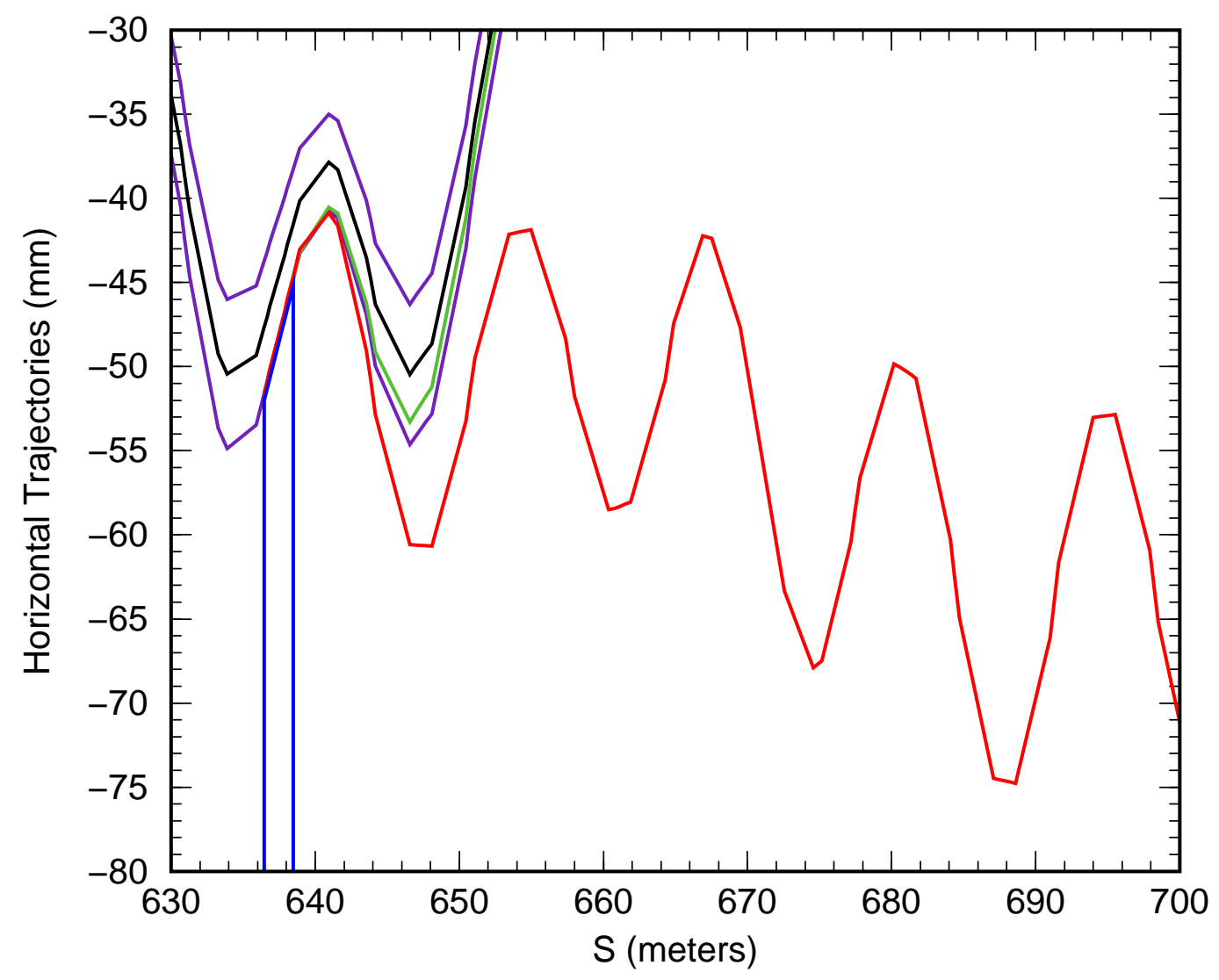

Figure 10: This a "zoomed out" view of Figure 9 showing the trajectory of the stripped Au79+ ion. Going from left to right, the local minima of the trajectory are located in the $\mathrm{J} 13, \mathrm{~J} 17, \mathrm{~K} 1$, and $\mathrm{K} 5$ straights respectively. During Run 8 high loss was observed at J17 before the dump position was optimized and before the dump bump was strengthened. 


\section{Stripping to Au79+ on the Dump}

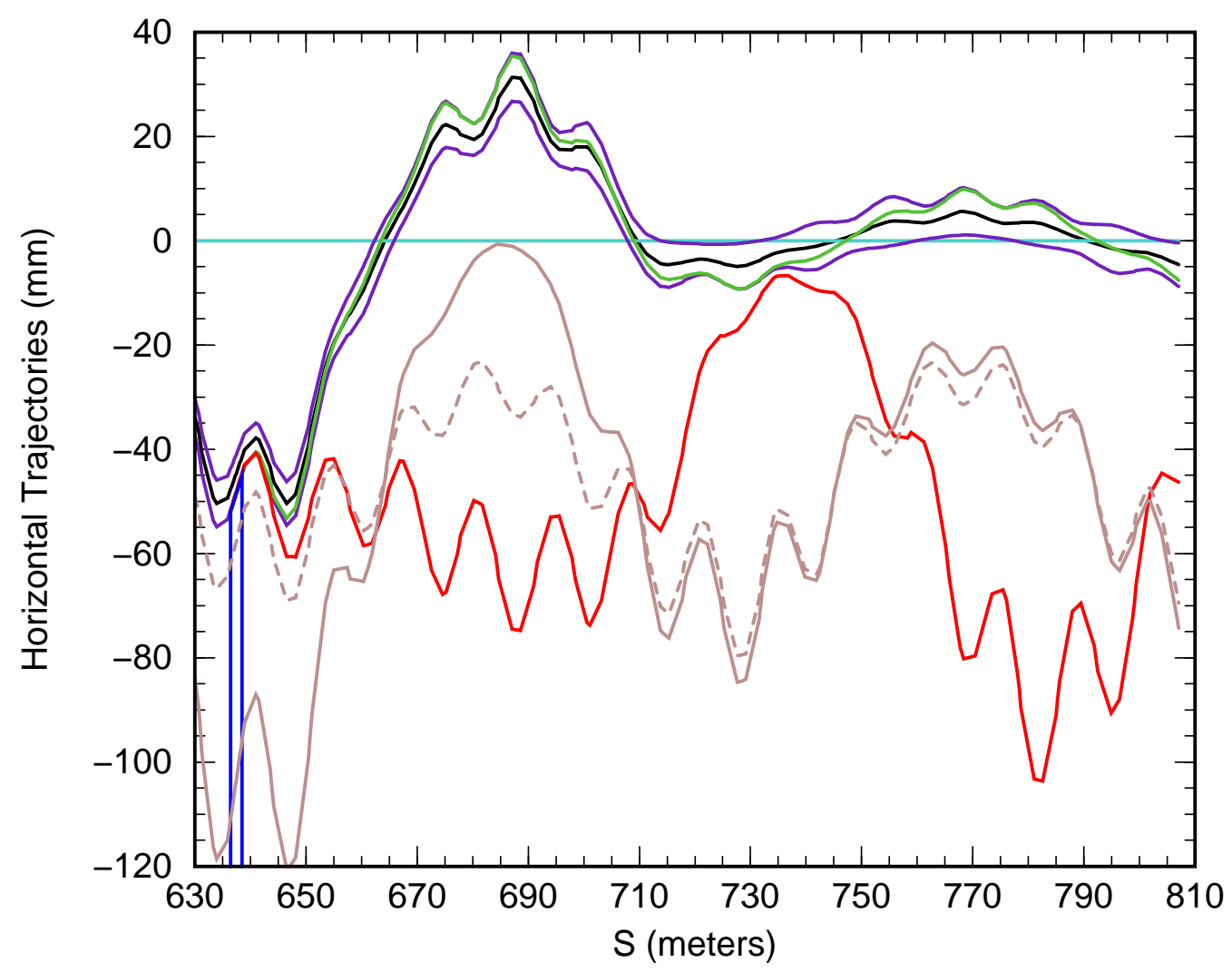

Figure 11: Here we have "zoomed out" further. The solid brown curve is the equilibrium orbit about which the Au79+ ion is oscillating. The dashed curve is the periodic dispersion multiplied by $\Delta p / p=-2 / 77$. The minimum of the Au79+ trajectory near $s=782 \mathrm{~m}$ is in the L13 straight. During Run 10 beam loss was observed at L9 when unstripped beam (Au77+) was put into the dump. 


\section{Stripping to Au78+ on the Dump}

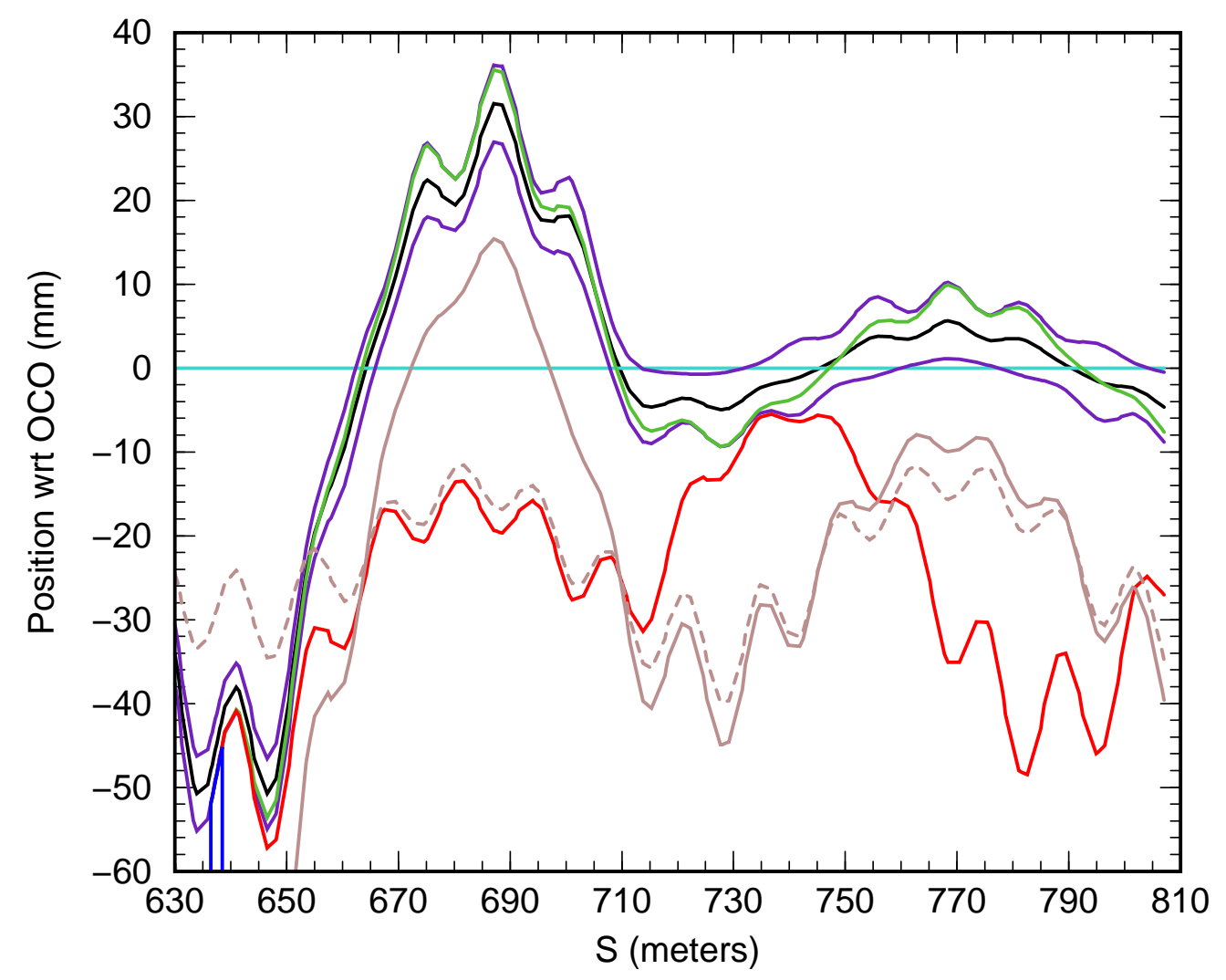

Figure 12: Here the red curve is the trajectory of a gold ion that has been stripped to Au78+ by just grazing the surface of the dump. The solid brown curve is the equilibrium orbit about which the Au78+ ion is oscillating. The dashed curve is the periodic dispersion multiplied by $\Delta p / p=-1 / 77$. The minimum of the Au78+ trajectory near $s=782 \mathrm{~m}$ is in the L13 straight. 


\section{Stripping to Au79+ in J7 Straight}

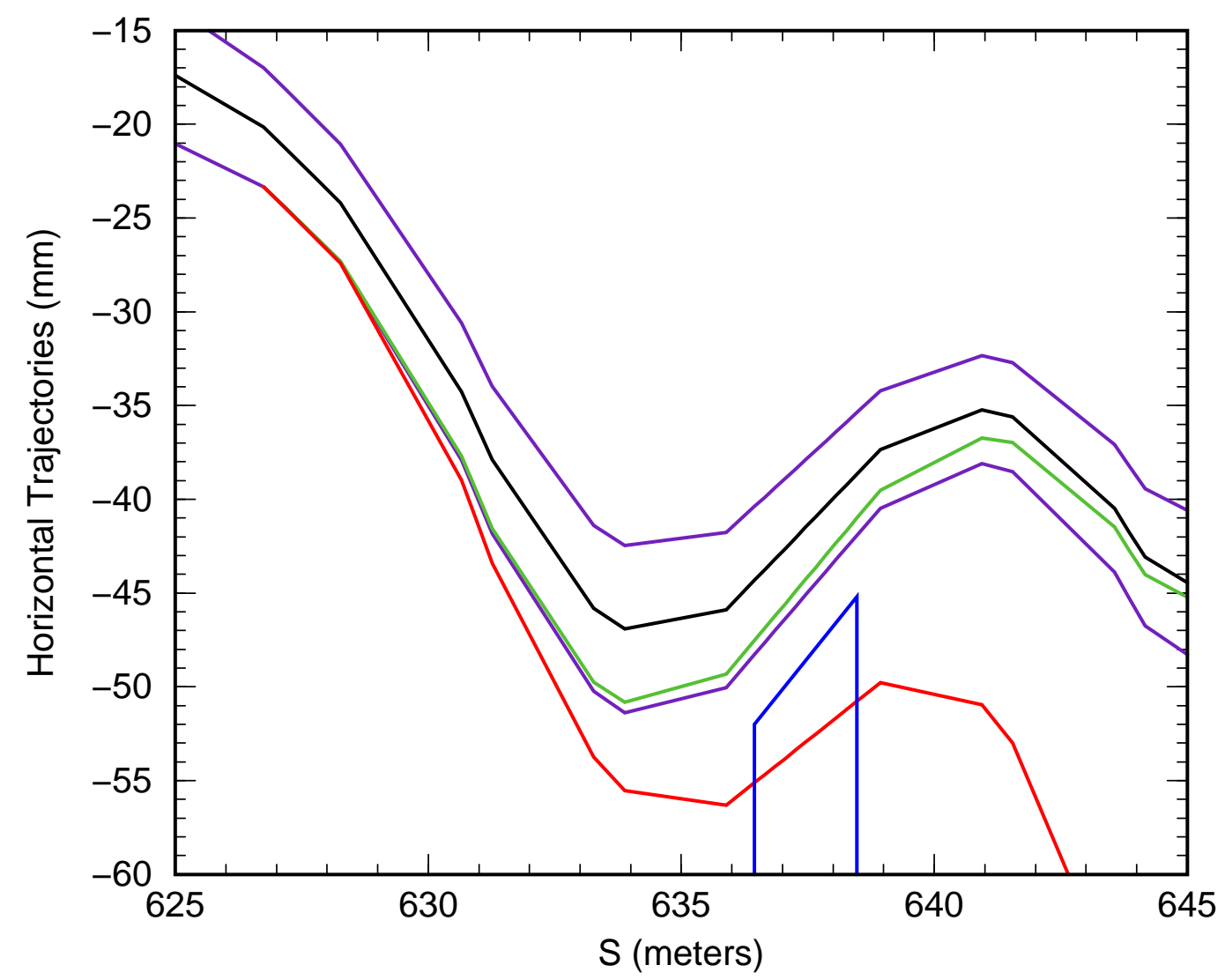

Figure 13: Here the red curve shows the trajectory of a gold ion that has been stripped to Au79+ by passing through a stripper located in the J7 straight. The green curve is the trajectory of an unstripped Au77+ ion with the same initial coordinates. As before, the black curve is the dump bump, the violet curves show the envelope of the $0.9 \pi$ emittance beam, and the blue lines show the outline of the dump. The stripped ion is lost on the upstream face of the dump. 


\section{Stripping to Au79+ in J7 Straight}

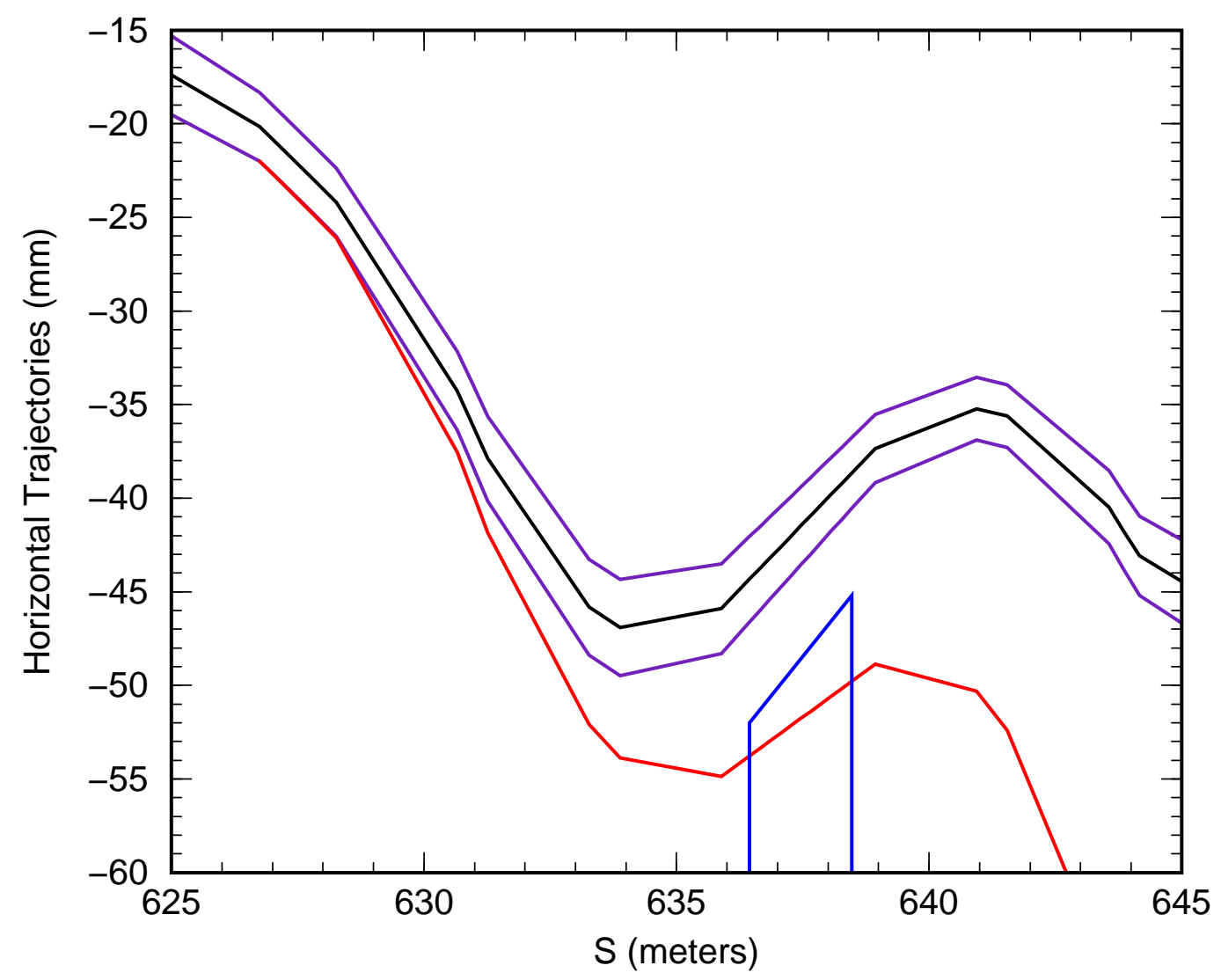

Figure 14: Holding the dump bump fixed the J7 stripper is plunged further into the beam thereby reducing the circulating beam emittance while putting the stripped beam into the upstream face of the dump. 


\section{Stripping to Au79+ in J7 Straight}

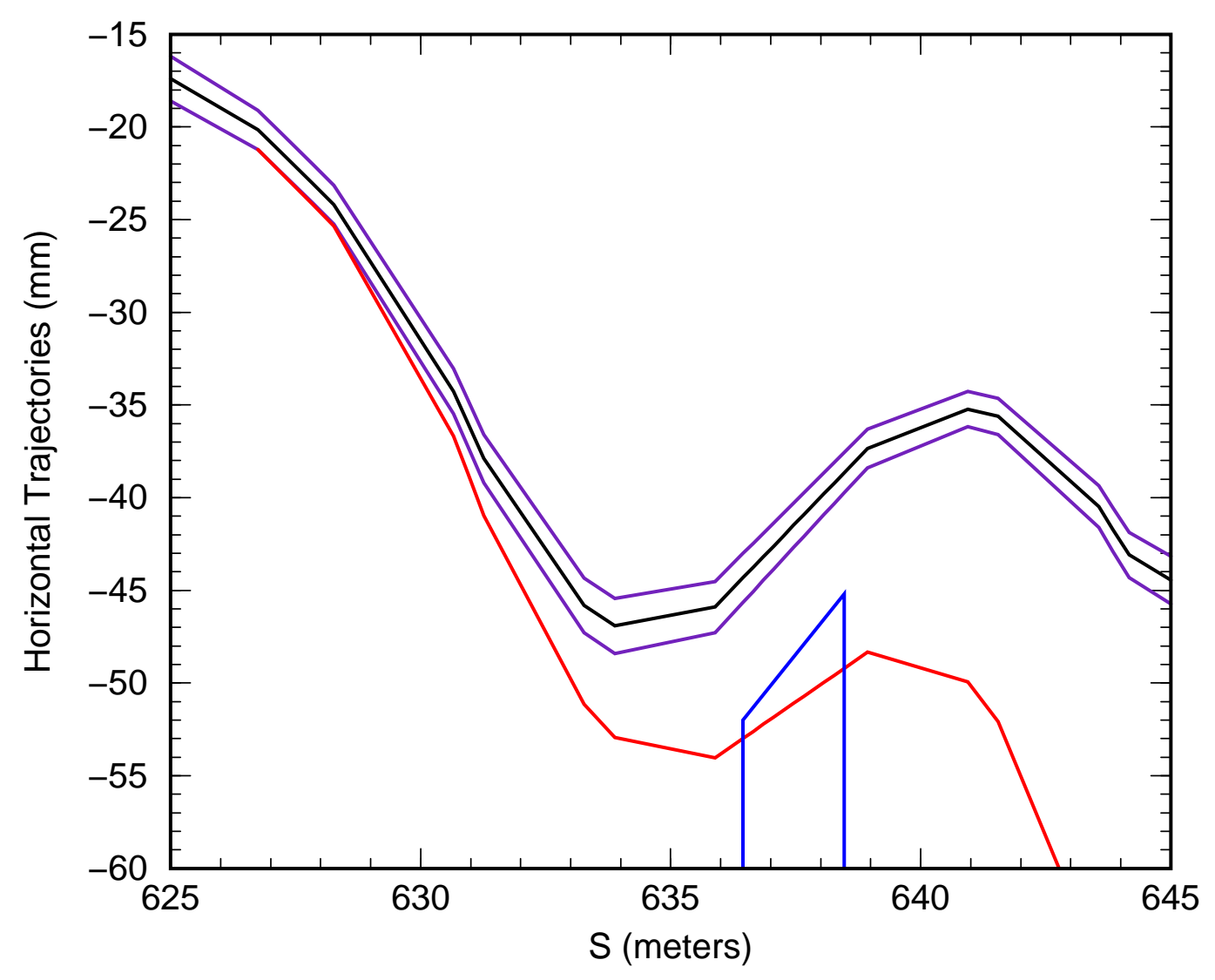

Figure 15: The J7 stripper is plunged still further into the beam. The circulating beam emittance is reduced further while the stripped beam continues to be put into the upstream face of the dump. 


\section{Stripping to Au79+ in J7 straight}

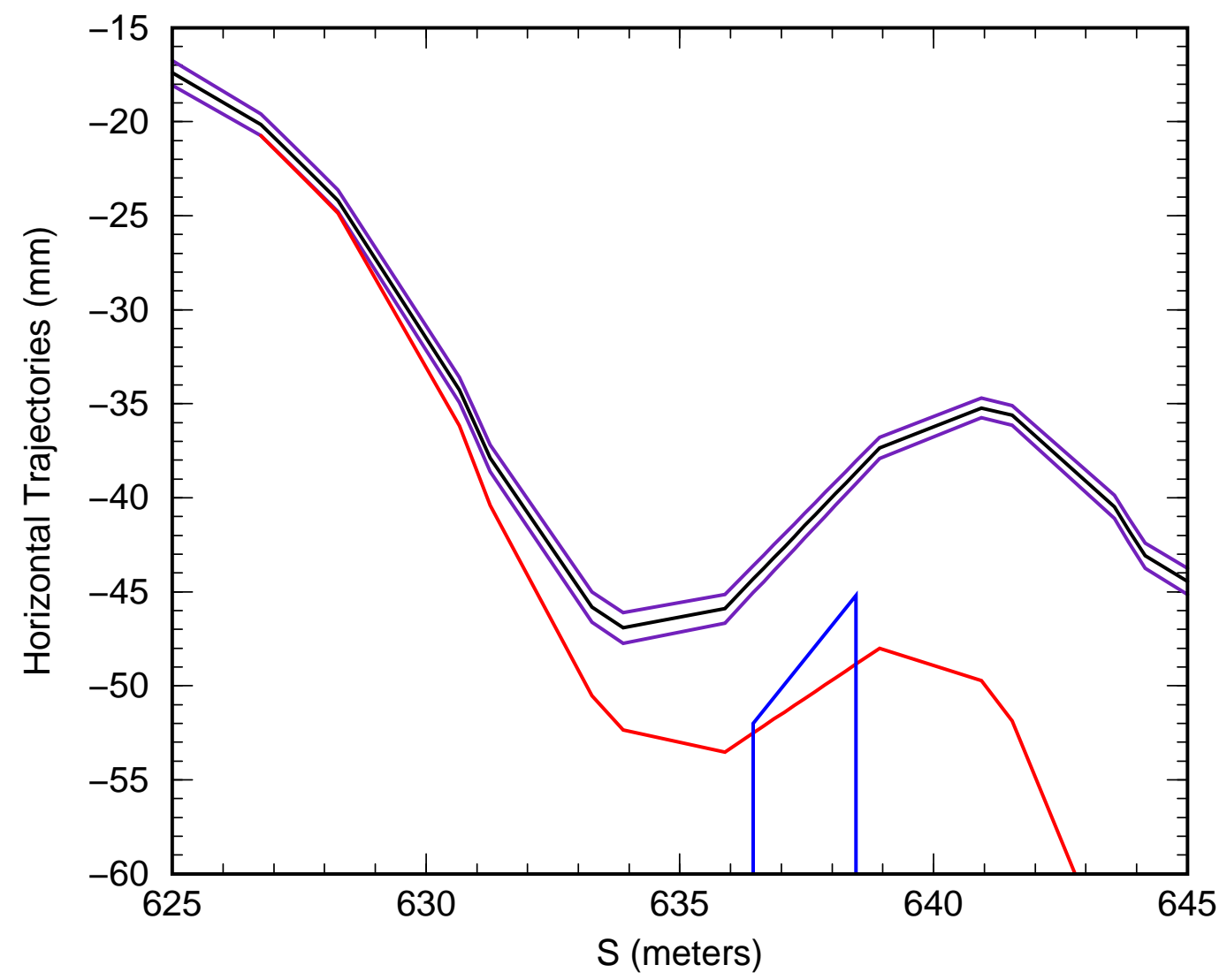

Figure 16: The J7 stripper is plunged further into the beam. The circulating beam emittance is reduced further while the stripped beam continues to be put into the upstream face of the dump. 


\section{Stripping to Au79+ in J7 Straight}

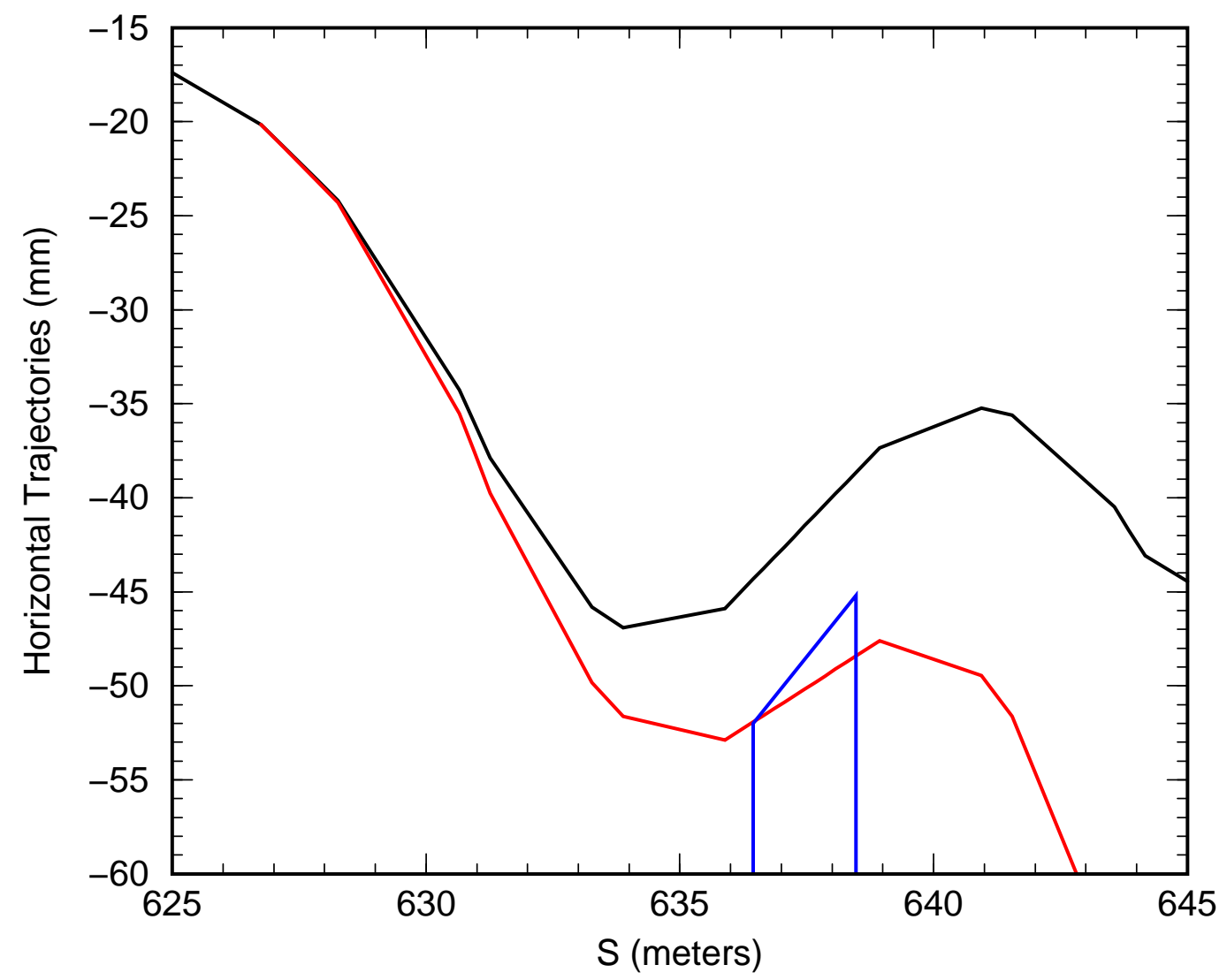

Figure 17: The J7 stripper is plunged into the the last bit of circulating beam. The stripped beam is put into the upstream face of the dump. 


\section{Stripping to Au79+ in J7 Straight}

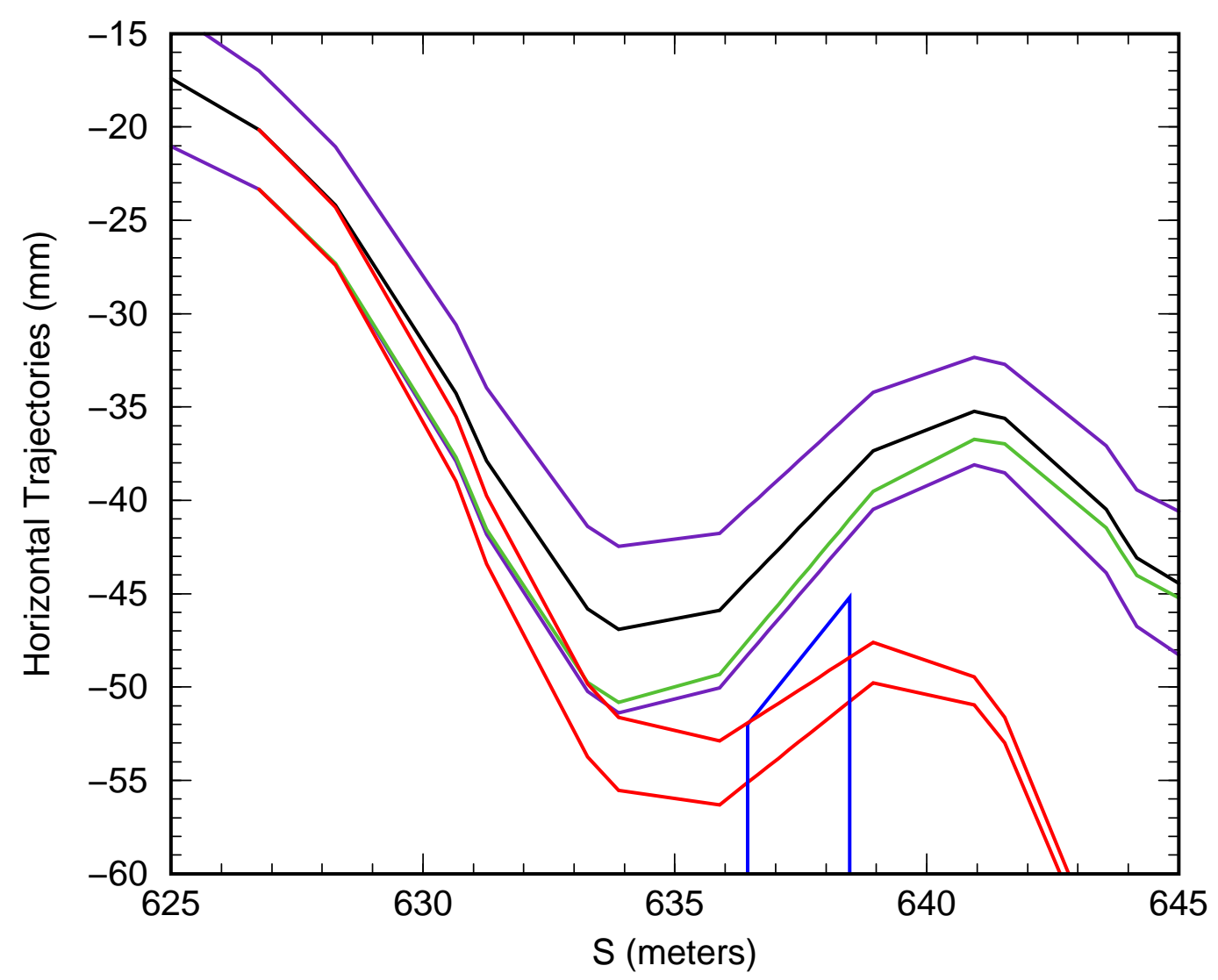

Figure 18: Here are the initial and final Au79+ ion trajectories obtained as the J7 stripper is plunged into the circulating beam. Note that the ions are lost at different locations on the face of the dump as the stripper moves into the beam. Note also that no beam hits the magnet vacuum chamber wall which sits at -3.406 inches $(-86.5 \mathrm{~mm})$. 
Stripping to Au79+ in J7 Straight

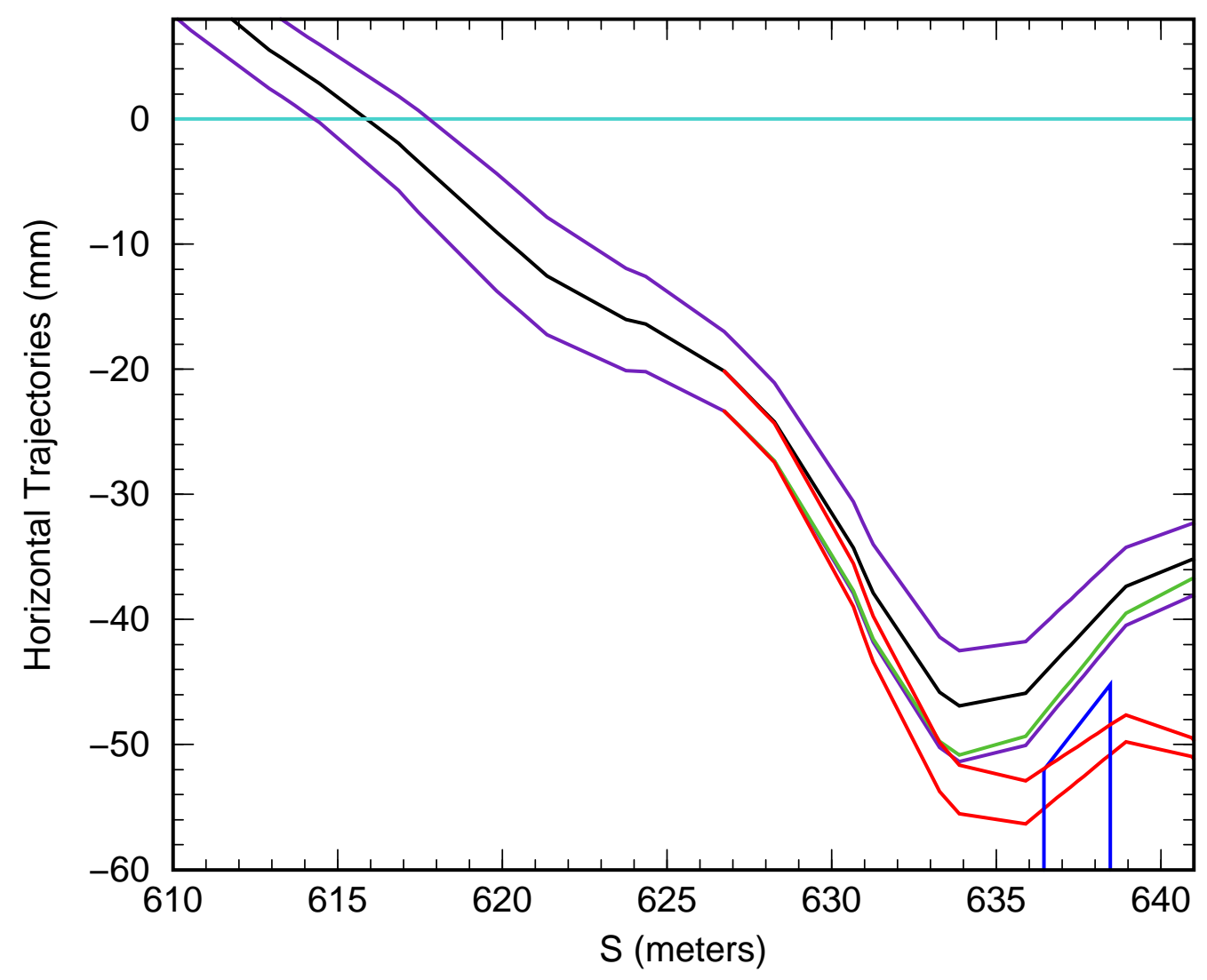

Figure 19: Here is a "zoomed out" view of the initial and final trajectories obtained as the J7 stripper is plunged into the circulating beam. 
Stripping to Au79+ in J5 Straight

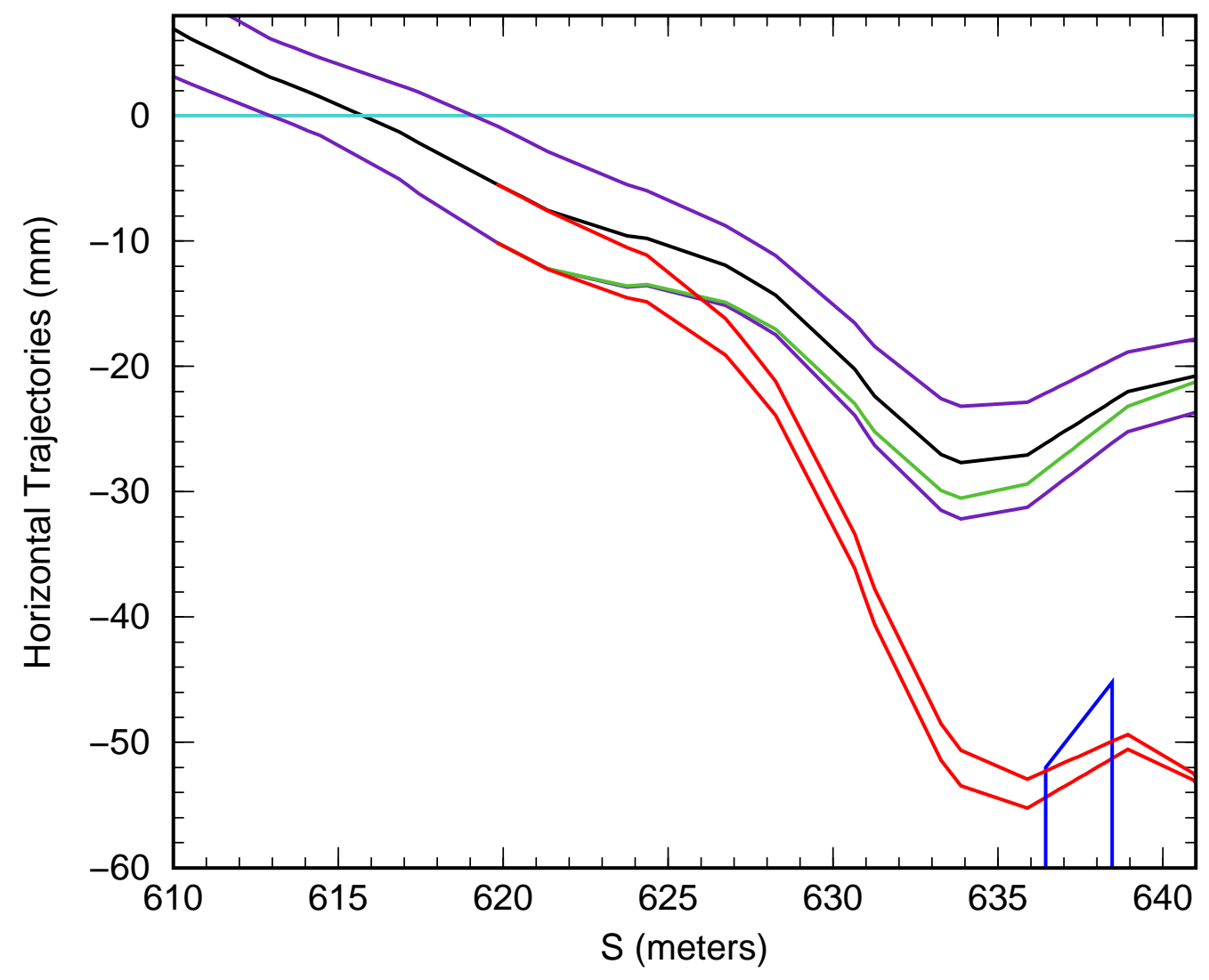

Figure 20: Here are the initial and final Au79+ trajectories obtained for the case in which a stripper is plunged into the circulating beam in the J5 straight. 


\section{Stripping to Au79+ in J3 Straight}

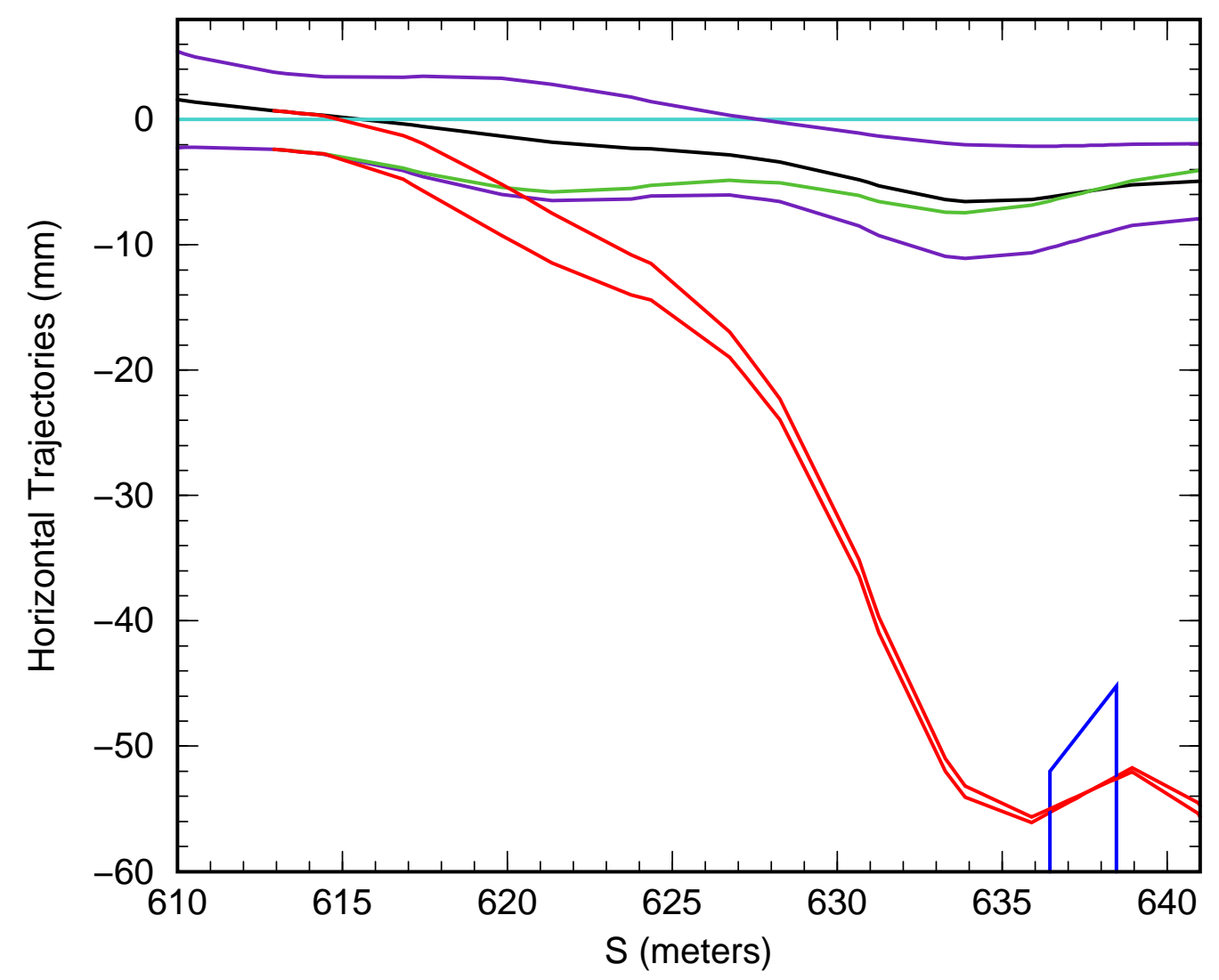

Figure 21: Here are the initial and final Au79+ trajectories obtained for the case in which a stripper is plunged into the circulating beam in the J3 straight. Note that because the phase advance between J3 and J10 is close to $\pi / 2$, the trajectories converge to a point on the upstream face of the dump. 


\section{Stripping to Au79+ in J1 Straight}

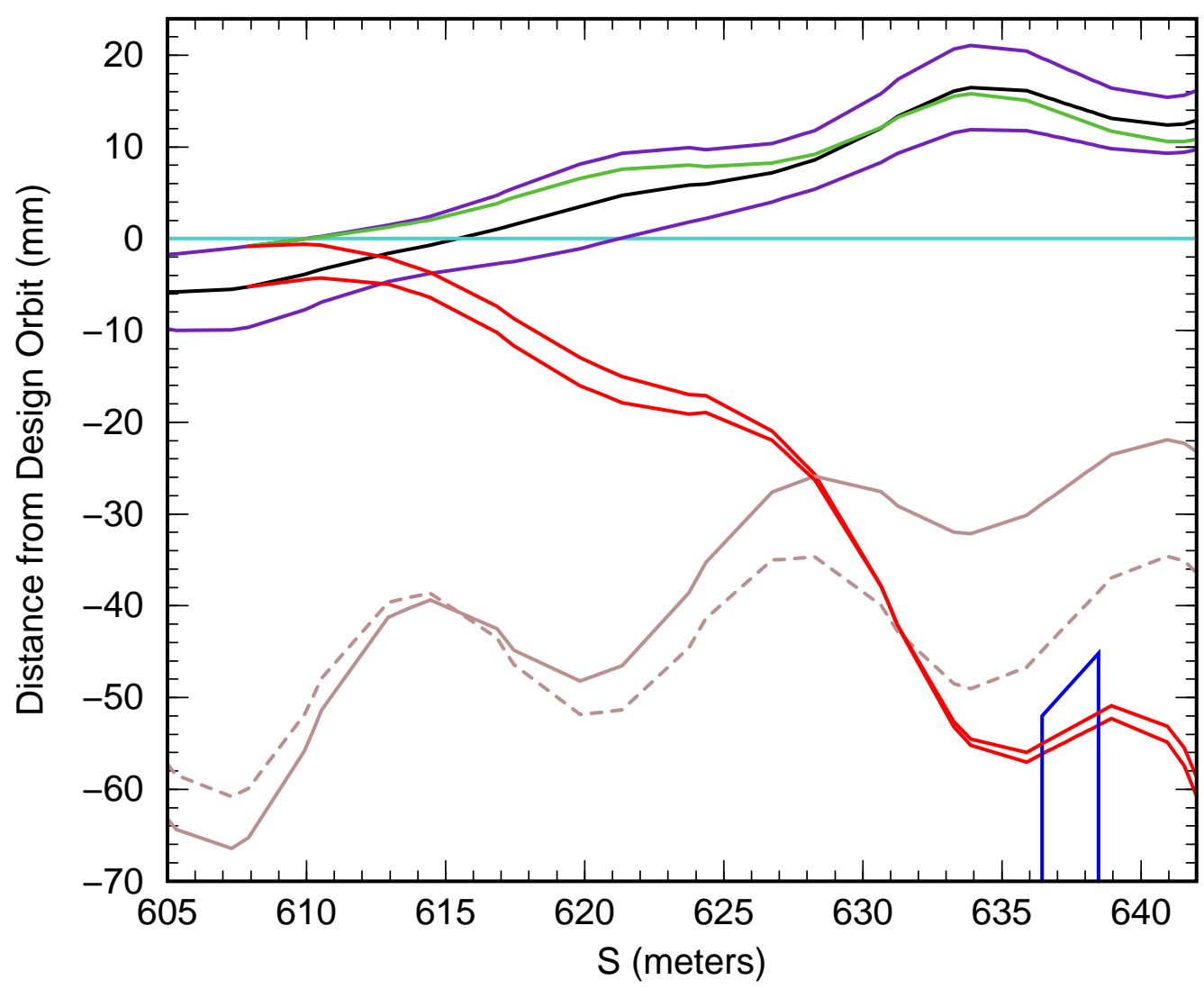

Figure 22: Here are the initial and final Au79+ trajectories obtained for the case in which a stripper is plunged into the circulating beam (now from the outside) in the J1 straight. Note that because the phase advance between $\mathrm{J} 1$ and $\mathrm{J} 10$ is still close to $\pi / 2$, the trajectories are close to one another on the upstream face of the dump. 
Stripping to Au79+ in J1 Straight

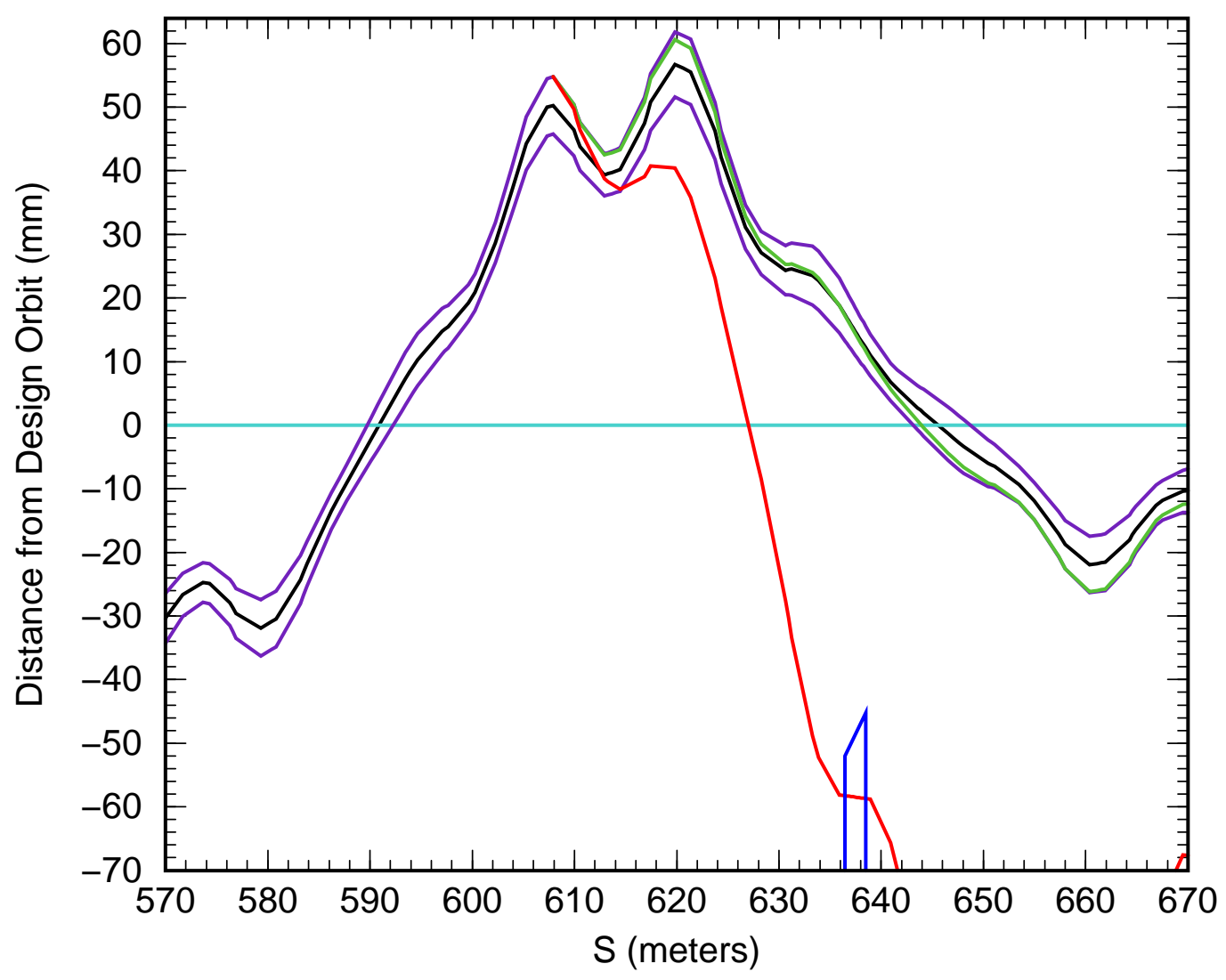

Figure 23: Here is the Au79+ trajectory obtained for the case in which a stripper is held fixed in the J1 straight while the circulating beam is moved into it with a programmable bump. 
Stripping to Au79+ in J1 Straight

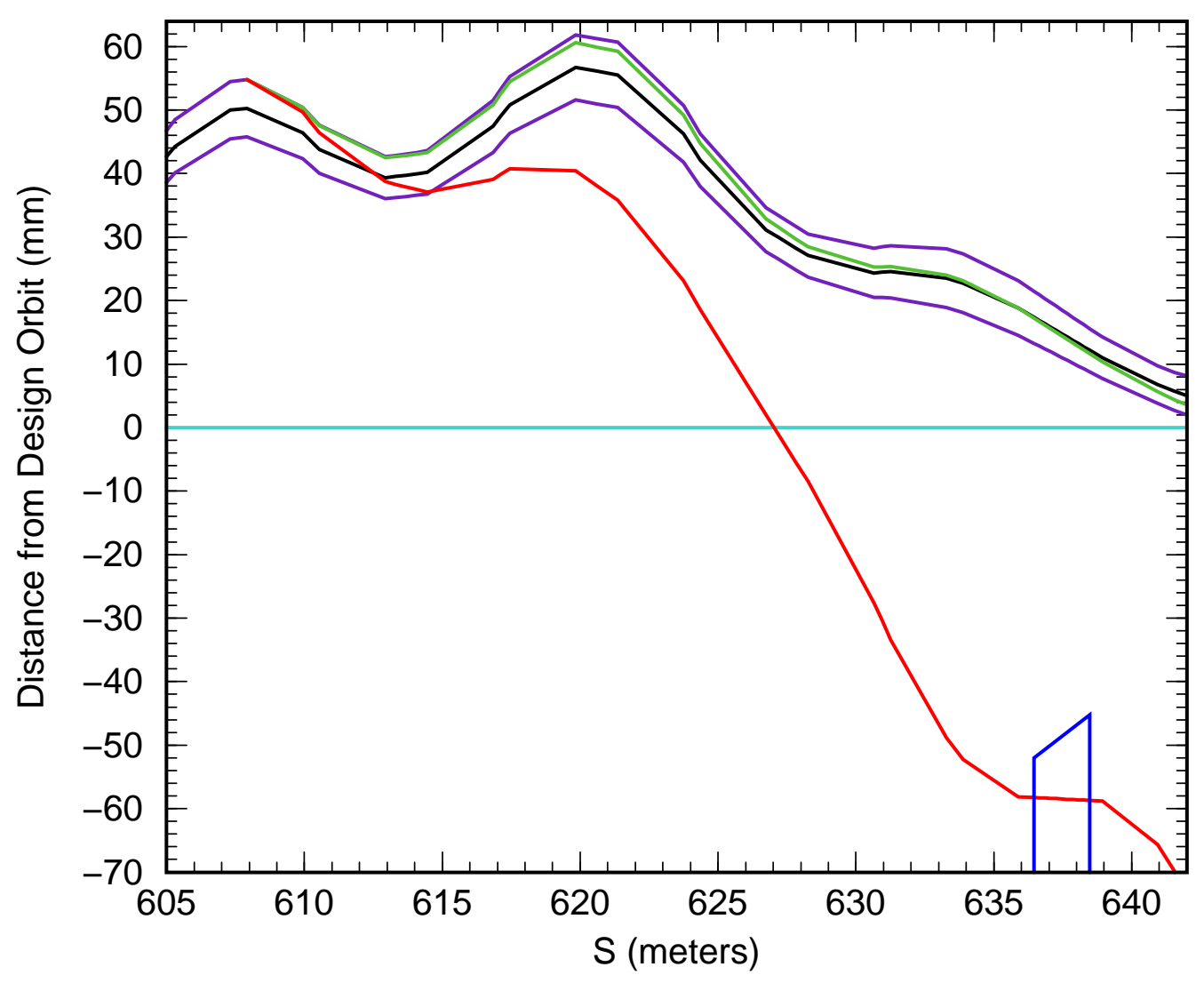

Figure 24: "Zoomed in" view of Figure 23. 


\section{Stripping to Au79+ in J1 Straight}

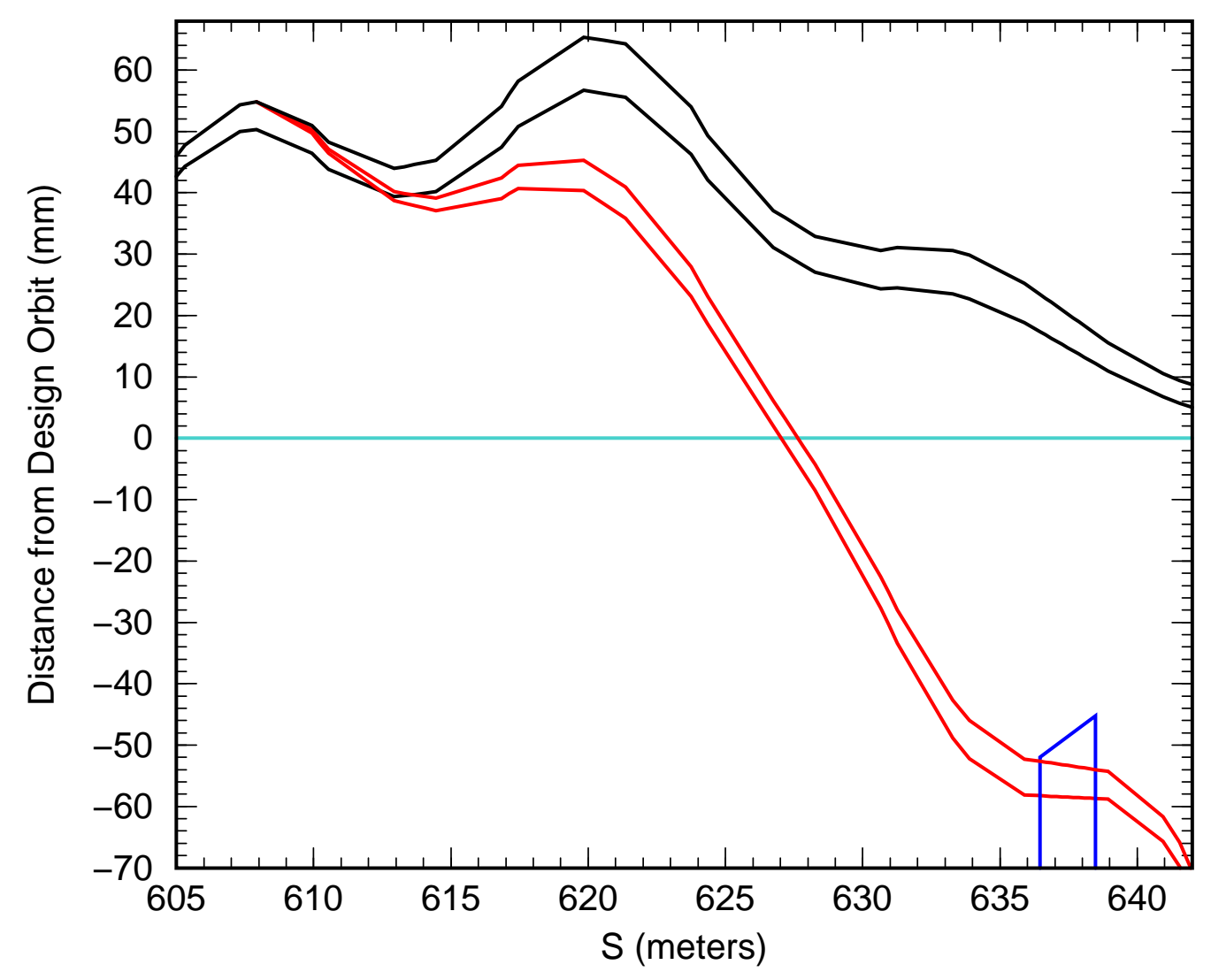

Figure 25: Closed orbit and Au79+ trajectory of Figure 24 and the Au79+ trajectory obtained with the orbit moved just to the point where all circulating beam has been removed. 


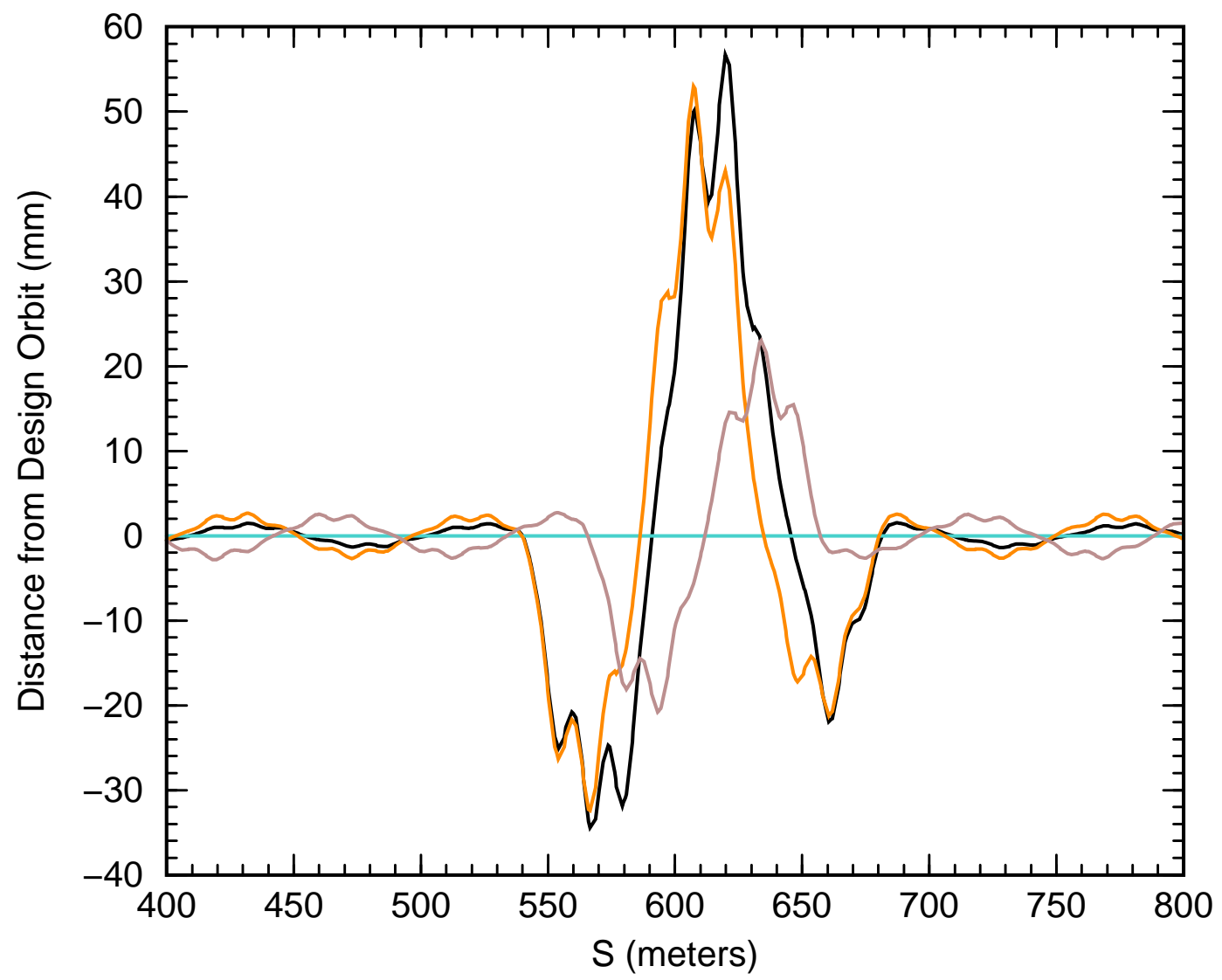

Figure 26: J1 Position and Angle Bumps (orange and brown curves) and their Superposition (black curve). 


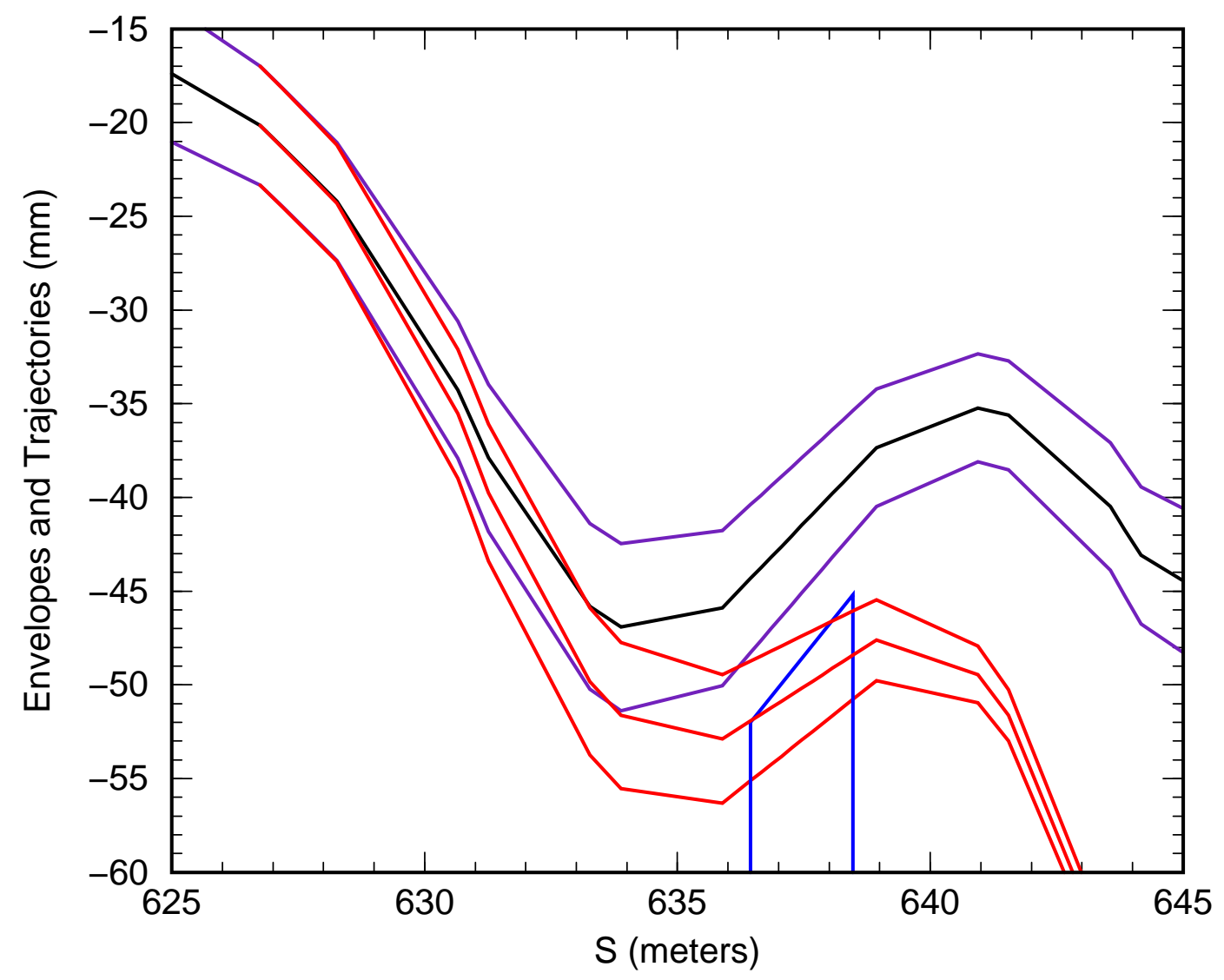

Figure 27: Here we see the Au79+ trajectories (red curves) resulting from plunging the $\mathrm{J} 7$ stripper from the inside and from the outside. The black curve is, as before, the dump bump and the violet curves show the envelope of the $0.9 \pi \mathrm{mm}$ mrad emittance circulating beam. In the plunging-frominside case, the Au79+ trajectories hit the upstream face of the dump. In the plunging-from-outside case, the Au79+ trajectories still hit the dump but they miss the upstream face. This may result in a dirtier dump with more debris downstream. 


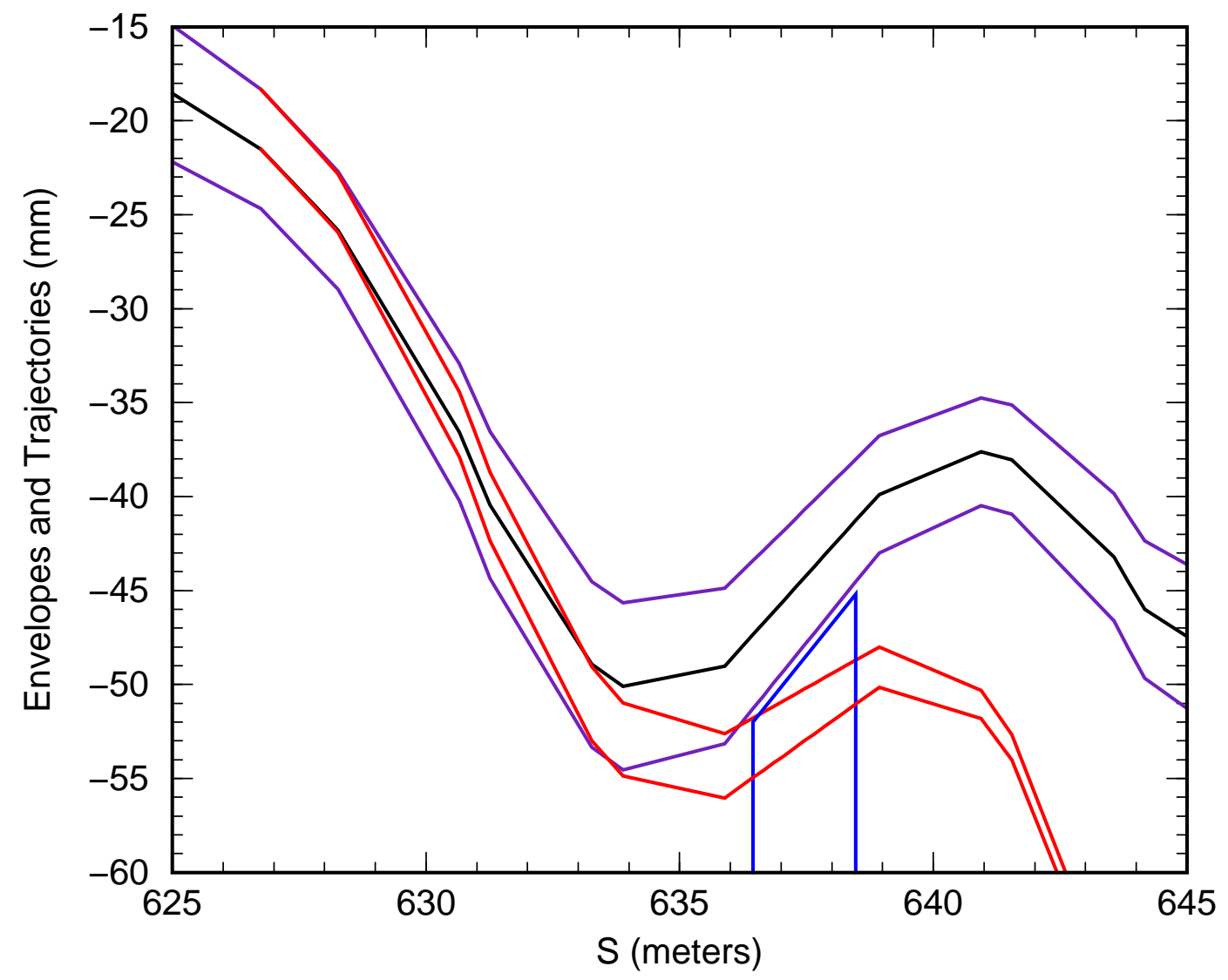

Figure 28: Here we see that we can make the plunging-from-outside trajectories hit the upstream face of the dump, but it requires bringing the circulating beam very close to the dump. This may make it difficult to set up the stripping in practice. 


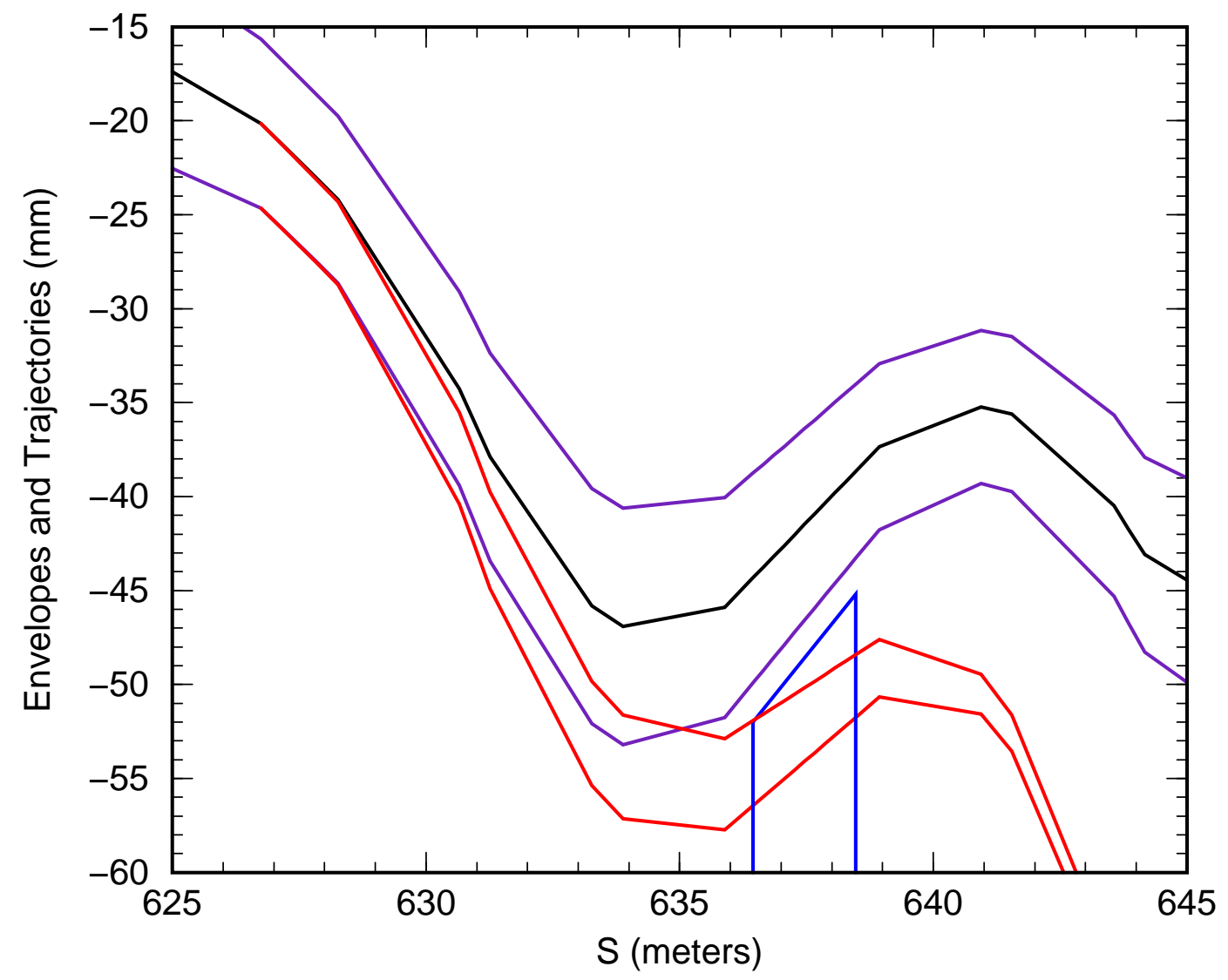

Figure 29: Stripping from the inside at J7 with twice the nominal circulating beam emittance $(2 \times 0.9 \pi=1.8 \pi)$. Here we see that there is still clearance between the dump and the circulating beam. 
Stripping to Au79+ and Au78+ in J7 Straight

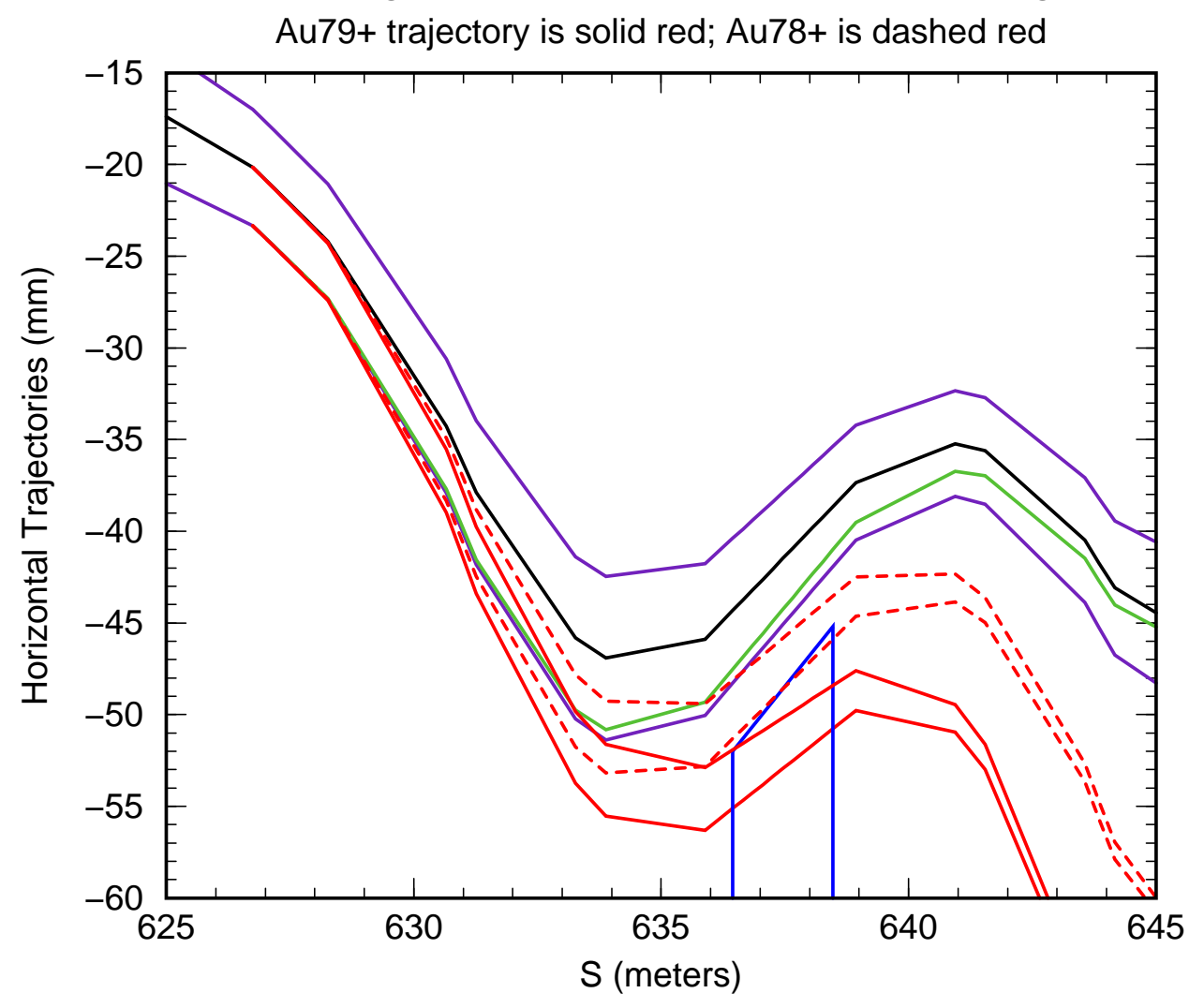

Figure 30: Stripping from Au77+ to Au79+ and Au78+ in the J7 straight. The solid and dashed red curves are the Au79+ and Au78+ trajectories respectively. Circulating beam emittance is $0.9 \pi \mathrm{mm} \mathrm{mrad}$. 


\section{Trajectory of Au78+ Produced in J7 Straight}

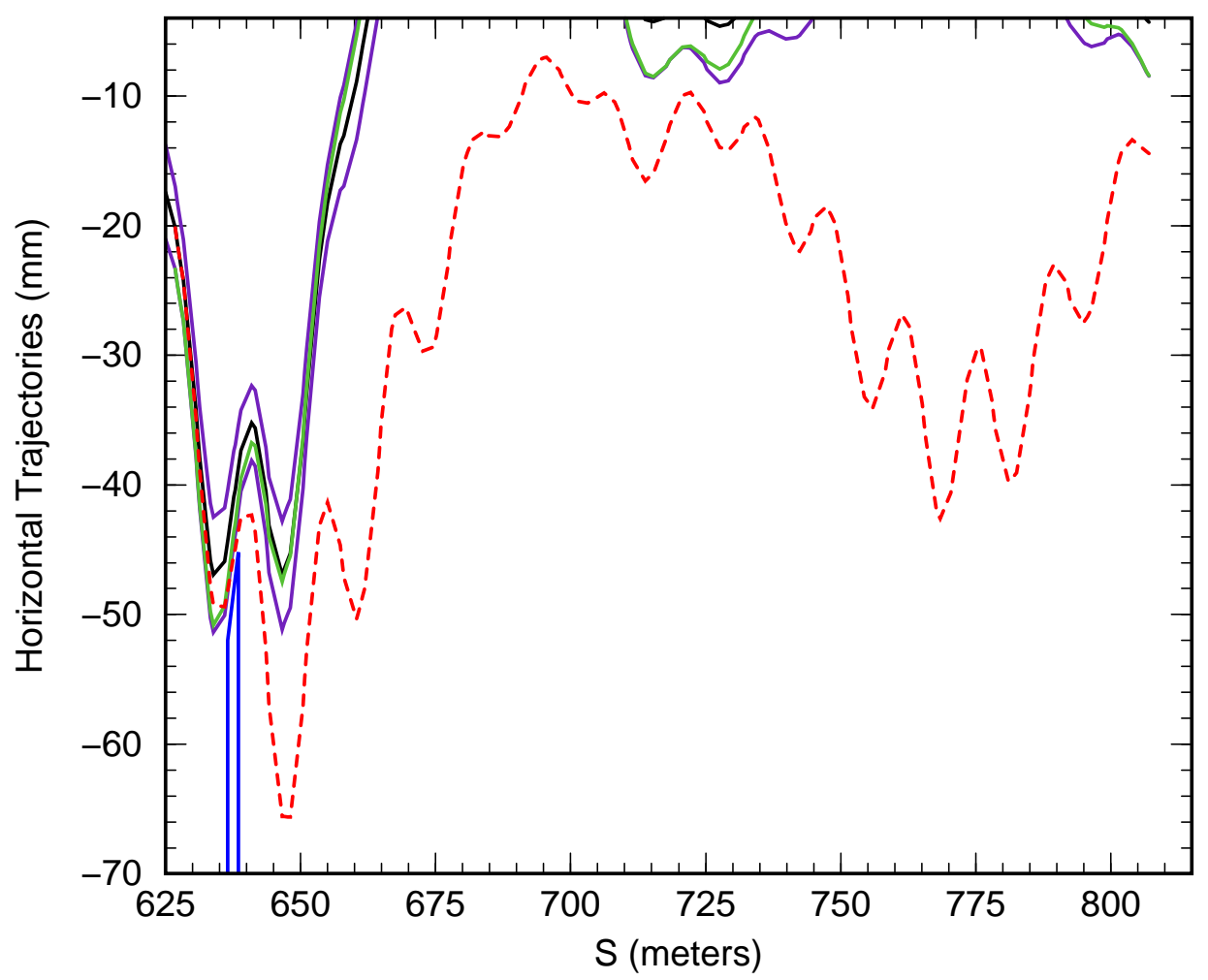

Figure 31: This is a "zoomed out" view of Figure 30 showing the Au78+ trajectory that survives beyond the J10 dump. The minima of trajectory near 647 and $768 \mathrm{~m}$ are in the J13 and L9 straights respectively. 


\section{Stripping to Au79+ and Au78+ in J7 Straight}

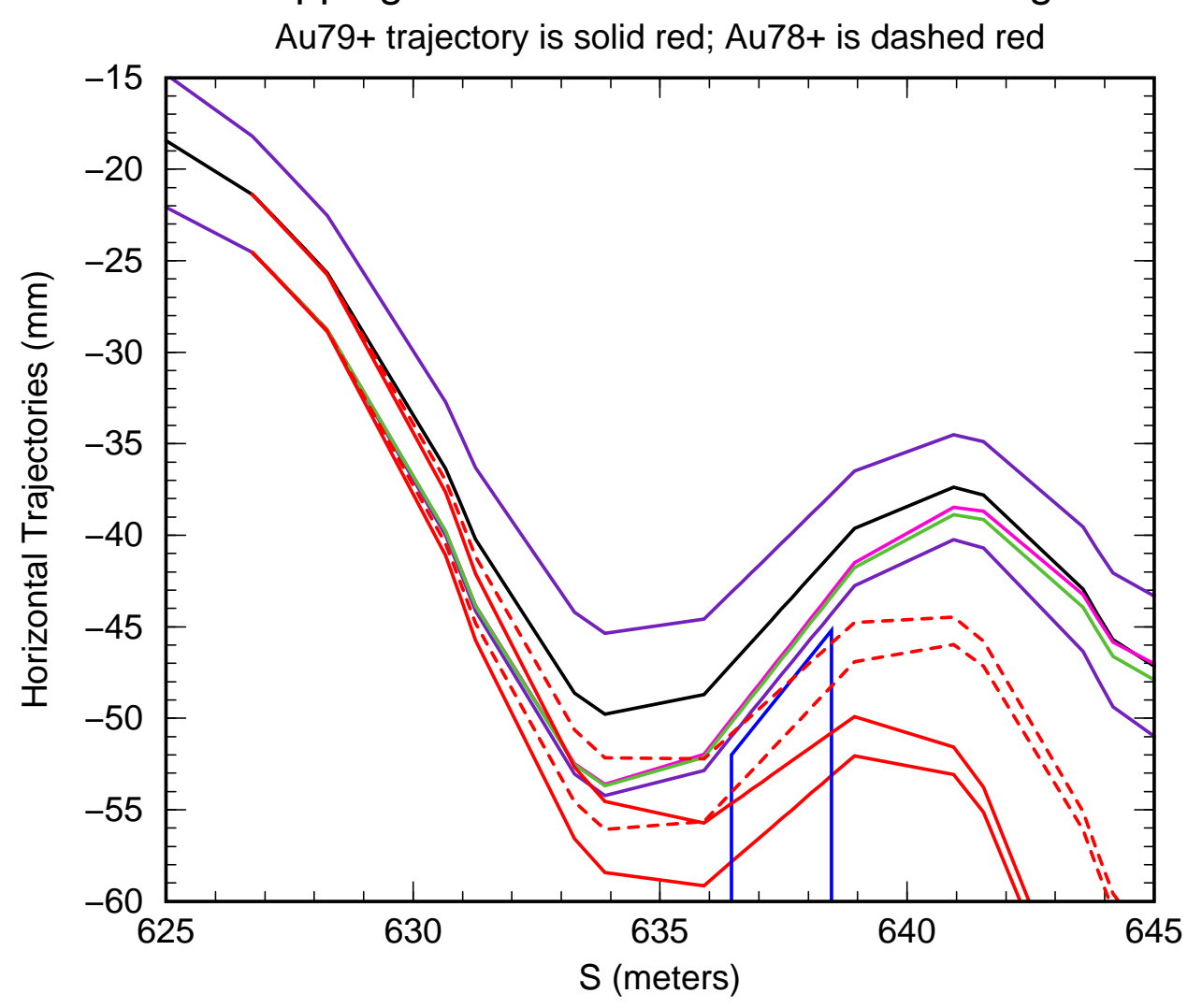

Figure 32: Here we again have stripping from Au77+ to Au79+ and Au78+ in the J7 straight, but now with the dump bump adjusted so that both the Au79+ and the Au78+ ions hit the dump. The solid and dashed red curves are the Au79+ and Au78+ trajectories respectively. Note that there is still clearance between the dump and the circulating beam. The circulating beam emittance is $0.9 \pi \mathrm{mm}$ mrad. 


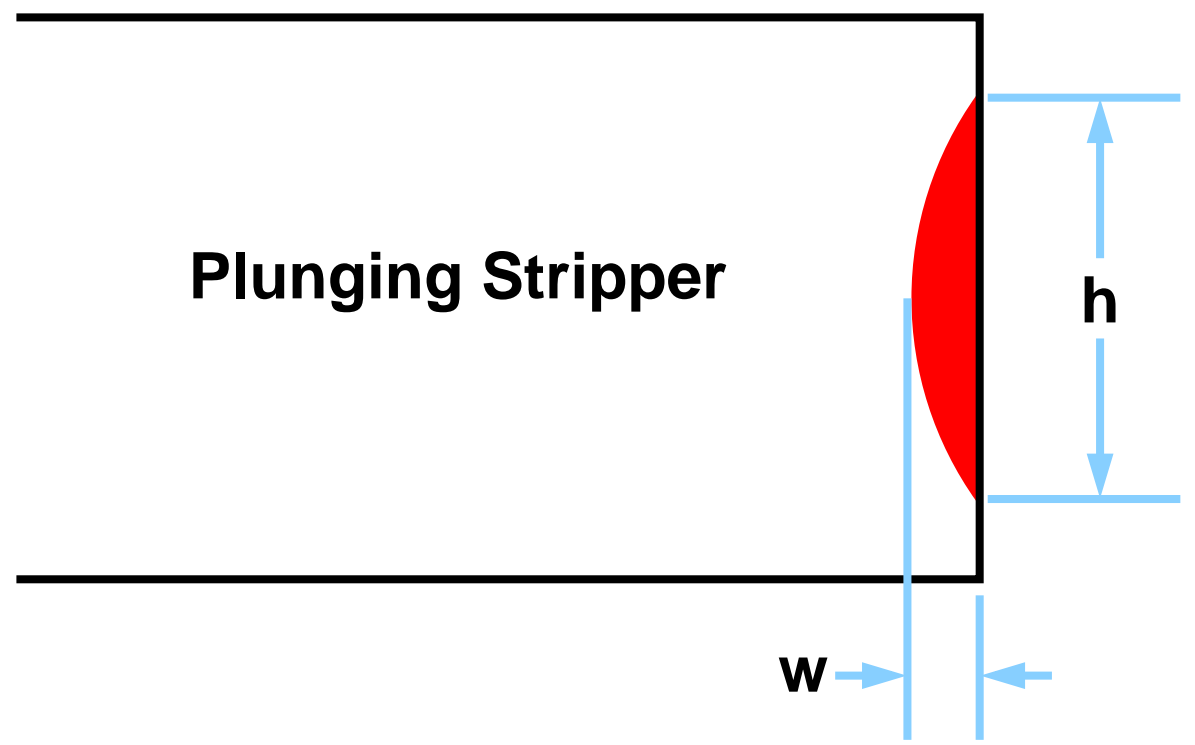

Figure 33: Plunging Stripper. The stripper is plunged into the beam from left to right. The area of ion hits is shown in red. Here $w$ is the maximum distance of hits from the stripper edge and $h$ is the smallest distance along the edge that contains the hits. The area of hits is approximately equal to wh. 


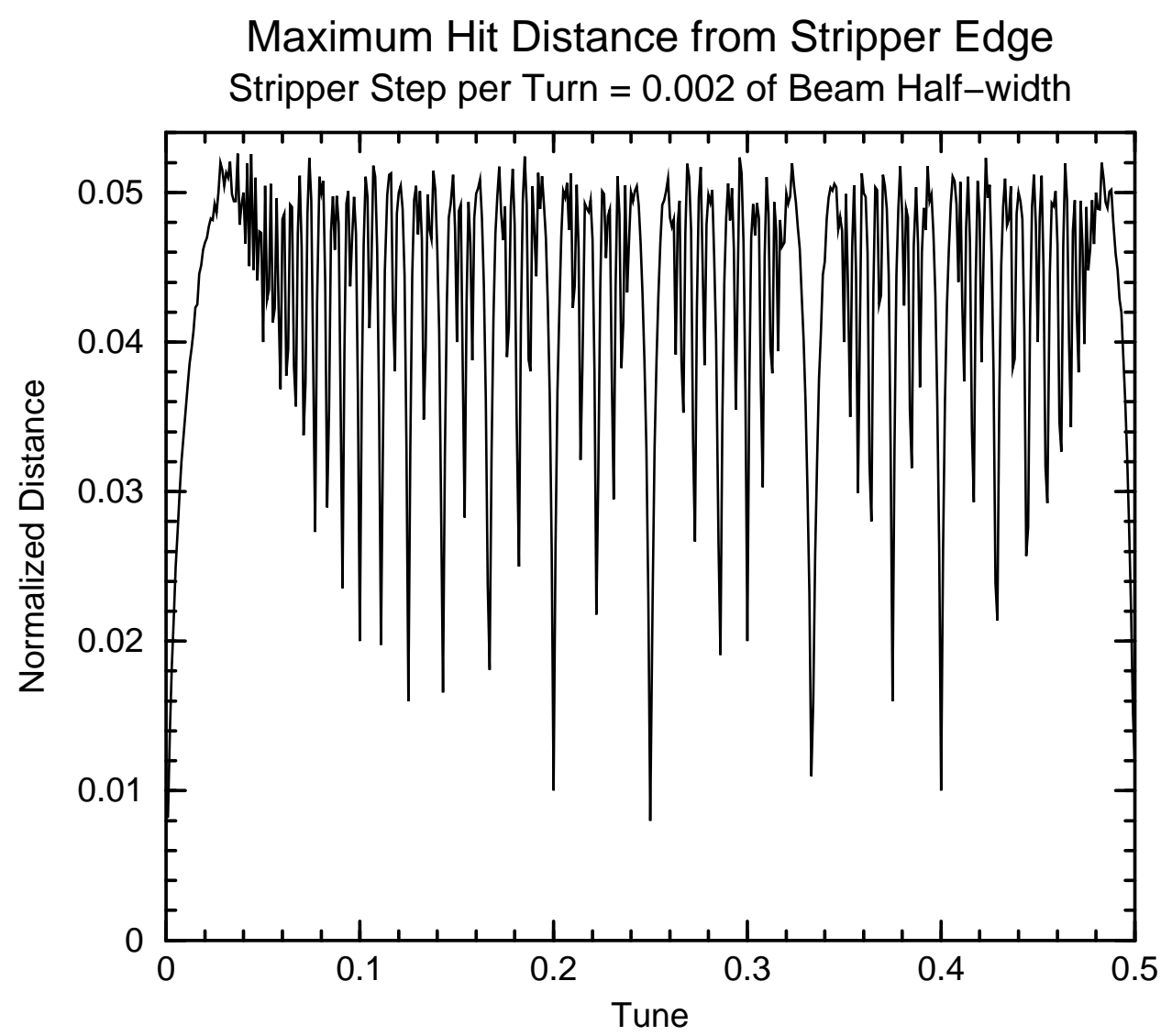

Figure 34: Maximum hit distance from stripper edge versus horizontal tune. This is the result of a simulation in which a stripper is plunged into circulating beam and the maximum distance of ion hits from the stripper edge is recorded as a function of tune. The distance is given in units of beam halfwidth $\sqrt{\epsilon \beta_{0}}$ where $\pi \epsilon$ is the beam emittance and $\beta_{0}$ is the horizontal beta function at the stripper. The beam distribution is assumed to be uniform in $X, X^{\prime}$ space. The stripper is plunged a distance of $\sqrt{\epsilon \beta_{0}}$ into the beam in steps of $0.002 \sqrt{\epsilon \beta_{0}}$ per turn. 


\section{Maximum Hit Distance from Stripper Edge}

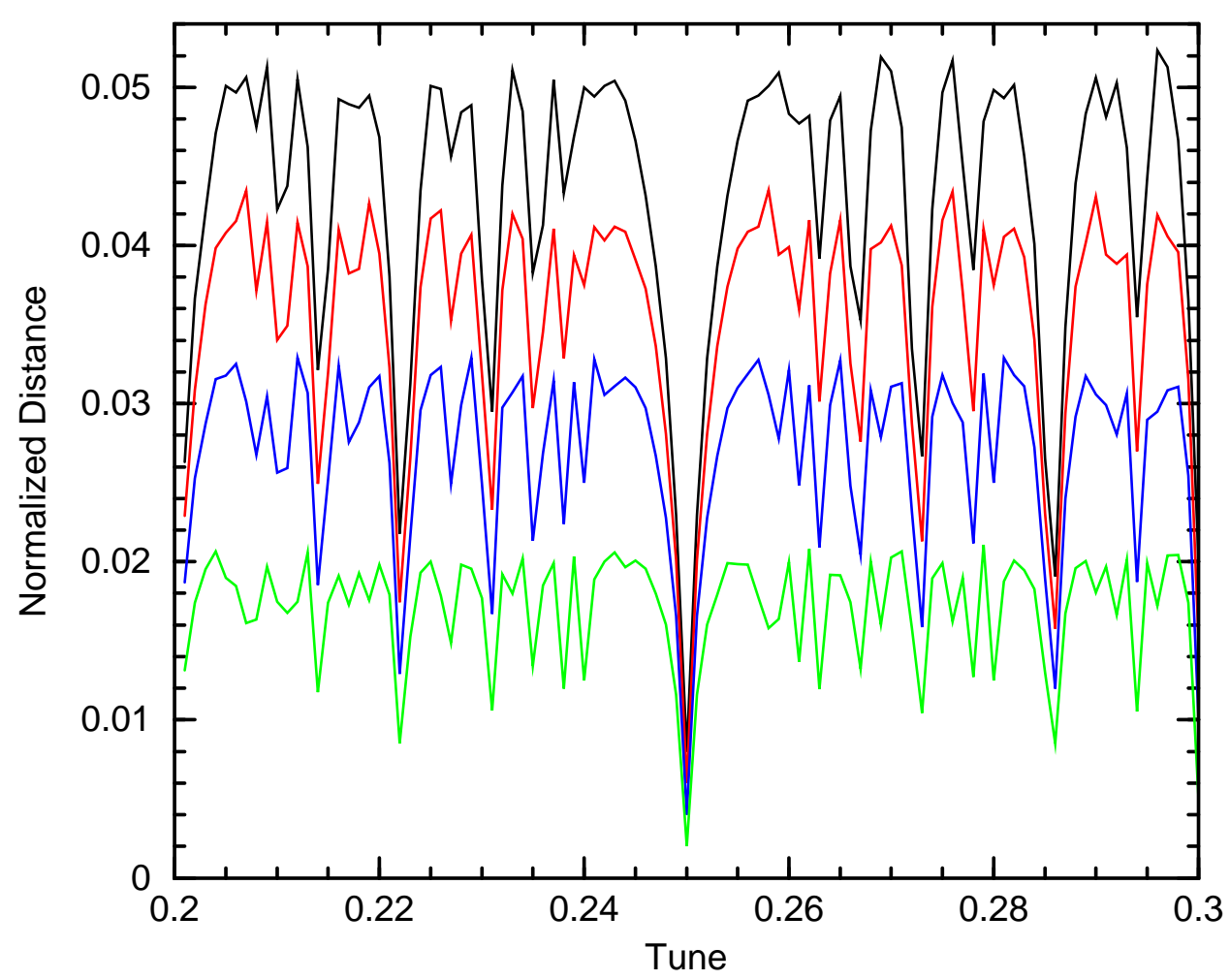

Figure 35: The black curve here is a "zoomed in" view of Figure 34 where the stripper was plunged into the circulating beam in steps of $0.002 \sqrt{\epsilon \beta_{0}}$ per turn. The Red, Blue, and Green curves were obtained with the stripper plunged in steps of $0.0015,0.001$, and $0.0005 \sqrt{\epsilon \beta_{0}}$ per turn. 


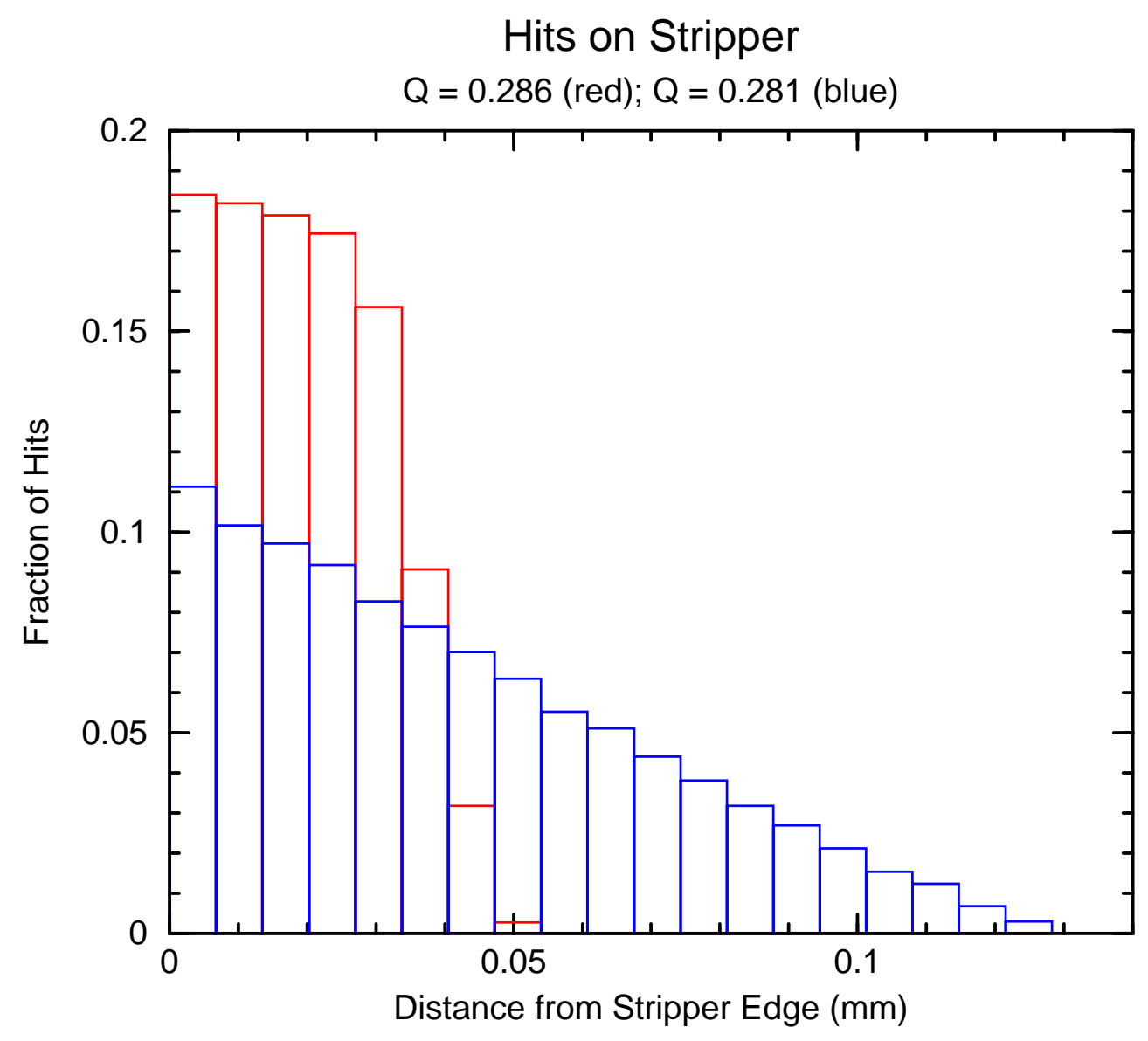

Figure 36: Distribution of hits on the stripper for various tunes. Here the red histogram was obtained with horizontal tune $Q_{H}=0.286$ and the blue with $Q_{H}=0.281$. This is again the result of a simulation in which a stripper is plunged into circulating beam and the distance of ion hits from the stripper edge is recorded. The beam distribution is assumed to be uniform in $X, X^{\prime}$ space. The beam half-width is taken to be $\sqrt{\epsilon \beta_{0}}=2.7 \mathrm{~mm}$ where $\pi \epsilon$ is the beam emittance and $\beta_{0}$ is the horizontal beta function at the stripper. The stripper is plunged a distance of $\sqrt{\epsilon \beta_{0}}$ into the beam in steps of $0.002 \sqrt{\epsilon \beta_{0}}$ per turn. 


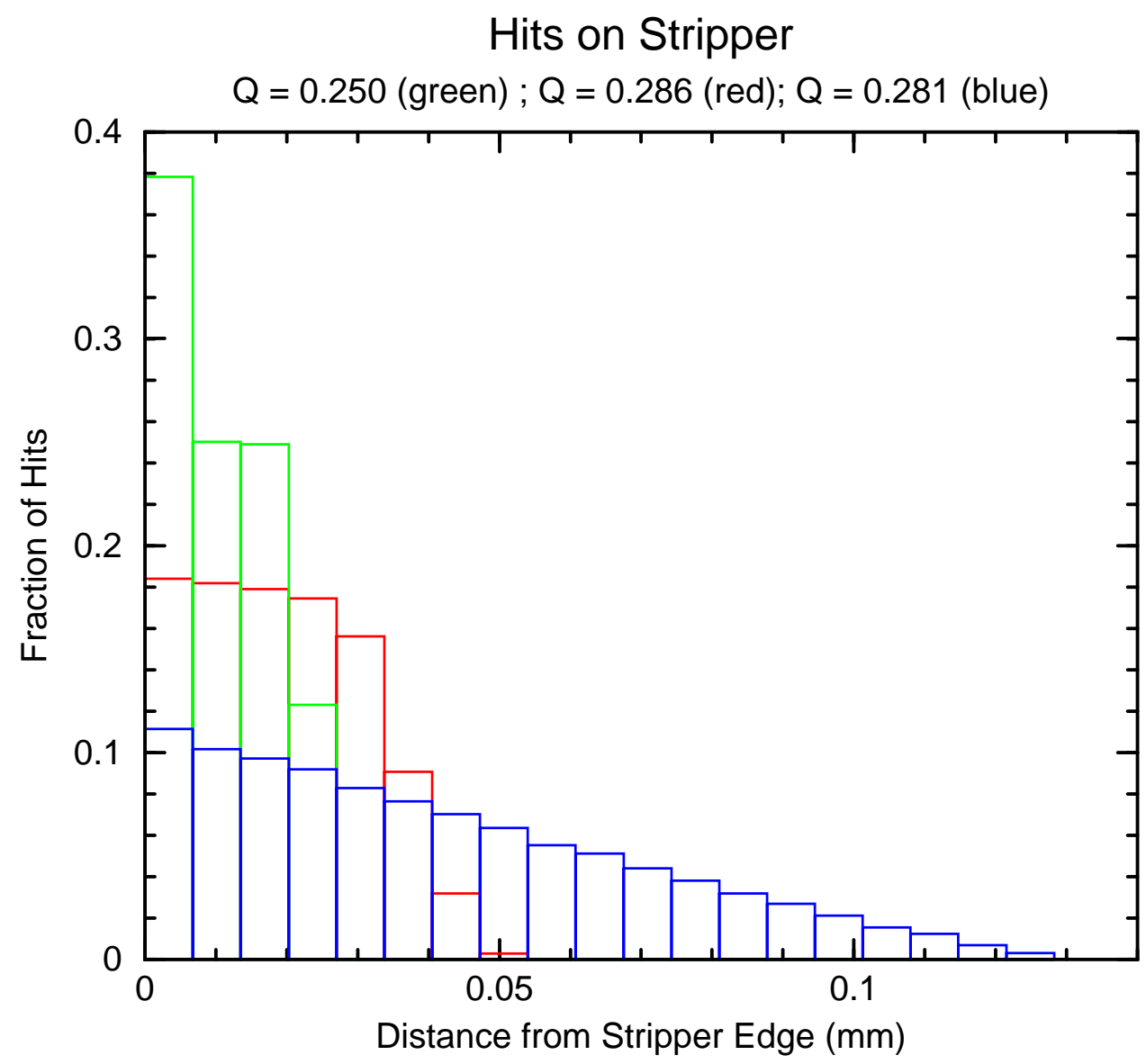

Figure 37: This is just Figure $\mathbf{3 6}$ with the addition of a green histogram for the case in which $Q_{H}=0.250$. 


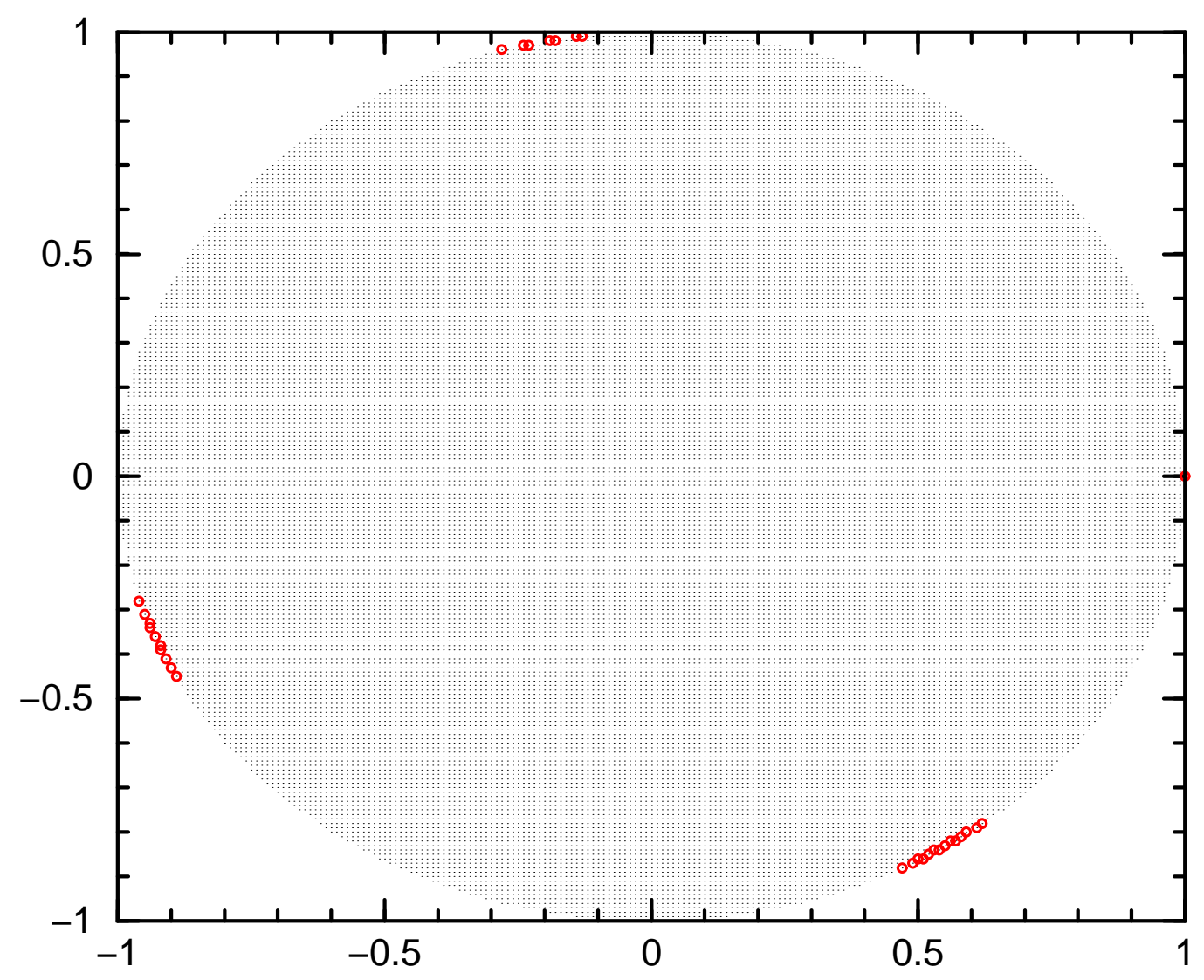

Figure 38: Initial beam distribution in the normalized $X, X^{\prime}$ plane showing all particles (red circles) that have hit the stripper after 3 turns. The stripper step per turn is 0.002 of the beam half-width and 500 steps are required to strip all particles in the distribution. The horizontal tune is $Q_{H}=0.281$. 


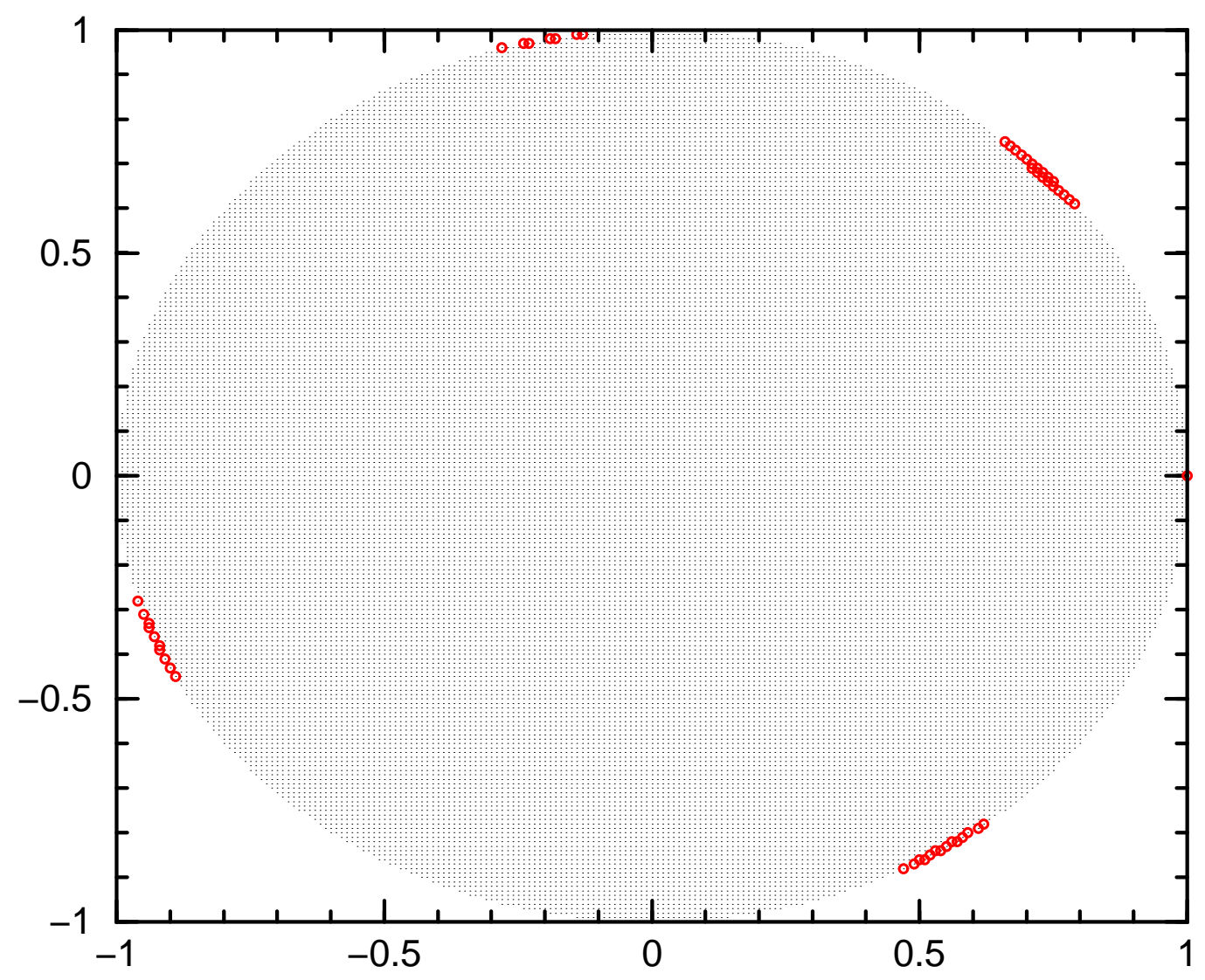

Figure 39: Same as Figure $\mathbf{3 8}$ but showing all particles (red circles) that have hit the stripper after 4 turns. 


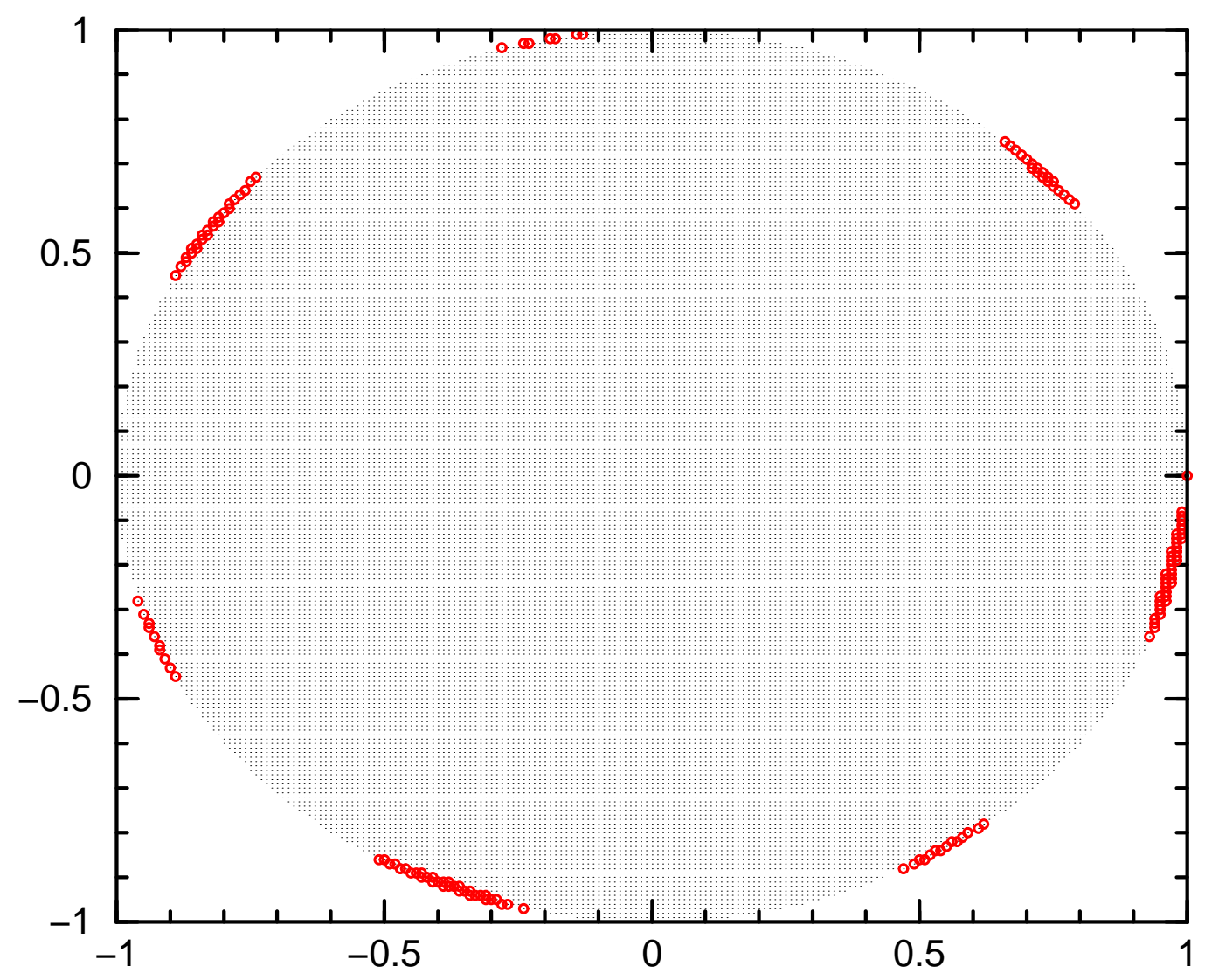

Figure 40: Same as Figure $\mathbf{3 8}$ but showing all particles (red circles) that have hit the stripper after 7 turns. 


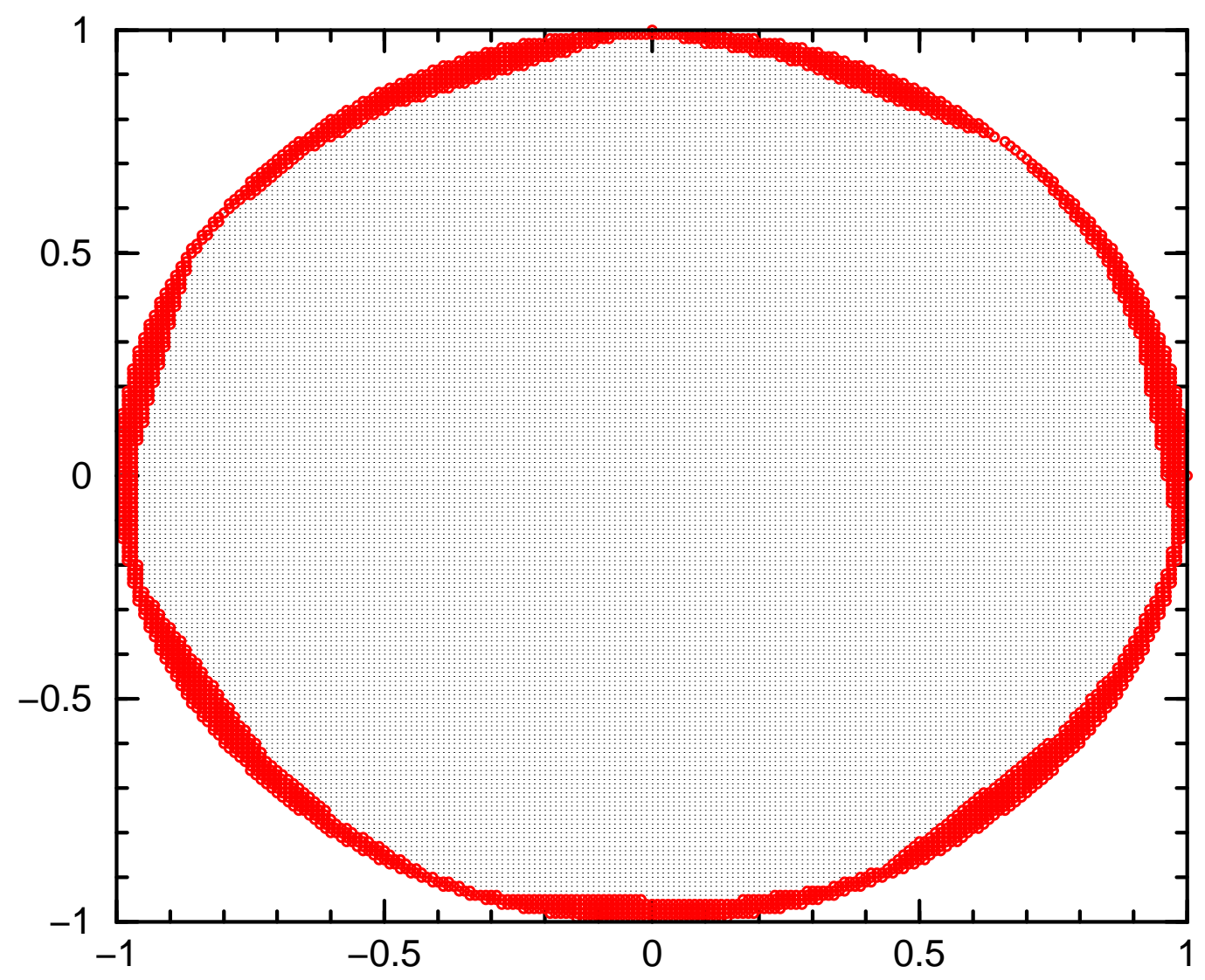

Figure 41: Same as Figure $\mathbf{3 8}$ but showing all particles (red circles) that have hit the stripper after 28 turns. 


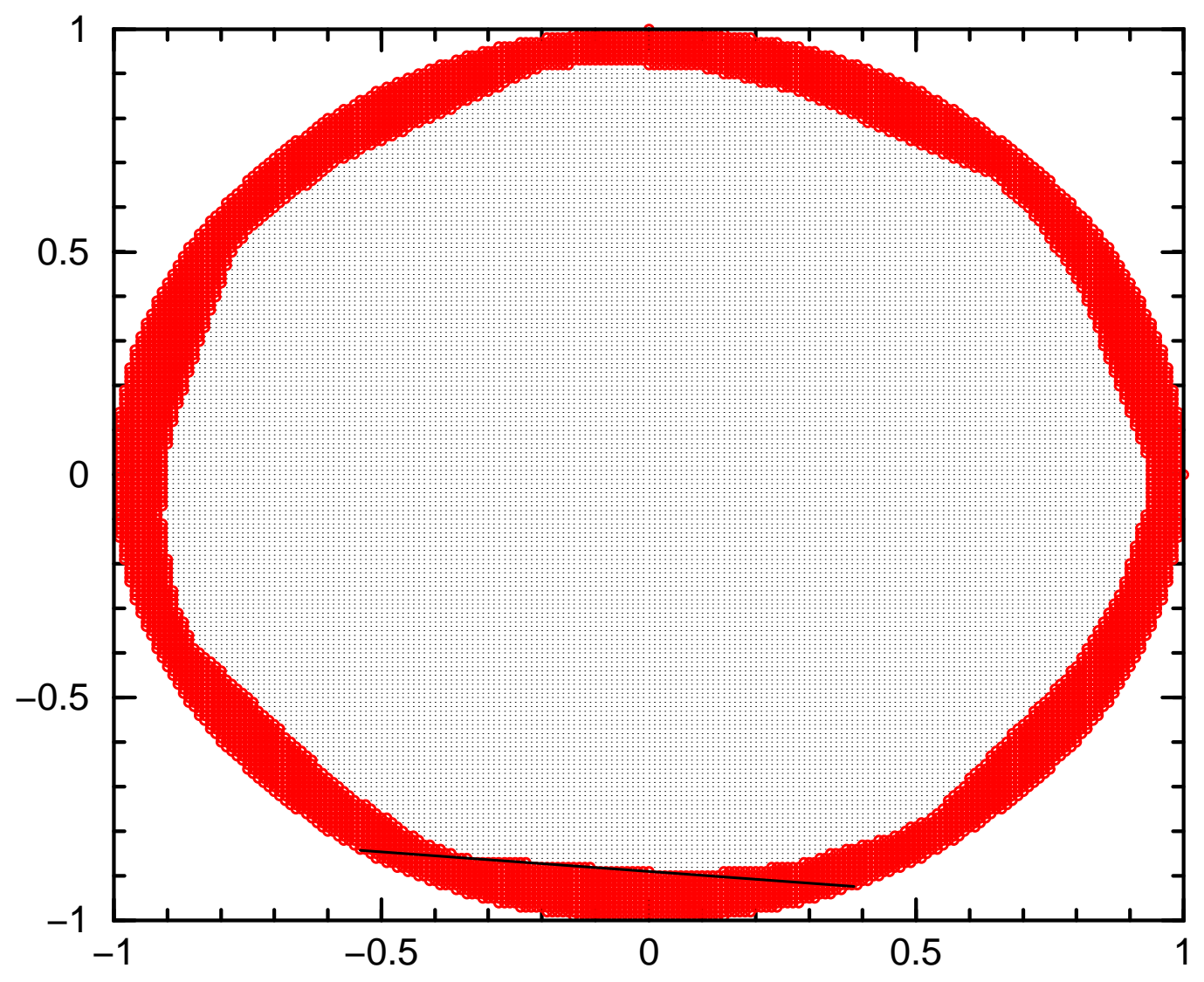

Figure 42: Same as Figure $\mathbf{3 8}$ but showing all particles (red circles) that have hit the stripper after 56 turns. The black line is the rotated image of the stripper edge after 56 turns. 


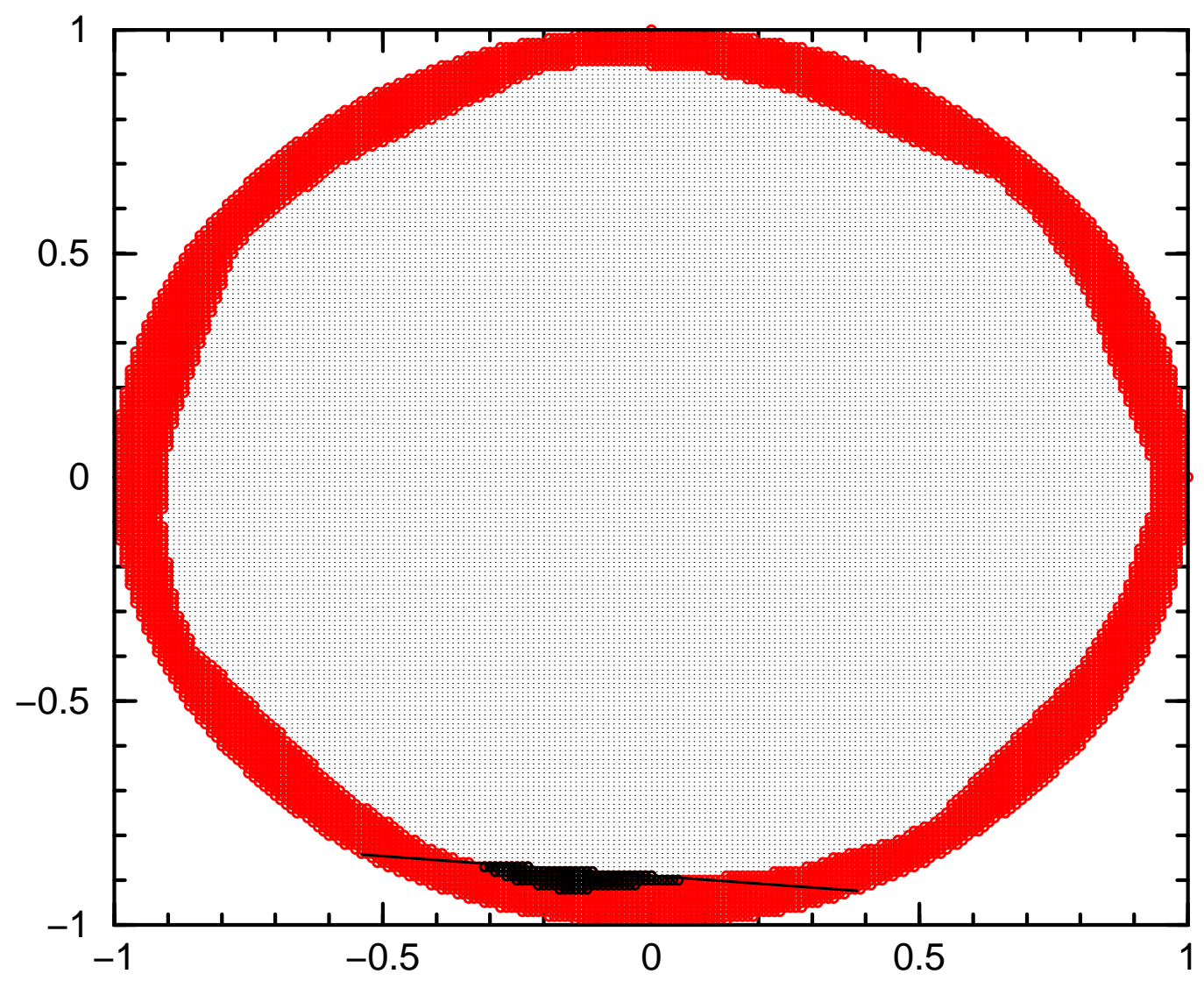

Figure 43: Same as Figure $\mathbf{4 2}$ but with the addition of black circles showing the particles that hit the stripper on the 56th turn. 


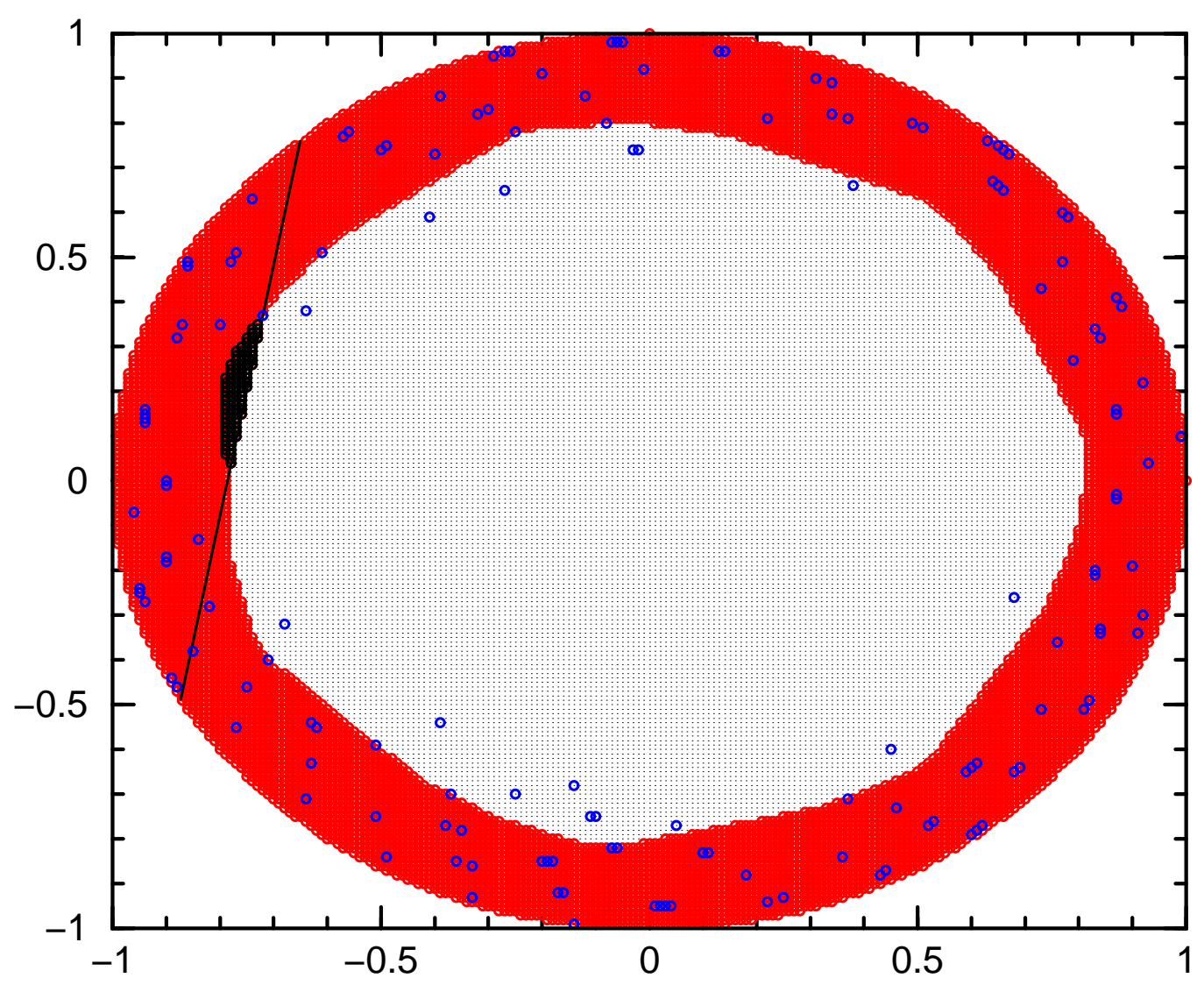

Figure 44: Initial beam distribution showing all particles (red circles) that have hit the stripper after 112 turns. The black line is the rotated image of the stripper edge after 112 turns. The black circles show the particles that hit the stripper on the 112th turn. The blue circles show all particles that hit the stripper at greater than $90 \%$ of the maximum hit distance. 


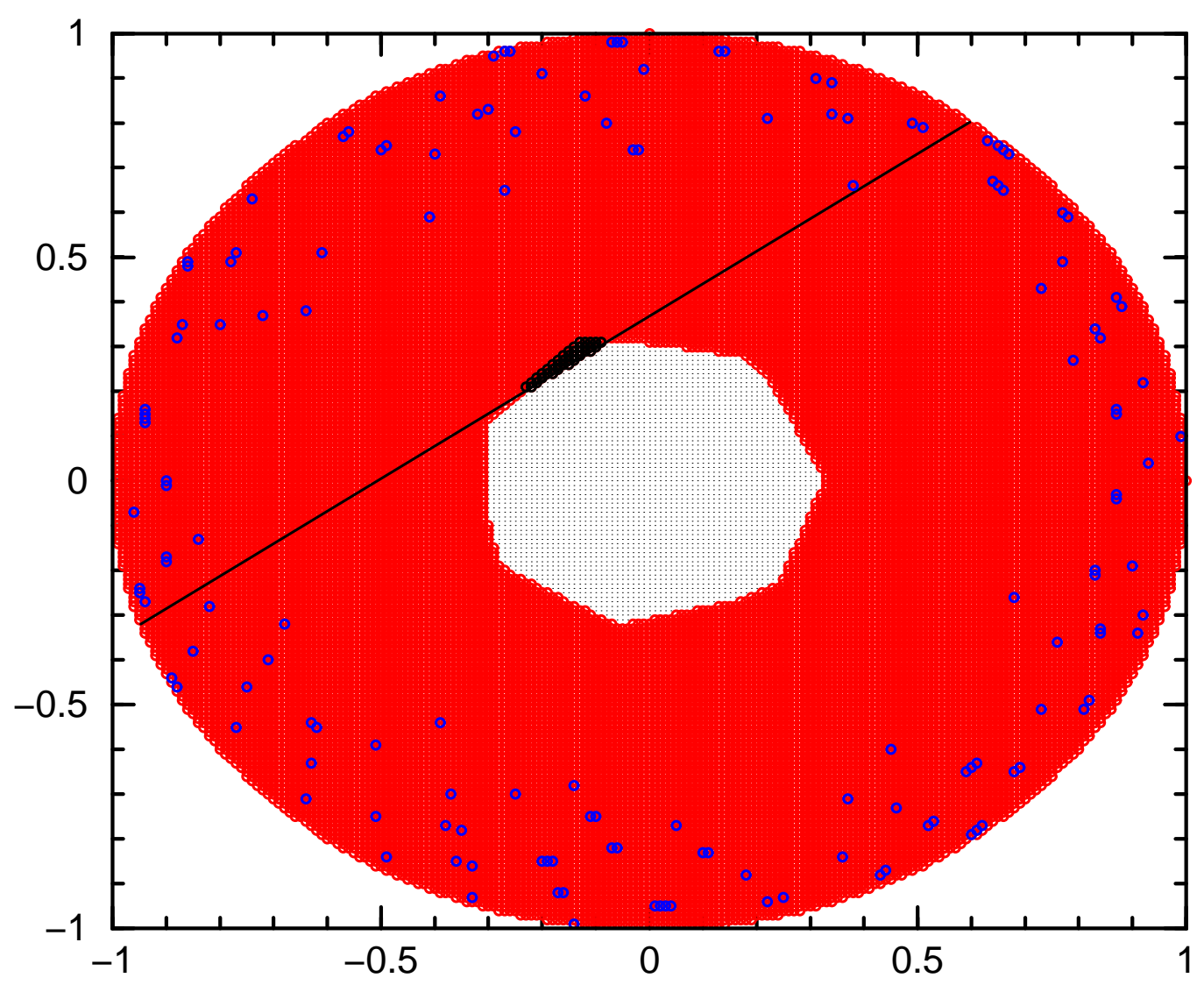

Figure 45: Initial beam distribution showing all particles (red circles) that have hit the stripper after 350 turns. The black line is the rotated image of the stripper edge after 350 turns. The black circles show the particles that hit the stripper on the 350th turn. The blue circles show all particles that hit the stripper at greater than $90 \%$ of the maximum hit distance. 


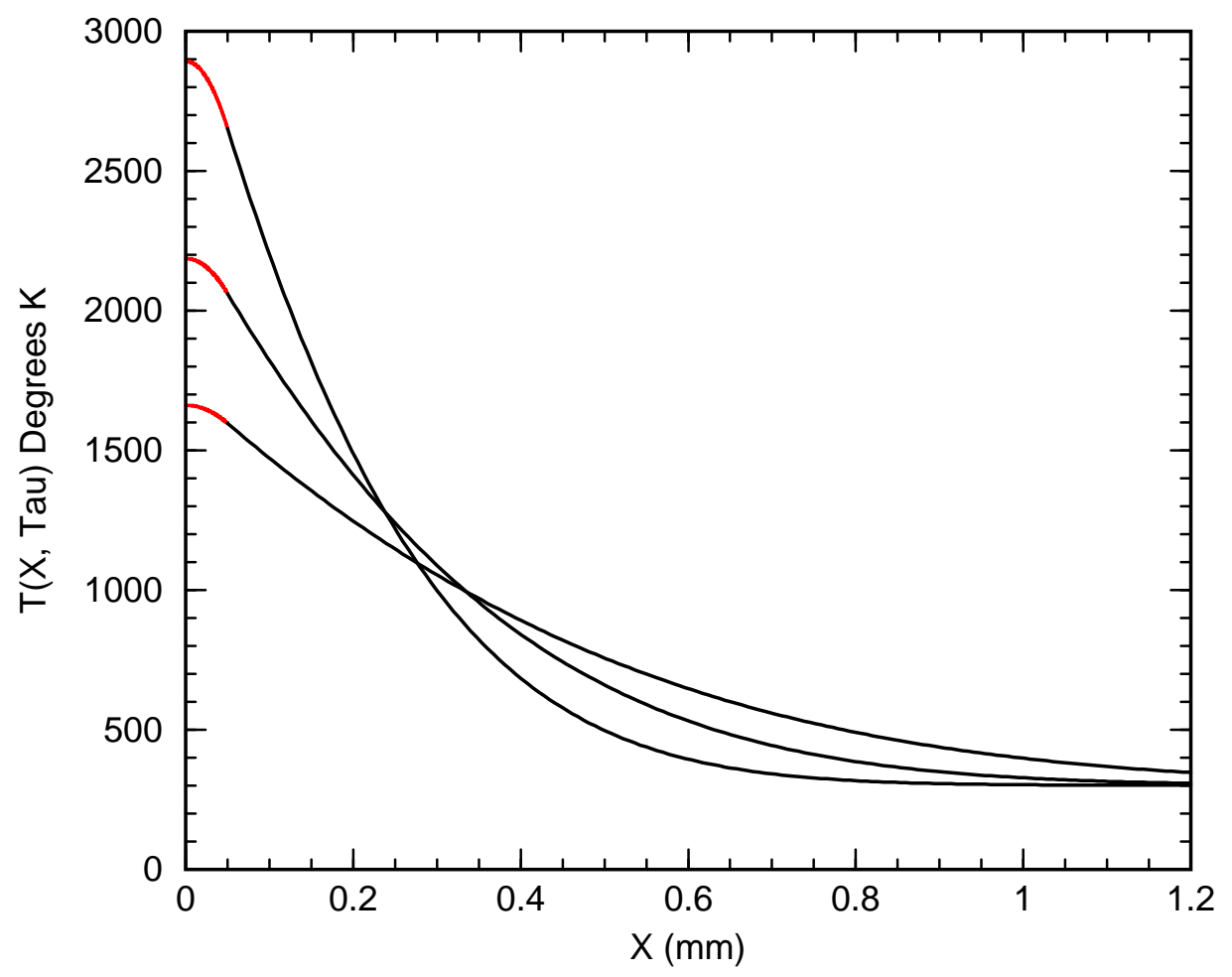

Figure 46: Foil temperature $T(x, \tau)$ obtained from (107) and (108) with $w=0.05 \mathrm{~mm}, h=10 \mathrm{~mm}$ and initial temperature $T_{0}=300 \mathrm{~K}$. The energy deposition time $\tau$ is $1.35,2.7$, and 5.4 ms respectively for the upper, middle, and lower curves. The red portion of each curve extends from $x=0$ to $x=w$. 


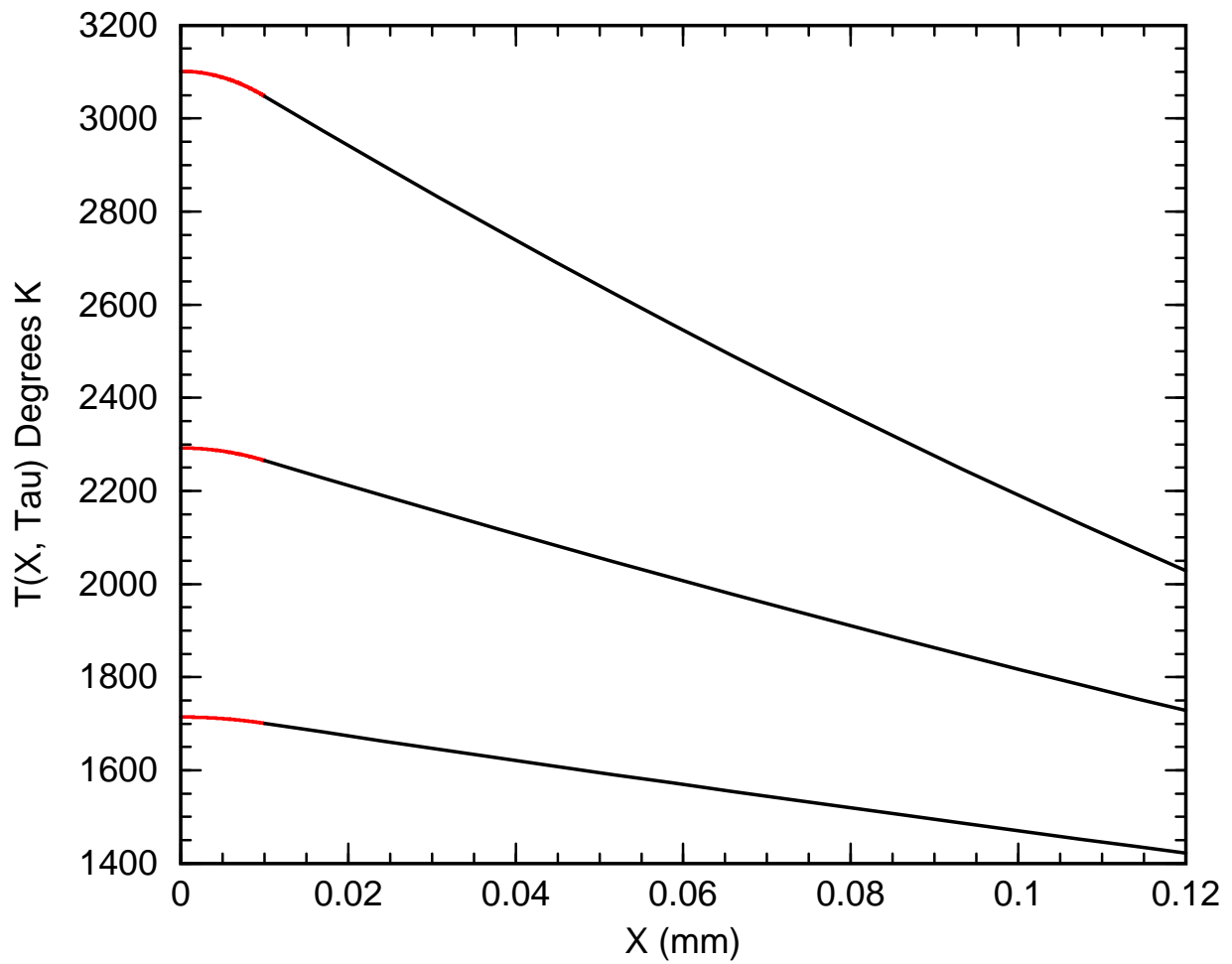

Figure 47: Foil temperature $T(x, \tau)$ obtained from (107) and (108) with $w=0.01 \mathrm{~mm}, h=10 \mathrm{~mm}$ and initial temperature $T_{0}=300 \mathrm{~K}$. The energy deposition time $\tau$ is $1.35,2.7$, and $5.4 \mathrm{~ms}$ respectively for the upper, middle, and lower curves. The red portion of each curve extends from $x=0$ to $x=w$. 


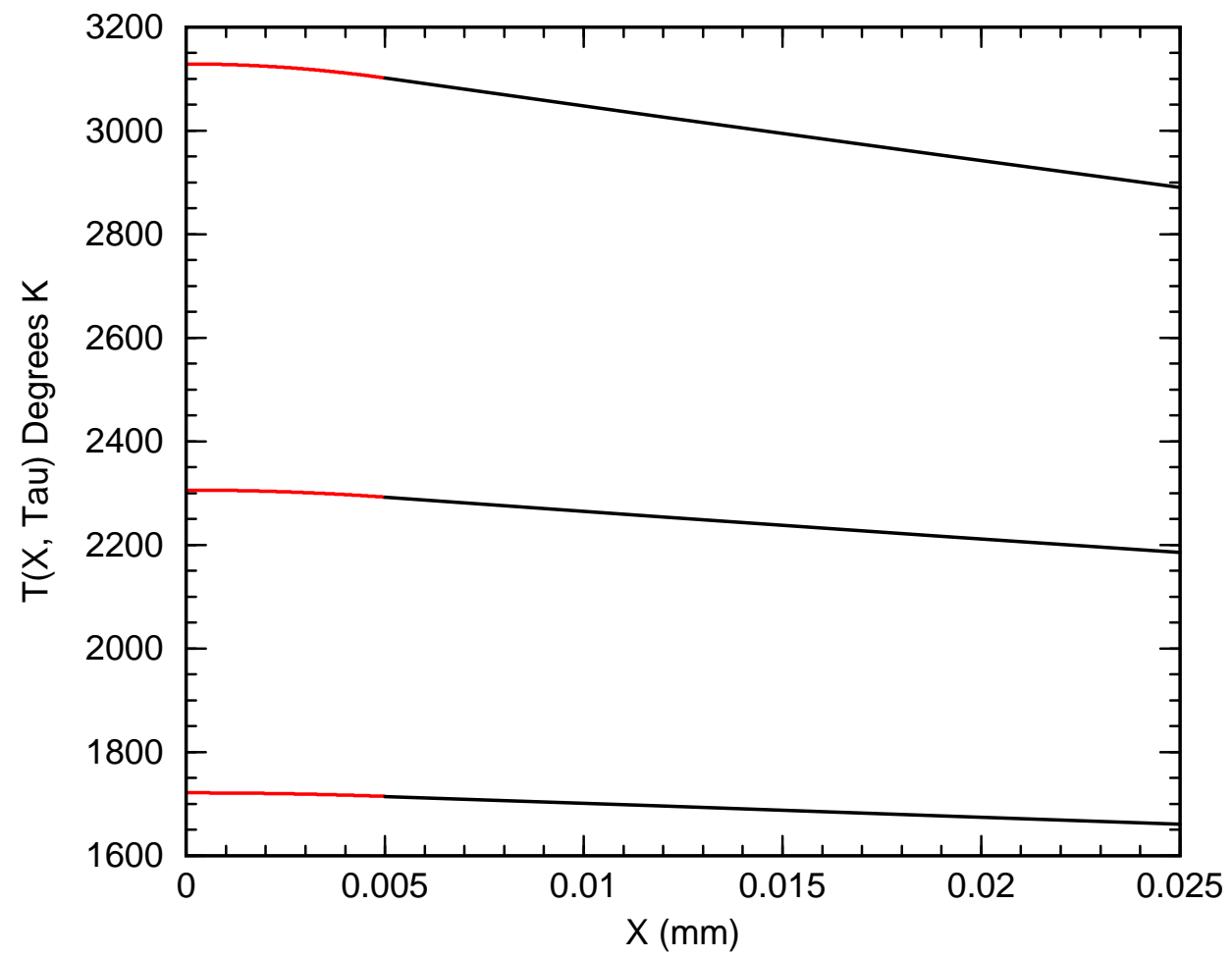

Figure 48: Foil temperature $T(x, \tau)$ obtained from (107) and (108) with $w=0.005 \mathrm{~mm}, h=10 \mathrm{~mm}$ and initial temperature $T_{0}=300 \mathrm{~K}$. The energy deposition time $\tau$ is $1.35,2.7$, and $5.4 \mathrm{~ms}$ respectively for the upper, middle, and lower curves. The red portion of each curve extends from $x=0$ to $x=w$. 


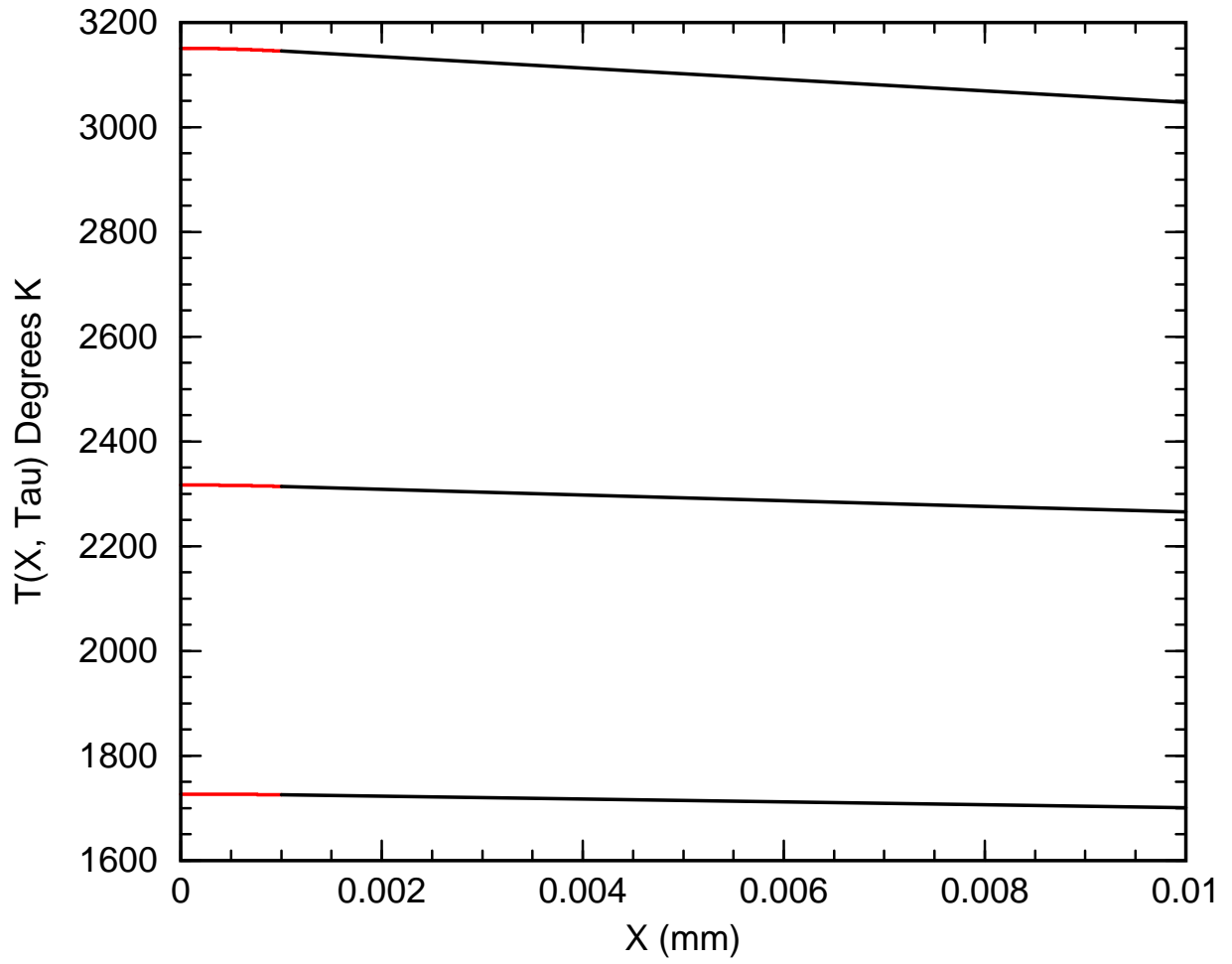

Figure 49: Foil temperature $T(x, \tau)$ obtained from (107) and (108) with $w=0.001 \mathrm{~mm}, h=10 \mathrm{~mm}$ and initial temperature $T_{0}=300 \mathrm{~K}$. The energy deposition time $\tau$ is $1.35,2.7$, and $5.4 \mathrm{~ms}$ respectively for the upper, middle, and lower curves. The red portion of each curve extends from $x=0$ to $x=w$. 


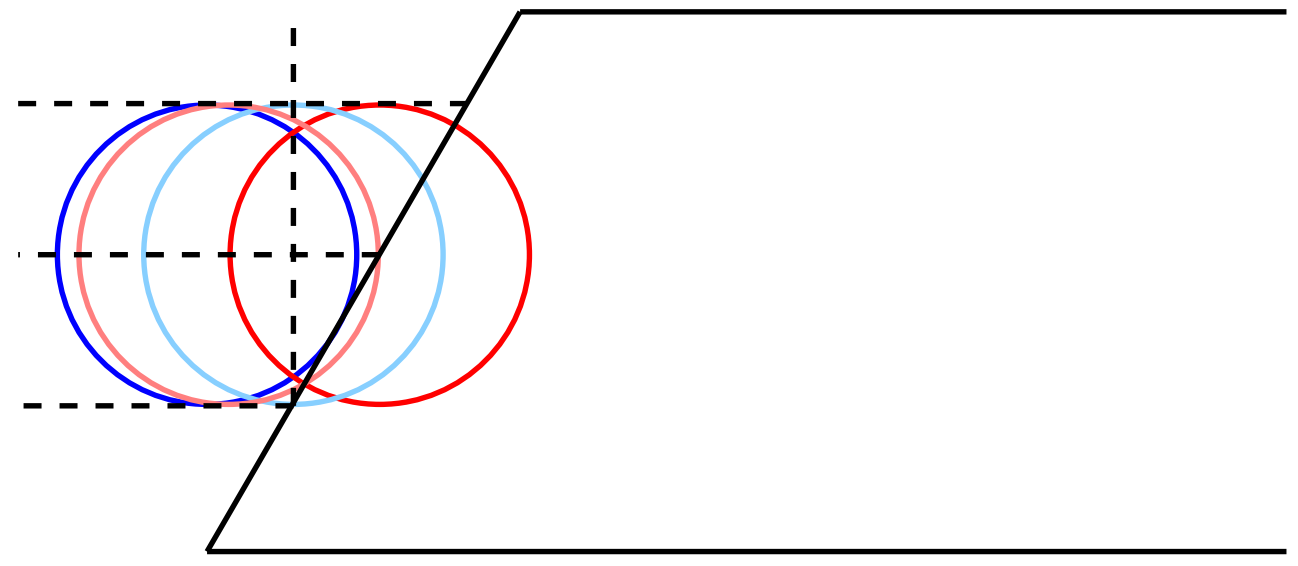

Figure 50: Plunging Stripper Edge at $\theta=60$ degrees. Width of projection of circle of radius $R$ on edge is $W=2 R / \sin \theta$. Because of vertical motion, particles hit the lower half of the inclined edge first. As a result, the distance along the edge that is hit by beam is just $W / 2$.

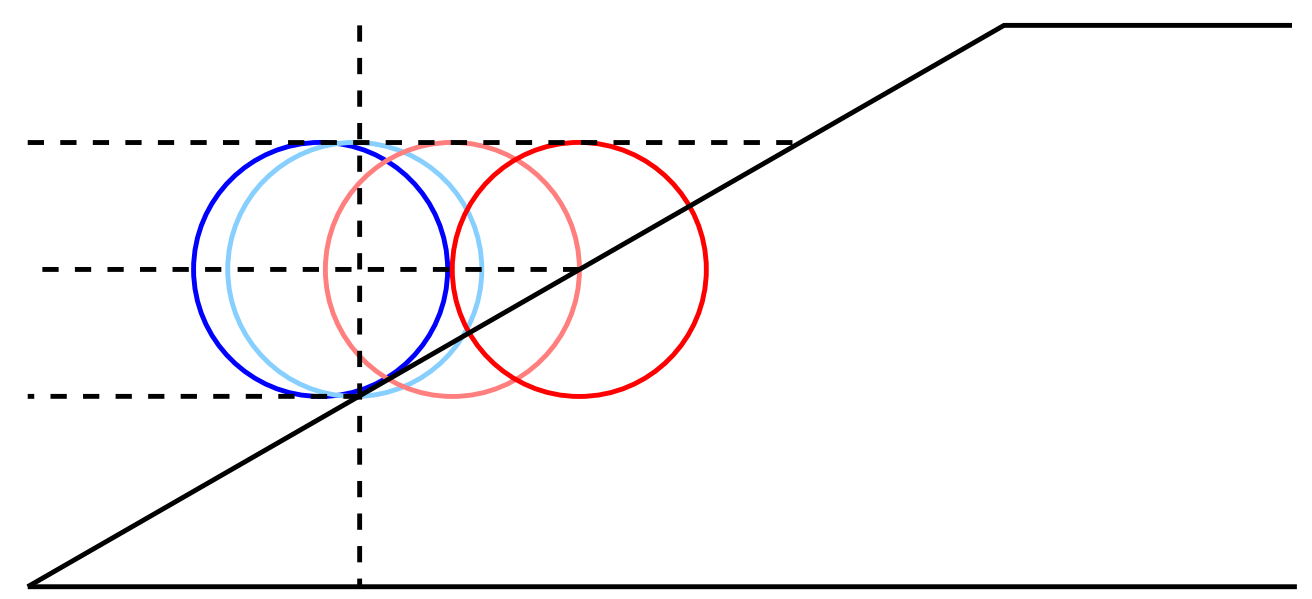

Figure 51: Plunging Stripper Edge at 30 degrees. 


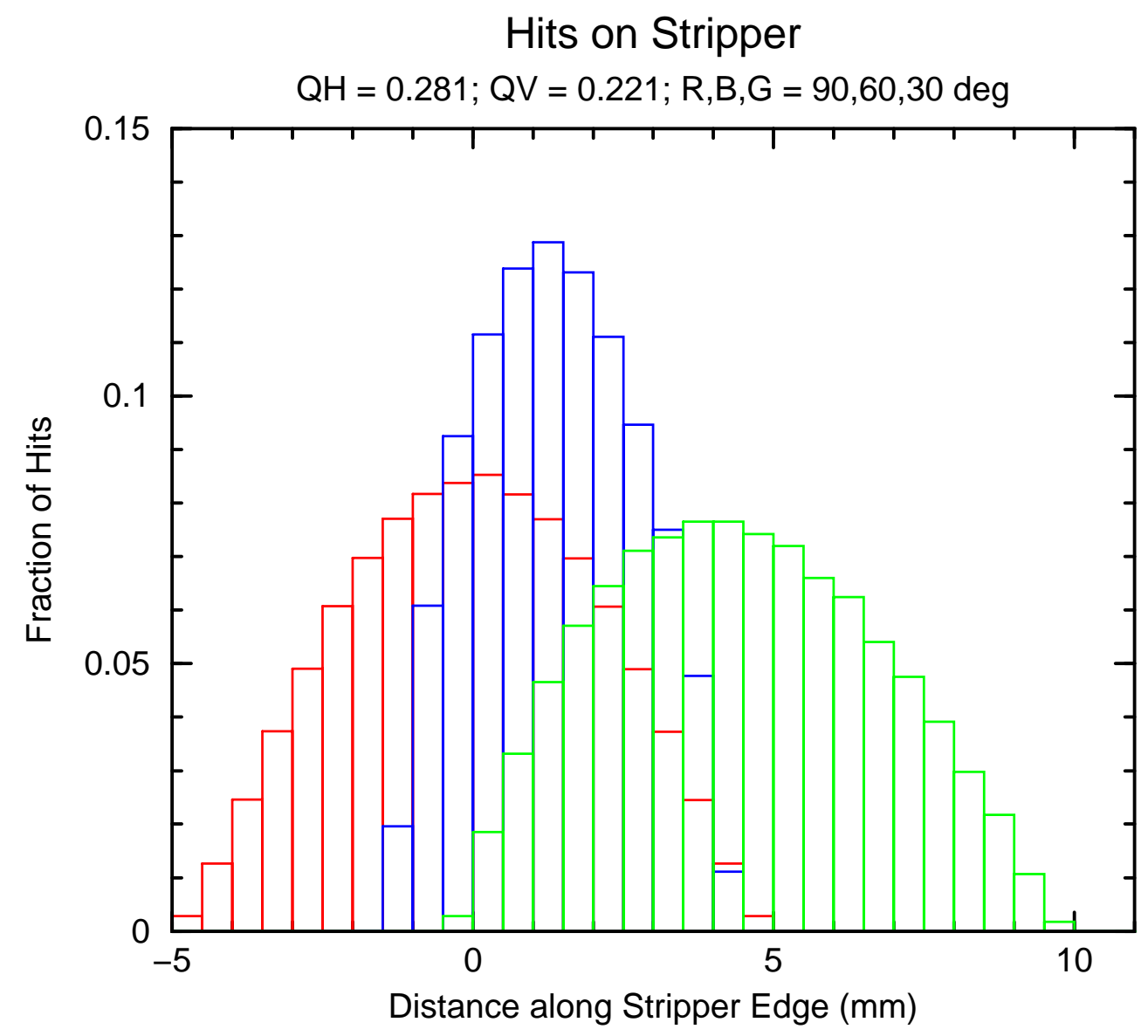

Figure 52: Distribution of hits on stripper. Red, Blue and Green histograms were obtained from simulations with stripper edge at $\theta=90,60$, and 30 degrees respectively. $\theta$ is the angle the edge makes with respect to the direction of stripper motion. Initial distribution was largest ball (in normalized coordinates) contained in a four-dimensional cube uniformly populated with $40^{4}$ particles. We assumed $\sqrt{\epsilon \beta}=2.7$ and $5.0 \mathrm{~mm}$ in the horizontal and vertical planes respectively. The stripper was moved into the circulating beam in horizontal steps of $0.002 \sqrt{\epsilon \beta}$ per turn $(2.7 \mu \mathrm{s})$ which gives a speed of 2.0 $\mathrm{mm} / \mathrm{ms}$. The times over which hits occur on the stripper are then 1.35, 1.98, and $4.54 \mathrm{~ms}$ for $\theta=90,60$, and 30 degrees respectively. During these times the stripper moves $2.70,3.95$ and $9.07 \mathrm{~mm}$ respectively in the horizontal direction. The horizontal and vertical tunes were taken to be $Q_{H}=0.281$ and $Q_{V}=0.221$. 


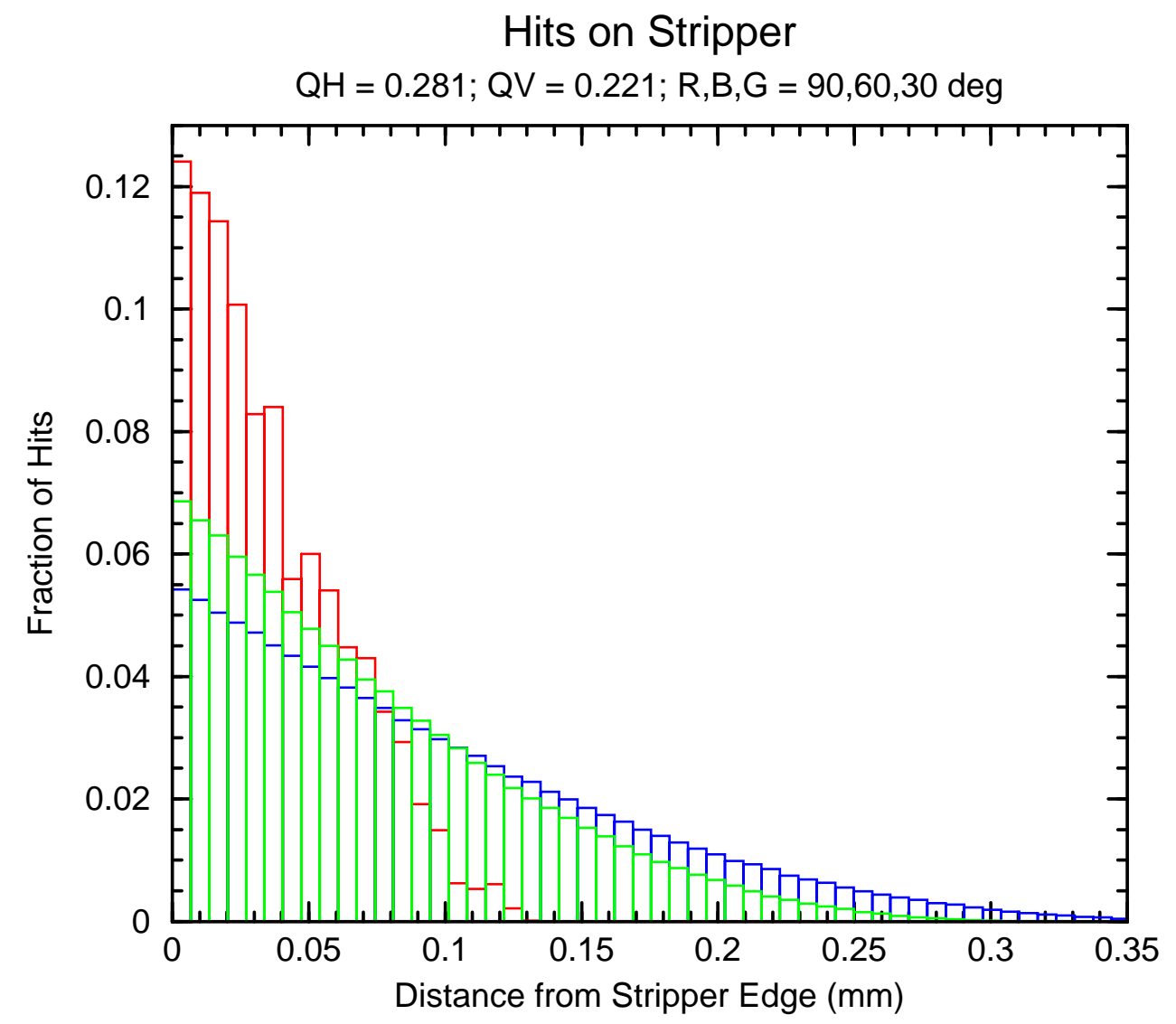

Figure 53: Same simulation as in Figure 52, but showing the fraction of hits as a function of distance from the stripper edge. The Red, Blue and Green histograms were obtained with $\theta=90,60$, and 30 degrees respectively. 


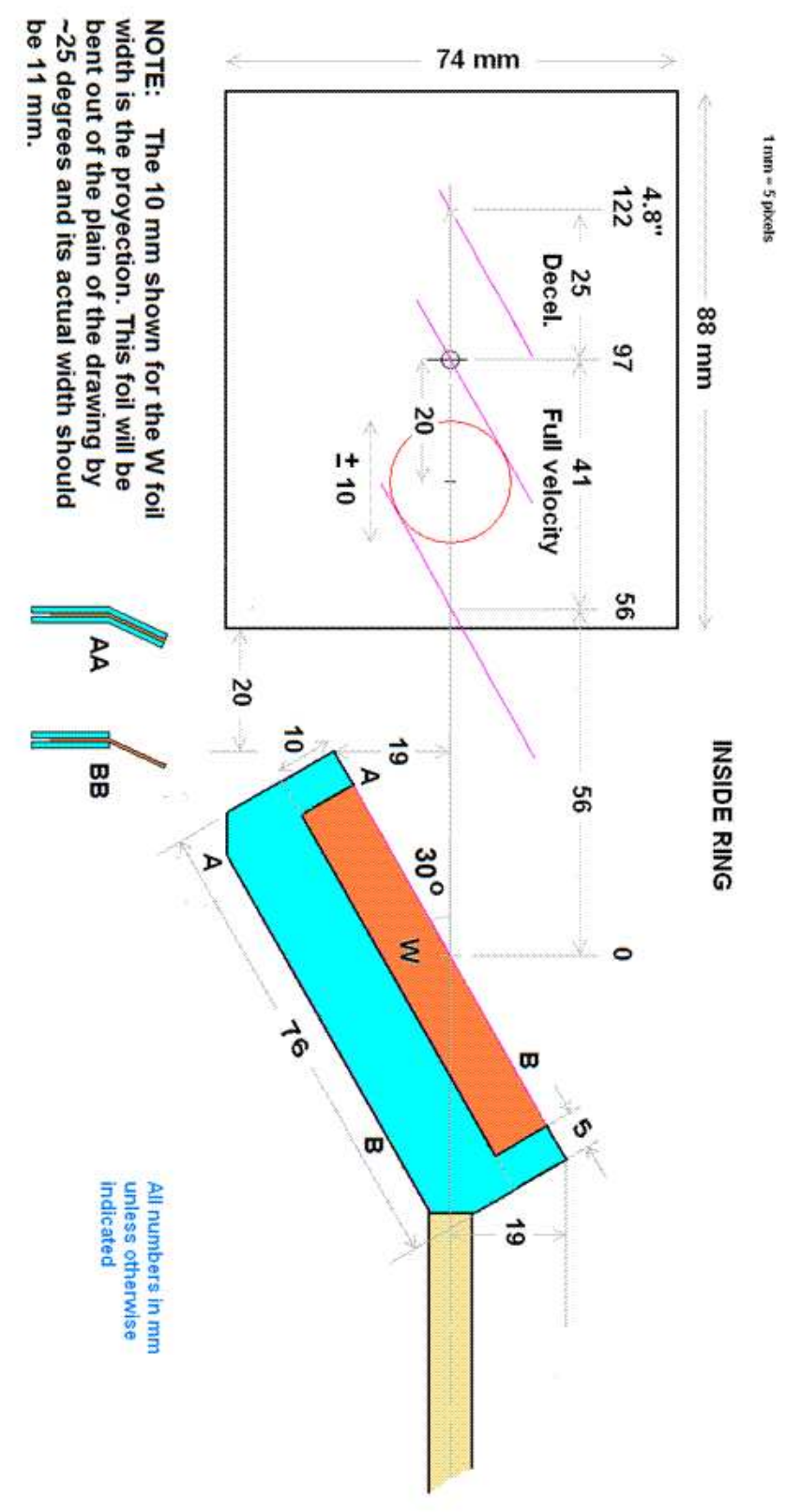

Figure 54: Peter Thieberger's plunging stripper design with the tungsten stripping foil plunged from the inside side of the ring. 


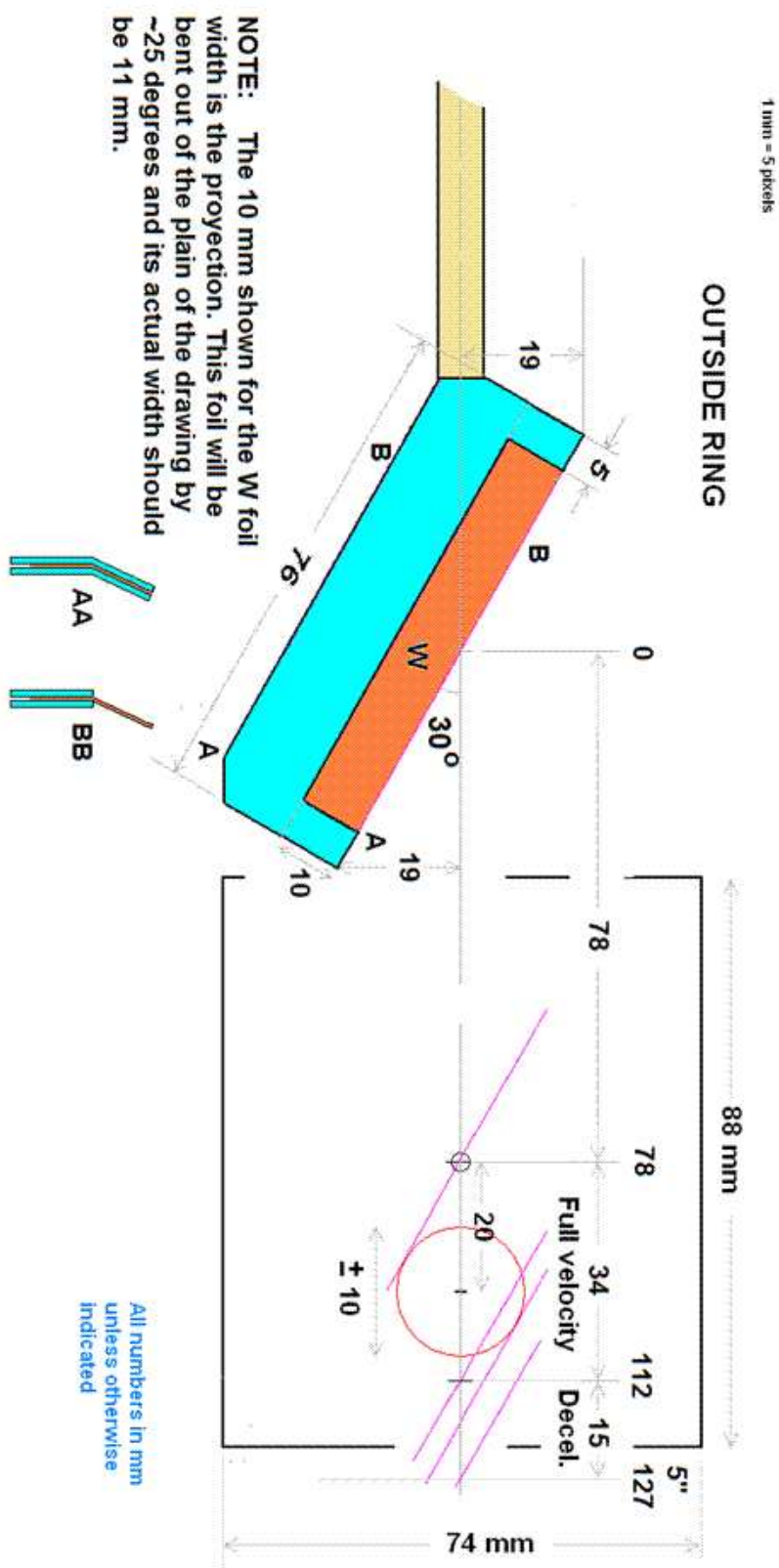

Figure 55: Peter Thieberger's plunging stripper design with the tungsten stripping foil plunged from the outside. 


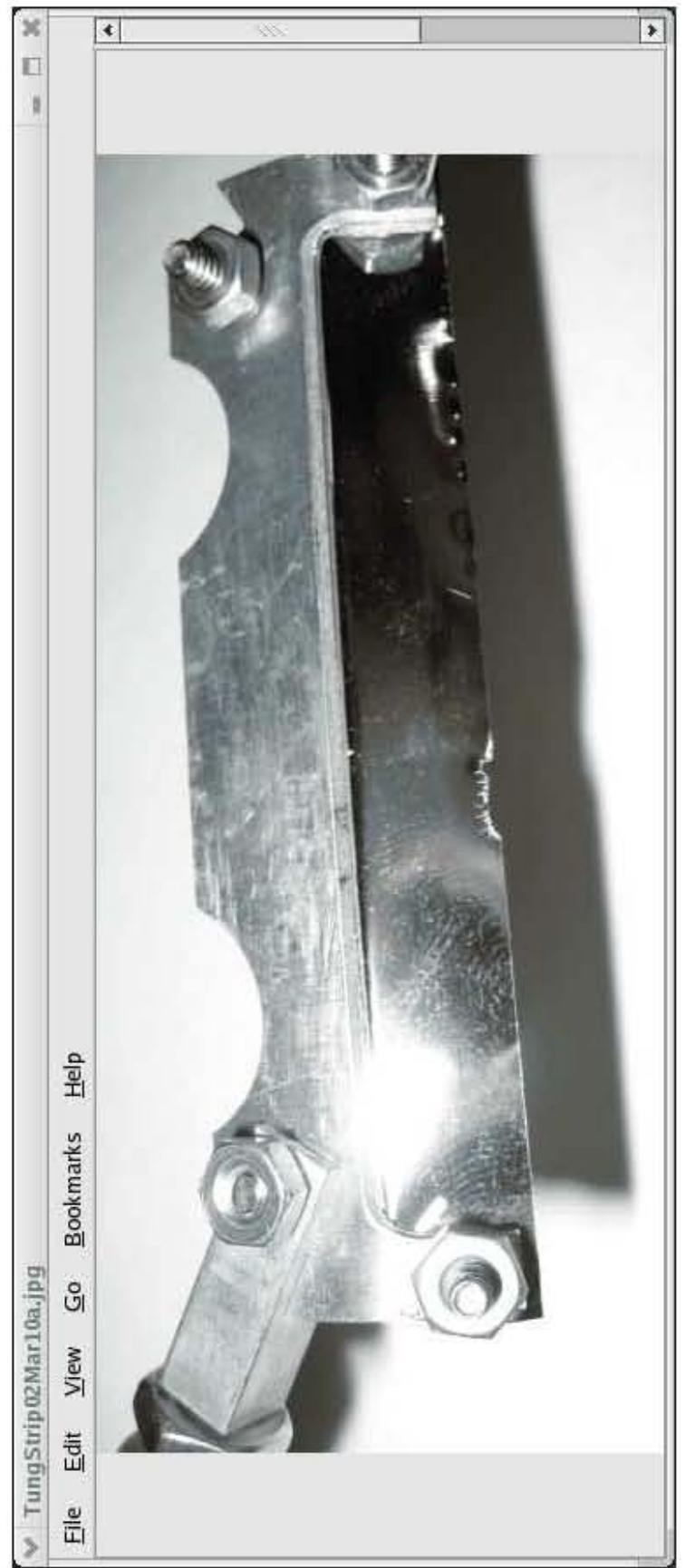

Figure 56: Inside plunging stripper after several weeks of running. 


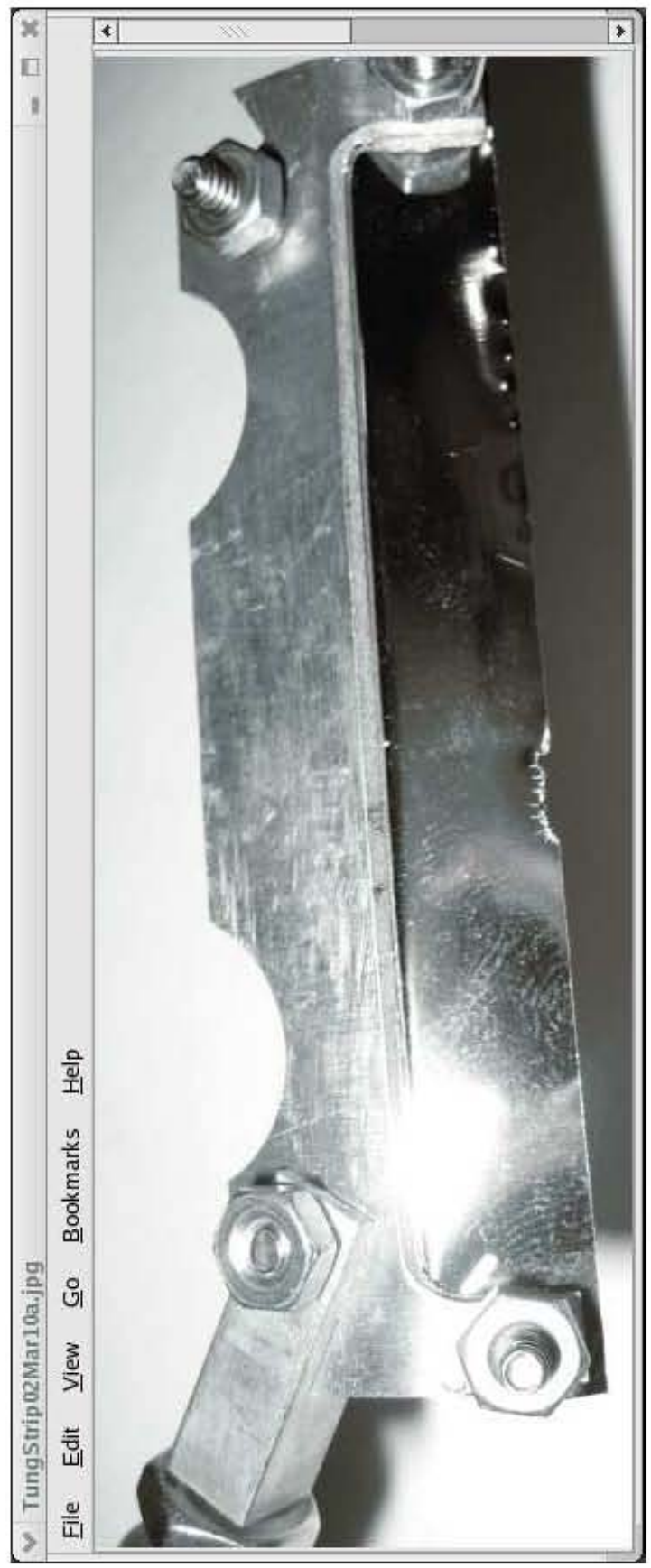

Figure 57: Slightly magnified view of Figure 56. 


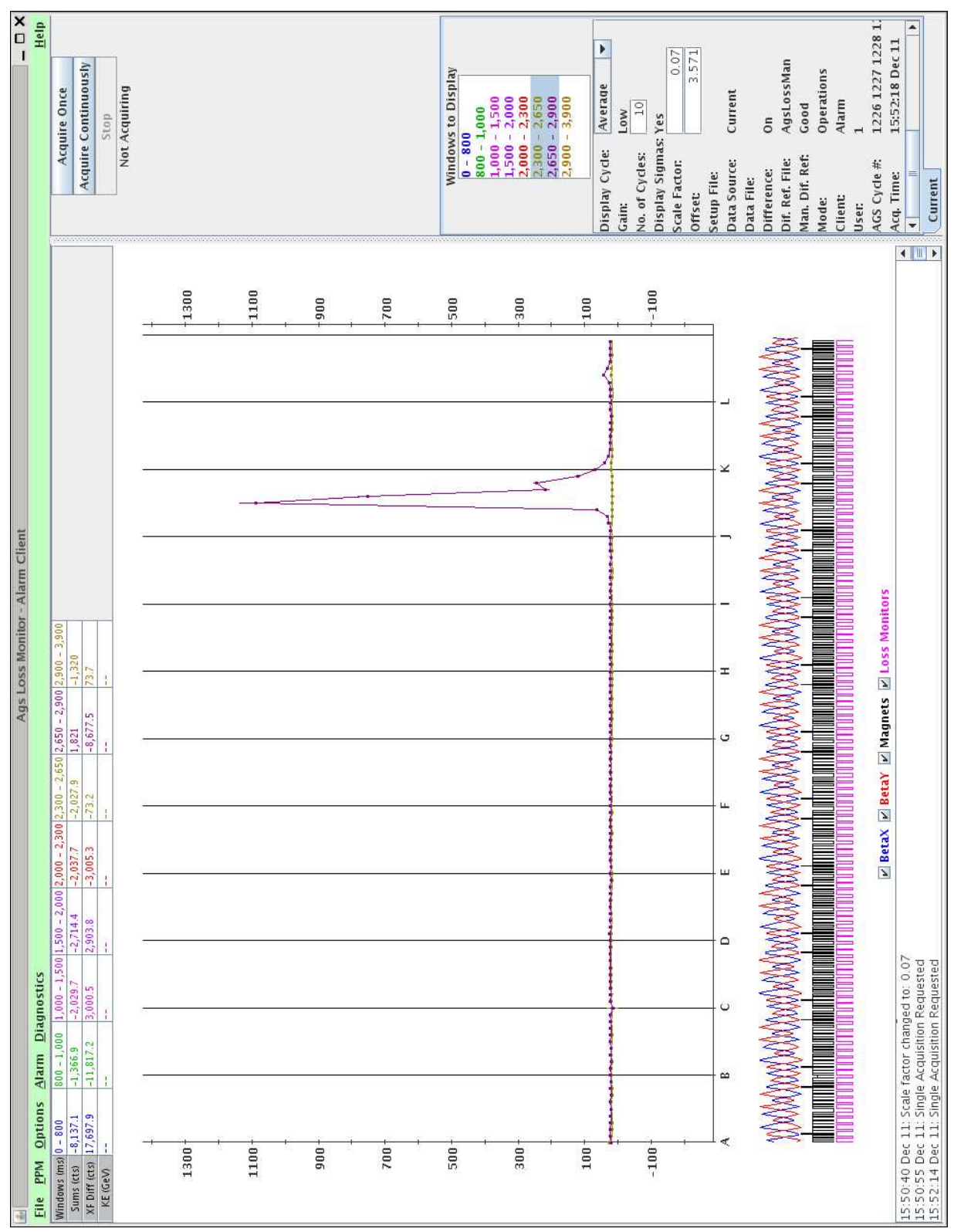

Figure 58: Loss pattern in AGS with some $3.5 \times 10^{9}$ gold ions being put into the J10 dump at extraction energy. Here the J7 stripper is not being plunged into the circulating beam. 


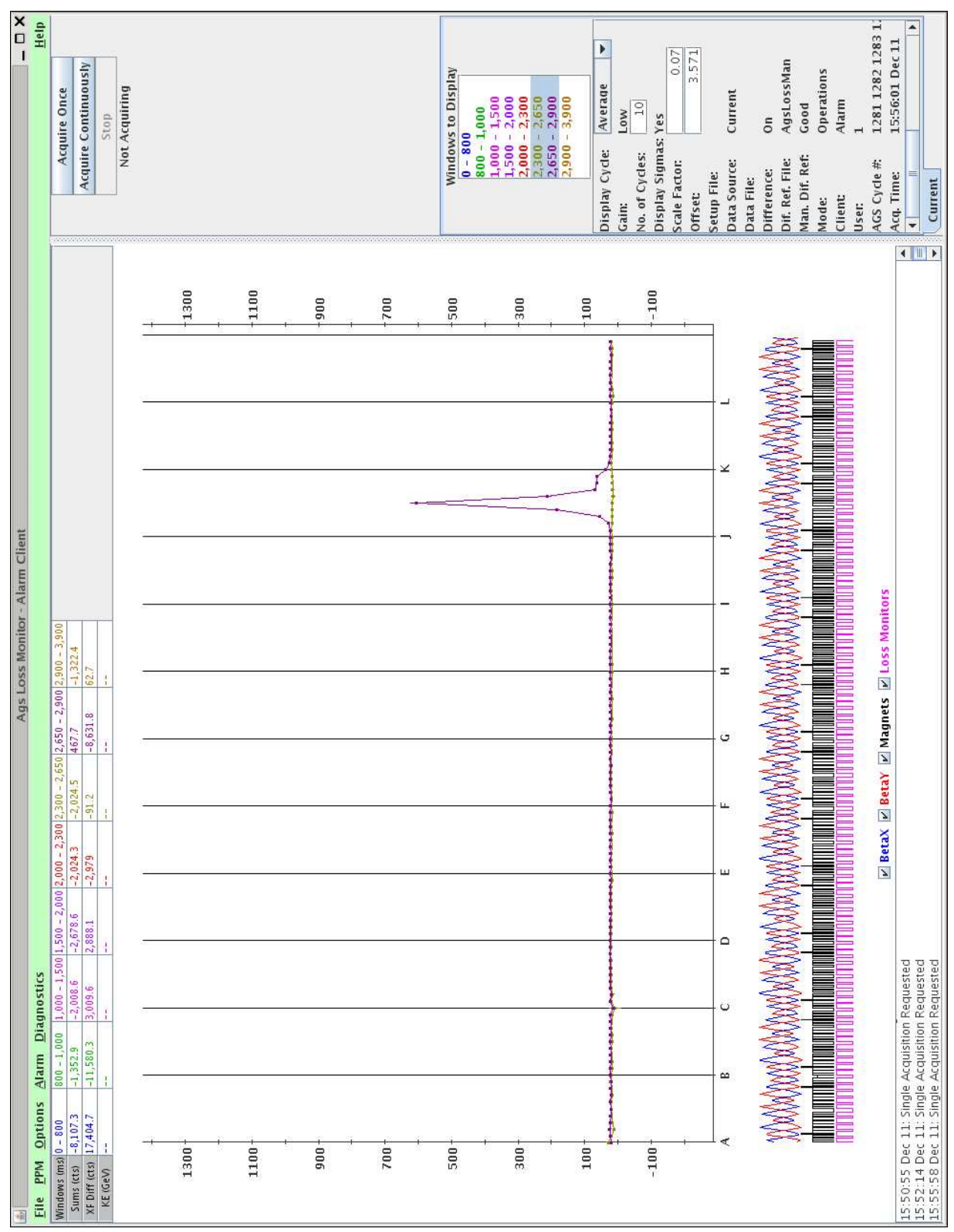

Figure 59: Loss pattern in AGS with some $3.5 \times 10^{9}$ gold ions being put into the J10 dump at extraction energy. Here the J7 stripper is being plunged into the circulating beam. 